\title{
A PROJECTIVE DESCRIPTION OF WEIGHTED INDUCTIVE LIMITS
}

BY

\author{
KLAUS D. BIERSTEDT, REINHOLD MEISE AND WILLIAM H. SUMMERS
}

\author{
Dedicated to Professor G. Köthe on the occasion of his 75 th birthday
}

\begin{abstract}
Considering countable locally convex inductive limits of weighted spaces of continuous functions, if $\mathfrak{V}=\left\{V_{n}\right\}_{n}$ is a decreasing sequence of systems of weights on a locally compact Hausdorff space $X$, we prove that the topology of $\mathfrak{V}_{0} C(X)=$ ind $_{n \rightarrow} C\left(V_{n}\right)_{0}(X)$ can always be described by an associated system $\bar{V}=\bar{V}_{\mathfrak{⿰}}$ of weights on $X$; the continuous seminorms on $\mathfrak{V}_{0} C(X)$ are characterized as weighted supremum norms. If $\mathfrak{V}=\left\{v_{n}\right\}_{n}$ is a sequence of continuous weights on $X$, a condition is derived in terms of $\mathfrak{V}$ which is both necessary and sufficient for the completeness (respectively, regularity) of the $(L B)$-space $\mathcal{V}_{0} C(X)$, and which is also equivalent to $\mathfrak{V}_{0} C(X)$ agreeing algebraically and topologically with the associated weighted space $C \bar{V}_{0}(X)$; for sequence spaces, this condition is the same as requiring that the corresponding echelon space be quasi-normable.

A number of consequences follow. As our main application, in the case of weighted inductive limits of holomorphic functions, we obtain, using purely functional analytic methods, a considerable extension of a theorem due to B. A. Taylor [37] which is useful in connection with analytically uniform spaces and convolution equations.

The projective description of weighted inductive limits also serves to improve upon existing tensor and slice product representations. Most of our work is in the context of spaces of scalar or Banach space valued functions, but, additionally, some results for spaces of functions with range in certain $(L B)$-spaces are mentioned.
\end{abstract}

Introduction. Countable locally convex inductive limits of weighted spaces of continuous or, in particular, holomorphic functions naturally arise in great profusion throughout such fields as linear partial differential equations and convolution equations, distribution theory and representation of distributions as boundary values of holomorphic functions, complex analysis in one and several variables, and spectral theory and the holomorphic functional calculus. However, perhaps because the structure of general locally convex inductive limits is rather intricate, functional analytic applications have been restricted by a widespread belief that it was not possible to describe an inductive limit topology or the corresponding continuous seminorms in a setting which is general enough to include a substantial part of the applications, while at the same time, and above all, being "useful" for providing convergence results and direct estimates in terms of the seminorms. One aim of the

Received by the editors March 23, 1981.

1980 Mathematics Subject Classification. Primary 46E10; 30H05, 42B99, 46A12, 46A14, 46A45, 46E40, 46M05, 46M40, 46N05. 
present article is to demonstrate that, in the case of weighted inductive limits, it is indeed possible (and, somewhat to our own surprise, not even too difficult) to give a natural, sufficiently general, and useful projective description of the limit space, the inductive limit topology, and the continuous seminorms.

In a setting which includes some of the more interesting cases, the fundamental problem to which we shall address ourselves in the sequel can be described roughly as follows: If $\mathfrak{V}=\left\{V_{n}\right\}_{n \in \mathbf{N}}$ is a decreasing sequence of systems $V_{n}$ of weights on a locally compact Hausdorff space $X, E$ is a locally convex space, and $A(X, E)$ is a linear subspace of the space $C(X, E)$ of all continuous $E$-valued functions on $X$, then is it possible to characterize the inductive limit topology of $\mathscr{V}_{0} A(X, E)=$ ind $_{n \rightarrow} A\left(V_{n}\right)_{0}(X, E)$ by a system of weighted seminorms? Or, in particular, when can $\mho_{0} A(X, E)$ be identified algebraically and topologically with its associated weighted space $A \bar{V}_{0}(X, E)$ (cf. [7])? Of course, our main interest lies in those cases where $A(X, E)=C(X, E)$ or when $A(X, E)$ is the space, say, of ( $E$-valued) holomorphic functions on an open subset $X$ of $\mathbf{C}^{N}(N \geqslant 1)$. Also, the case for scalar functions (i.e., when $E=C$ ) clearly deserves the bulk of our attention.

In $§ 0$, we make the notation precise, recall some preliminary facts (mainly from [7]), and discuss the basic problem in more detail. The general characterization results are contained in $\S 1$. First of all, we prove (Theorem 1.3) that, for a Banach space $E, C \bar{V}_{0}(X, E)$ always induces the inductive limit topology on its dense subspace $\mathfrak{V}_{0} C(X, E)$. It follows (Theorem 1.6) that $\mathfrak{V}_{0} A(X)=A \bar{V}_{0}(X)$ holds whenever $A(X)$ is a semi-Montel space under the compact-open topology and $\mathcal{V}=\left\{v_{n}\right\}_{n \in \mathbf{N}}$ is a decreasing sequence of strictly positive continuous weights satisfying the condition

(V) for each $n \in \mathrm{N}$, there exists $m>n$ such that $v_{m} / v_{n}$ vanishes at infinity.

Condition (V) is satisfied in virtually all applications, and Theorem 1.6, among other things, covers the case of holomorphic functions on an arbitrary open subset $X$ of $\mathbf{C}^{N}$, extending a theorem due to B. A. Taylor [37] in several respects.

Likewise in $\S 1$, we show, for instance, that the maximal system $\bar{V}$ of associated weights can be reduced to a smaller system of weights with a more manageable form, and we also deduce a number of refinements of the general theorems in particular cases. Finally, we note (Example 1.12) that a result analogous to Theorem 1.3, even in very restricted settings, does not hold for weighted spaces of type $C V(X)$; indeed, while $\mathcal{V} C(X)=C \bar{V}(X)$ is always valid algebraically, and the two spaces even have the same bounded subsets, the inductive limit topology can be strictly stronger than the weighted topology of the associated space $C \bar{V}(X)$.

In $\S 2$, we present a detailed study of the weighted $(L B)$-spaces $\mathfrak{V}_{0} C(X)$; i.e., the scalar case for a decreasing sequence $\mathscr{V}=\left\{v_{n}\right\}_{n \in \mathbf{N}}$ of strictly positive continuous weights on $X$. This includes a complete classification of several interesting properties in terms of the sequence $\mathcal{V}$. In particular, the algebraic equality $\mathcal{V}_{0} C(X)=C \bar{V}_{0}(X)$ is shown to hold (and hence the two spaces are topologically isomorphic) if, and only if, $\mathcal{V}$ is regularly decreasing, a condition which, by the way, coincides with the property $(\mathrm{wV})$ introduced in the appendix of [7]. Moreover, $\mathcal{V}$ being regularly decreasing is equivalent to $\mathcal{V}_{0} C(X)$ being $(\alpha-)$ regular, or boundedly retractive, or 
(sequentially) complete, respectively. Such a classification was apparently not even known in the case of sequence spaces (i.e., when $X=\mathbf{N}$ ) where $\mathscr{V}_{0} C(\mathbf{N})$ is an inductive limit of weighted $c_{0}$-spaces.

As one illustration of the utility of our theorems, we deduce consequences for $\varepsilon$-tensor products in $\S 3$, and thereby improve upon previous results from [7]. Among other things, we obtain a representation

$$
\Upsilon_{0} C(X, E)=\mathscr{V}_{0} C(X) \check{\otimes}_{\varepsilon} E
$$

for spaces of continuous vector valued functions (Theorem 3.1 and Corollary 3.3) and a slice product theorem

$$
\left(\mathscr{V}_{1} \otimes \mathscr{V}_{2}\right)_{0} C\left(X_{1} \times X_{2}\right)=\left(\mathscr{V}_{1}\right)_{0} C\left(X_{1}\right) \check{\otimes}_{\varepsilon}\left(\mathscr{V}_{2}\right)_{0} C\left(X_{2}\right)
$$

for continuous functions of several variables (Theorem 3.7) which had seemed inaccessible in [7] (without the projective characterization of weighted inductive limits). Again, some consequences for holomorphic functions (Corollaries 3.2 and 3.8) are derived.

$\$ 4$ is mainly devoted to a discussion of related work. We describe the relation between our Theorem 1.6 and Taylor's theorem [37] in some detail, as well as give reasons for the importance of such results in the theory of analytically uniform spaces (Ehrenpreis [11], Berenstein-Dostal [2]). In fact, our Theorem 1.6 has recently been applied by S. Hansen [18], [19] in his approach to the Fundamental Principle of Ehrenpreis, and by Grudzinski [17] in his study of convolution equations. We also point out that the spaces $\theta(\delta)$ in Ferrier's book [12] are covered by our Theorem 1.6 whereby the inductive limit topology of these spaces can be described in a natural way.

In the "concluding remarks" of $§ 5$, we sketch a (slightly) different approach which allows us to replace the local compactness hypothesis for $X$ in Theorem 1.6 by the condition that $X$ be completely regular and hemi-compact, thereby yielding consequences, say, for holomorphic functions on ( $D F M)$-spaces. Furthermore, for $(L B)$ spaces $E$ of various types, we briefly describe some results on the (strong) regularity of $\mathcal{V} C(X, E)$ and $\mathcal{V}_{0} C(X, E)$ which arise in connection with the associated spaces $C \bar{V}(X, E)$ and $C \bar{V}_{0}(X, E)$, respectively.

Historically speaking, a systematic investigation of weighted inductive limits was initiated in [7], and it is our purpose here to continue that work, although in a somewhat different spirit and from another point of view. A "lower estimate" or a "weighted hull" for the weighted inductive limit $\mathfrak{V}_{0} C(X, E)$ and its topology, an associated weighted space $C \bar{V}_{0}(X, E)$ in which the limit is continuously embedded, was constructed in [7], but it was used there only as a tool, while the fundamental problem of the exact relationship between these two spaces was only touched upon and had remained open. An important stimulus to undertaking our research was the theorem due to B. A. Taylor [37] which describes the topology of certain nuclear $(L B)$-spaces of entire functions. Though very useful for applications in the theory of analytically uniform spaces in the sense of Ehrenpreis [11], the result appeared somewhat isolated since Taylor's method of proof made extensive use of Hörmander's results and techniques in several complex variables. But, following an idea suggested 
by [7], we were able to construct a purely functional analytic proof of Taylor's theorem which has the advantage of being more general. (To do this, however, we make a "detour" through weighted spaces of continuous functions, where standard methods like partitions of unity are available, and then recover the result for holomorphic functions by means of an open mapping lemma due to Baernstein [1].) An analysis of the situation indicated that a general method was hidden behind our proof and, in fact, the previously constructed "weighted hull" $C \bar{V}_{0}(X, E)$ could give precise information about the inductive limit space $\mathcal{V}_{0} C(X, E)$ and its topology. From this, the present projective characterization of weighted inductive limits has evolved.

ACKNOWLEDGEMENT AND APOLOGY. This research project was started, more or less, during a visit by the last-named author at Universität-GesamthochschulePaderborn in the summer of 1977 , and we would like to express our appreciation for that support. Since that time, as each of us has reported on progress in one direction or another, the scope of the project has been repeatedly enlarged, but we think that the results have improved with the years. Nonetheless, we apologize for having taken so much time in bringing this paper to publication, and we thank several authors (Grudzinski, Hansen, Hollstein, and Schmets, among others) for their patience with the long write-up procedure of an article which has already been quoted (and, sometimes, unfortunately, also misquoted) several times.

0. Preliminaries; statement of the problem. The notation, definitions, and conventions introduced below will be used throughout the sequel (sometimes without further reference).

0.1 . Weighted spaces. Let $X$ denote a completely regular Hausdorff space. A nonnegative real valued function on $X$ which is upper semicontinuous (u.s.c.) will be called a weight (on $X$ ). If $U$ and $V$ are two sets of weights on $X$, we write $U \leqslant V$ whenever, corresponding to each $u \in U$, there exist $v \in V$ and $\lambda>0$ such that $u \leqslant \lambda v$ (pointwise on $X$ ). If, given any $x \in X$, there is some $v \in V$ for which $v(x)>0$, we will write $V>0$. A set $V$ of weights on $X$ is said to be directed upward provided that, for every pair $v_{1}, v_{2} \in V$ and each $\lambda>0$, it is possible to find $v \in V$ so that $\lambda v_{1} \leqslant v$ and $\lambda v_{2} \leqslant v$. Since there will be no loss of generality, we shall hereafter assume that sets of weights are directed upward. If a set $V$ of weights on $X$ also satisfies $V>0$, then $V$ will be referred to as a system of weights (or a Nachbin family) on $X$. The system $\mathscr{K}=\mathscr{K}(X)=\left\{\lambda 1_{K} ; \lambda>0, K \subset X, K\right.$ compact $\}$, where $1_{K}$ denotes the characteristic function of the set $K$, will be helpful to us in what follows.

We will let $E$ denote a (Hausdorff) locally convex (1.c.) topological vector space which, because we wish to treat also the case of holomorphic functions, is assumed to be complex. The set of all continuous seminorms on $E$ will be denoted by $\operatorname{cs}(E)$, while we will write $C(X, E)$ to indicate the collection of all continuous functions mapping $X$ into $E$. We next pause briefly to recall that a real (or complex) valued function $g$ defined on $X$ is said to vanish at infinity if, corresponding to each $\varepsilon>0$, there exists a compact subset $K$ of $X$ such that $x \in X \backslash K$ implies $|g(x)| \leqslant \varepsilon$. Now, taking a system $V$ of weights on $X$ and setting

$$
q_{v, p}(f)=\sup \{v(x) p(f(x)) ; x \in X\}
$$


where $v \in V, p \in \operatorname{cs}(E)$, and $f \in C(X, E)$, we put

$$
C V(X, E)=\left\{f \in C(X, E) ; q_{v, p}(f)<\infty \text { for all } v \in V \text { and all } p \in c s(E)\right\}
$$

and

$$
\begin{aligned}
& C V_{0}(X, E)=\{f \in C(X, E) ; v(p \circ f) \text { vanishes at infinity } \\
& \text { for all } v \in V \text { and all } p \in c s(E)\} .
\end{aligned}
$$

If $v \in V$ and $p \in c s(E)$, then $q_{v, p}$ can be regarded as a seminorm on either of the complex vector spaces $C V_{0}(X, E)$ or $C V(X, E)$; we assume that both of these spaces are equipped with the Hausdorff locally convex topology induced by $\left\{q_{v, p} ; v \in V\right.$, $p \in \operatorname{cs}(E)\}$. In particular, the space $C \mathcal{K}(X, E)$ has the topology of uniform convergence on the compact subsets of $X$, which will hereafter be denoted by co.

More generally, if $V$ is a system of weights on $X$ and $A(X, E)$ is some predetermined linear subspace of $C(X, E)$, we put $A V(X, E)=A(X, E) \cap C V(X, E)$ and $A V_{0}(X, E)=A(X, E) \cap C V_{0}(X, E)$. Then, taking $A(X, E)=C(X, E)$, for instance, we have $A V(X, E)=C V(X, E)$ and $A V_{0}(X, E)=C V_{0}(X, E)$. Or, if $X$ happens to be either an open subset of $\mathbf{C}^{N}, N \geqslant 1$, or any other locally convex space, a complex analytic manifold, or a complex space, we can take $A(X, E)=H(X, E)$, the space of all holomorphic (i.e., Gâteaux-analytic and continuous) functions mapping $X$ into $E$. While this may be the most important case in view of the applications we have in mind, other examples include the harmonic functions or solutions of certain homogeneous linear hypoelliptic partial differential equations (if $E=\mathbf{C}$ ), as well as the sheaves of "F्F-morphic" functions considered in [8].

In the special case that $V=\{\lambda v ; \lambda>0\}$ for a single weight $v$ on $X$, we will write $A v(X, E)$ and $A v_{0}(X, E)$ instead of $A V(X, E)$ and $A V_{0}(X, E)$, respectively. (Of course, since we are requiring that $V>0$, this requires that $v(x)>0$ for all $x \in X$.) In case $E=\mathrm{C}$, we will omit $E$ from our notation and write $A V(X), A V_{0}(X), A v(X)$, and $A v_{0}(X)$; if $v$ is a weight on $X$, we then put $q_{v}=q_{v, p}$ where $p(z)=|z|, z \in \mathbf{C}$. Similarly, if $E=(E,\|\cdot\|)$ is a normed space, we will simply write $q_{v}$ instead of $q_{v,\|\cdot\| \cdot}$

0.2. Inductive limits of weighted spaces and the associated weighted hull. For each $n \in \mathbf{N}$, let $V_{n}$ denote a system of weights on $X$, and assume that $V_{n+1} \leqslant V_{n}$, $n=1,2, \ldots$ Denoting the sequence $\left\{V_{n}\right\}_{n \in \mathrm{N}}$ by $\mathcal{V}$, we define two associated systems of weights on $X$ :

(i) If $v_{n} \in V_{n}$ and $\alpha_{n}>0, n=1,2, \ldots$, then putting $v(x)=\inf \left\{\alpha_{n} v_{n}(x), n \in \mathbf{N}\right\}$, $x \in X$, clearly defines a weight on $X$. The set $\check{V}_{\mathfrak{V}}$ consisting of all such pointwise infima is, in turn, a system of weights on $X$.

(ii) All those weights on $X$ which are pointwise majorized by some member of $\check{V}_{q}$ also form a system which we denote by $\bar{V}_{\mathfrak{V}}$ and term the maximal system associated with $\mathcal{T}$.

Obviously, $\bar{V}_{\mathscr{V}} \leqslant \check{V}_{\mathfrak{V}}$ and $\check{V}_{\mathfrak{V}} \leqslant \bar{V}_{\mathfrak{V}}$, while $\bar{V}_{\mathfrak{V}}$ contains every system $V$ of weights on $X$ with the property that $V \leqslant V_{n}$ for every $n \in \mathbf{N}$. So, for instance, if we require $\mathcal{K}(X) \leqslant V_{n}$ for each $n \in \mathbf{N}$, then $\mathscr{K}(X) \subset \bar{V}_{\mathscr{V}}$. Attention will sometimes be restricted to the set $\tilde{V}_{\mathscr{V}}$ consisting of all continuous elements in $\bar{V}_{\mathfrak{v}}$. If $\tilde{V}_{\Im}>0$, then it is a system of weights on $X$. 
In the special case that $V_{n}=\left\{\lambda v_{n} ; \lambda>0\right\}, n \in \mathbf{N}$, we will also denote the sequence $\left\{v_{n}\right\}_{n \in \mathrm{N}}$ by $\mathcal{V}$ for simplicity; observe that $v \in \bar{V}_{\mathfrak{V}}$ if, and only if, $v$ is a weight on $X$ and $v / v_{n}$ is bounded for $n=1,2, \ldots$ If the sequence $\mathcal{V}=\left\{V_{n}\right\}_{n}$ or $\left\{v_{n}\right\}_{n}$ is clear from context, we will omit $\mathcal{V}$ from our notation and simply write $\mathscr{V}, \bar{V}$, and $\tilde{V}$.

Proposition. Let $X$ denote a locally compact and $\sigma$-compact space and $\mathscr{V}=\left\{v_{n}\right\}_{n \in \mathbf{N}}$ a decreasing sequence of strictly positive continuous weights $v_{n}$ on $X$. Then the set $\tilde{V}$ is equivalent to $\bar{V}$; i.e., not only is $\tilde{V} \subset \bar{V}$, but also $\bar{V} \leqslant \tilde{V}$ (whence, clearly, $A \bar{V}(X, E)=$ $A \tilde{V}(X, E)$ and $A \bar{V}_{0}(X, E)=A \tilde{V}_{0}(X, E)$ algebraically and topologically).

Proof. It suffices to show that an arbitrary $v=\inf \left\{\alpha_{n} v_{n} ; n \in \mathbf{N}\right\} \in \check{V}$ is dominated pointwise by a continuous weight $\bar{v} \in \check{V}$. But, fixing a sequence $\left(K_{m}\right)_{m \in \mathrm{N}}$ of compact subsets of $X$ with $K_{m} \subset \stackrel{\circ}{K}_{m+1}, m=1,2, \ldots$, and $X=\cup_{m \in N} K_{m}$, we can inductively choose positive numbers $\beta_{n} \geqslant \alpha_{n}, n=1,2, \ldots$, such that $\beta_{1}=\alpha_{1}$ and

$$
\begin{array}{r}
\beta_{n}\left(\inf \left\{v_{n}(x) ; x \in K_{n}\right\}\right) \geqslant \sup \left\{\beta_{j} v_{j}(x) ; x \in K_{n-1}, j=1, \ldots, n-1\right\}, \\
n=2,3, \ldots
\end{array}
$$

Then $\bar{v}=\inf \left\{\beta_{n} v_{n} ; n \in \mathbf{N}\right\} \in \check{V}$ and obviously $v \leqslant \bar{v}$. To see that $\bar{v}$ is continuous (and hence belongs to $\tilde{V}$ ), fix $m \in \mathbf{N}$ and note that, for $n=m+1, m+2, \ldots$,

$$
\begin{aligned}
\inf \left\{\beta_{n} v_{n}(x) ; x \in K_{m}\right\} & \geqslant \beta_{n} \inf \left\{v_{n}(x) ; x \in K_{n}\right\} \\
& \geqslant \sup \left\{\beta_{j} v_{j}(x) ; x \in K_{n-1}, j=1, \ldots, n-1\right\} \\
& \geqslant \sup \left\{\beta_{j} v_{j}(x) ; x \in K_{m}, j=1, \ldots, n-1\right\} ;
\end{aligned}
$$

for each $y \in K_{m}$, therefore,

$$
\begin{aligned}
\beta_{n} v_{n}(y) & \geqslant \inf \left\{\beta_{n} v_{n}(x) ; x \in K_{m}\right\} \\
& \geqslant \sup \left\{\beta_{j} v_{j}(x) ; x \in K_{m}, j=1, \ldots, n-1\right\} \geqslant \beta_{m} v_{m}(y) .
\end{aligned}
$$

Thus $\bar{v}=\inf \left\{\beta_{n} v_{n} ; n=1, \ldots, m\right\}$ pointwise on $K_{m}$, and, since all the weights $v_{n}$ are continuous, $\bar{v}$ is continuous on $K_{m}$. Because $m \in \mathbf{N}$ was arbitrary, this concludes the proof.

For a l.c. space $E$, a sequence $\mathfrak{V}=\left\{V_{n}\right\}_{n \in \mathbf{N}}$ of systems of weights on $X$ with $V_{n+1} \leqslant V_{n}, n=1,2, \ldots$, and a subspace $A(X, E)$ of $C(X, E)$, we consider the following (weighted) l.c. inductive limits:

$$
\checkmark A(X, E)=\operatorname{ind}_{n \rightarrow} A V_{n}(X, E) \text {, respectively, } \mho_{0} A(X, E)=\operatorname{ind}_{n \rightarrow} A\left(V_{n}\right)_{0}(X, E) ;
$$

i.e., we take the (increasing) union of all spaces $A V_{n}(X, E)$, respectively, $A\left(V_{n}\right)_{0}(X, E)$, and endow it with the finest (here always Hausdorff) 1.c. topology which makes all the injections $A V_{n}(X, E) \rightarrow \mathscr{V} A(X, E)$, respectively, $A\left(V_{n}\right)_{0}(X, E)$ $\rightarrow \Upsilon_{0} A(X, E)$, continuous. It is easy to see that $\Upsilon_{A} A(X, E)$ can be continuously injected into $A \bar{V}(X, E)$, as can $\mathcal{V}_{0} A(X, E)$ into $A \bar{V}_{0}(X, E)$. The investigation set forth in this article centers around the further relationships between these two pairs of spaces. 
Let us now pause to observe that, according to our conventions, choosing $A(X, E)=C(X, E)$ defines $\mho_{C}(X, E)$ and $\mho_{0} C(X, E)$, while $\mho_{H}(X, E)$ and $\mathfrak{\mho}_{0} H(X, E)$ arise by putting $A(X, E)=H(X, E)$. Also, $\Upsilon_{A} A(X)$ and $\mathfrak{V}_{0} A(X)$ stand for $\mathfrak{V}_{A}(X, \mathrm{C})$ and $\mathfrak{\mho}_{0} A(X, \mathrm{C})$, respectively.

Assume next that $\left\{E_{n}, i_{n m}\right\}_{n, m \in \mathbf{N}}$ is an injective system of 1.c. spaces with the 1.c. inductive limit $E=\operatorname{ind}_{n \rightarrow} E_{n}$ being a Hausdorff space. We will also consider "diagonal" inductive limits of the form

$$
\mathcal{Q} \mathcal{V} A(X, E)=\operatorname{ind}_{n \rightarrow} A V_{n}\left(X, E_{n}\right) \text { and } \mathscr{D} \mathbb{V}_{0} A(X, E)=\operatorname{ind}_{n \rightarrow} A\left(V_{n}\right)_{0}\left(X, E_{n}\right)
$$

(cf. [7]).

0.3. Strong regularity conditions for l.c. inductive limits. Let us recall some notation introduced in [6]. If the 1.c. space $E$ is represented as the l.c. inductive limit ind ${ }_{n \rightarrow} E_{n}$ of an increasing sequence of 1.c. spaces $E_{n} \subset E$ (each endowed with a topology which is stronger than the one induced by $E$ ), the inductive system $\left(E_{n}\right)_{n \in \mathbf{N}}$ (or its limit space $E$ ) is called

(i) regular (respectively, compactly regular) if, for every bounded (respectively, compact) subset $B$ of $E$, there exists $n \in \mathbf{N}$ such that $B$ is a bounded (respectively, compact) subset of $E_{n}$;

(ii) boundedly retractive if, for every bounded subset $B$ of $E$, there exists $n \in \mathbf{N}$ such that $B$ is bounded in $E_{n}$ and the topologies of $E$ and $E_{n}$ coincide on $B$;

(iii) strongly boundedly retractive if $E$ is regular and, for every $n \in \mathbf{N}$, there exists $m \geqslant n$ such that the topologies of $E$ and $E_{m}$ coincide on each bounded subset $B$ of $E_{n}$.

It is clear that

strongly boundedly retractive $\Rightarrow$ boundedly retractive $\Rightarrow$ compactly regular, and one can also show (under our countability assumption) that

$$
\text { compactly regular } \Rightarrow \text { regular } \Rightarrow \text { Hausdorff. }
$$

Boundedly retractive inductive limits preserve quasi-completeness. On the other hand, Neus [25] proved that a compactly regular inductive limit $E=$ ind $_{n \rightarrow} E_{n}$ of normed spaces $E_{n}$ is already boundedly retractive whence clearly also strongly boundedly retractive. It then follows that a compactly regular inductive limit of a sequence of Banach spaces is a quasi-complete $(D F)$-space and hence complete. In particular, every countable strict inductive limit $E=\operatorname{ind}_{n \rightarrow} E_{n}$ where $E_{n}$ is closed in $E_{n+1}$ for $n=1,2, \ldots$, and each Silva space (i.e., countable injective compact 1.c. inductive limit of Banach spaces) is strongly boundedly retractive.

Finally, using a theorem of Grothendieck on countable 1.c. inductive limits of (DF)-spaces (cf. [23, I, §29, 5.(4)]), if the 1.c. space $E=\operatorname{ind}_{n \rightarrow} E_{n}$ is an inductive limit of complete $(D F)$-spaces $E_{n}$, then the second requirement in the definition of the strong boundedly retractive property (i.e., for every $n \in \mathbf{N}$, there exists $m \geqslant n$ such that the topologies of $E$ and $E_{m}$ coincide on each bounded subset $B$ of $E_{n}$ ) already suffices to guarantee regularity. (This is a remark due to J. Mujica.)

0.4. Condition (V) and its consequences. At this point, we would like to recall some results from [7] which will be needed later. 
The following is clearly a sufficient condition (in terms of $X$ and $\mathfrak{V}$ ) for $\mathfrak{V} A(X, E)=\mathscr{V}_{0} A(X, E)$ :

(V) For each $n \in \mathbf{N}$ there exists $m>n$ such that, given an arbitrary element $v_{m} \in V_{m}$, some $v_{n} \in V_{n}$ can be found so that, for every $\varepsilon>0$, there exists $K \subset X, K$ compact, with $v_{m}(x) \leqslant \varepsilon v_{n}(x)$ for all $x \in X \backslash K$.

In the special case that $V_{n}=\left\{\lambda v_{n} ; \lambda>0\right\}, n=1,2, \ldots$, condition (V) can be expressed as follows:

(V) For every $n \in \mathbf{N}$ there exists $m>n$ so that $v_{m} / v_{n}$ vanishes at infinity.

Further, if $\inf \left\{v_{n}(x) ; x \in K\right\}>0$ for each compact subset $K$ of $X$ and each $n=1,2, \ldots$, then, in the presence of condition (V), a weight $v$ on $X$ belongs to $\bar{V}$ if, and only if, $v / v_{n}$ vanishes at infinity for each $n \in \mathbf{N}$.

Observe that a completely regular space $X$ on which there is a decreasing sequence $\mathcal{V}=\left\{v_{n}\right\}_{n \in \mathrm{N}}$ of (strictly positive) weights with property (V) must necessarily be $\sigma$-compact, and hence countable at infinity in the locally compact case; for $n \in \mathbf{N}$ and $m \geqslant n$ as in condition (V), $X$ is the union of the compact sets

$$
\left\{x \in X ; \frac{v_{m}(x)}{v_{n}(x)} \geqslant \frac{1}{k}\right\}, \quad k=1,2, \ldots
$$

Thus, whenever $X$ is locally compact and there exists a sequence $\mathscr{V}=\left\{v_{n}\right\}_{n}$ on $X$ which satisfies $(\mathrm{V}),(C(X), \mathrm{co})$ is complete and metrizable whereby any semi-Montel subspace $(A(X), \mathrm{co})$ of $(C(X), \mathrm{co})$, being quasi-complete, is already complete and hence closed in $(C(X), \mathrm{co})$.

We turn to other consequences of condition (V).

(a) Let us suppose that $\mathscr{K} \leqslant V_{n}$ for each $n \in \mathbf{N}$ and that $\mathfrak{V}=\left\{V_{n}\right\}_{n}$ satisfies (V). If we fix $n$ and $m$ as in (V), and let $B$ denote an arbitrary bounded subset of $A V_{n}(X, E)$, then $A V_{m}(X, E), \mathcal{T} A(X, E)=\mathscr{V}_{0} A(X, E)$, and the compact-open topology all induce the same topology on $B$.

(b) If, in addition, $A V_{n}(X, E)$ is a $(D F)$-space for each $n \in \mathbf{N}$, the inductive limit $\checkmark A(X, E)=\operatorname{ind}_{n \rightarrow} A V_{n}(X, E)$ is strongly boundedly retractive.

(c) Under the conditions of (b), let us also assume that $X$ is a $k_{\mathbf{R}^{-s p a c e}}$ and $E$ is complete. Then $\mathscr{V} A(X, E)$ is also complete whenever $A(X, E)$ is closed in $(C(X, E), \mathrm{co})$.

Instead of $(\mathrm{V})$, a weaker condition $(\mathrm{wV})$, which is recalled in $\$ 2$ below, proved sufficient for (b) and (c) (see the appendix of [7]), but (wV) does not imply $\mathcal{V} A(X, E)=\mathcal{V}_{0} A(X, E)$ or the topological part of (a), nor does it give the next result.

(d) Let $\mathcal{V}=\left\{v_{n}\right\}_{n \in \mathbf{N}}$ denote a decreasing sequence of weights $v_{n}$ on $X$ such that $\inf \left\{v_{n}(x) ; x \in K\right\}>0$ for each compact subset $K$ of $X$ and $n=1,2, \ldots$ If we suppose that condition (V) holds and that $(A(X), \mathrm{co})$ is a semi-Montel space, then $\mathscr{V} A(X)=\Upsilon_{0} A(X)$ is a Silva (or (DFS)-) space, and hence is separable, complete, Montel, and the strong dual of a Fréchet-Schwartz space. Moreover, in this case, $\mathfrak{V} A(X)=\mathfrak{V}_{0} A(X)$ is a topological linear subspace of $\mathscr{V} C(X)=\mathfrak{V}_{0} C(X)$.

The last statement in (d) is the key to our results in the context of spaces of holomorphic functions (cf. $\$ 4$ for a discussion of applications); it allows us, in particular, to deduce Theorem 1.6 below from a corresponding result for spaces of 
continuous functions (Theorem 1.3) in the proof of which we utilize functions with compact support and continuous partitions of unity. Thus, even though this assertion has already been established in [7, Satz 1.14], we shall here try to convey some insight into the argument.

Our main tool is an open mapping lemma due to A. Baernstein II [1] which asserts that a continuous linear mapping $T$ from a semi-Montel space $F$ into a $(D F)$-space $E$ with the property that the preimage $T^{-1}(B)$ of each bounded subset $B$ in $E$ is bounded in $F$ must in fact already be a topological isomorphism into $E$. (Baernstein uses some duality theory to establish his lemma; the essential point lies in an application of Ptak's open mapping theorem to the transpose of $T$ with respect to the strong duals. Different proofs and some extensions have subsequently been given by B. Ernst and W. Ruess.)

To obtain the final statement from (d) above, it is then enough to apply Baernstein's lemma to the inclusion mapping $T: \mathcal{V} A(X) \rightarrow \mathscr{V} C(X)$ while taking into account the first part of (d) along with the fact that $\mathcal{V} C(X)$ is always a regular inductive limit in our setting.

Moreover, this final assertion in (d) suggests the following (rather general)

Problem. Under what conditions does either $\mathfrak{V} A(X, E)$ or $\mathcal{V}_{0} A(X, E)$ become a topological linear subspace of $\mathcal{V} C(X, E)$ or $\mathcal{V}_{0} C(X, E)$, respectively?

This special case of the general "subspace problem" for inductive limits is of particular interest for $A(X, E)=H(X, E)$ (cf. [7]). A positive solution is given in (d) only under restrictive conditions; in fact, we do not know whether such a result is also true, say, for holomorphic functions with values in a Banach space, and it is not clear whether the inductive limit topology of $\mathfrak{V}_{0} A(X)$ is in general strictly stronger than the topology induced by $\mathcal{V}_{0} C(X)$.

In order to clarify the role of condition (V), it may be useful to note a partial converse to (a) in a restricted setting.

Proposition. Let $X$ be a completely regular space and let $\mathfrak{V}=\left\{v_{n}\right\}_{n}$ denote a decreasing sequence of strictly positive continuous weights on $X$. For fixed $n \in \mathbf{N}$ and $m>n$, if $C v_{m}(X)$ induces co on each bounded subset $B$ of $C v_{n}(X)$, then the quotient $v_{m} / v_{n}$ must necessarily vanish at infinity.

Proof. Supposing that $v_{m} / v_{n}$ does not vanish at infinity, there exists $\varepsilon>0$ such that $\sup \left\{v_{m}(x) / v_{n}(x) ; x \in X \backslash K\right\}>\varepsilon$ for each compact subset $K$ of $X$. Now, let $\bigodot$ denote the system of all compact subsets of $X$, directed with respect to inclusion. To each $K \in \mathcal{C}$, we choose a point $x_{K} \in X \backslash K$ with $v_{m}\left(x_{K}\right) / v_{n}\left(x_{K}\right)>\varepsilon$. Since $X$ is completely regular, there exists a continuous function $f_{K}$ on $X$ with $0 \leqslant f_{K} \leqslant 1$, $f_{K}\left(x_{K}\right)=1$, and $\left.f_{K}\right|_{K} \equiv 0, K \in \mathcal{C}$.

Putting $g_{K}=f_{K} / v_{n}$, we observe that $\left\{g_{K} ; K \in \mathcal{C}\right\}$ is a bounded net in $C v_{n}(X)$ which clearly converges to zero with respect to co but fails to converge to zero in $C v_{m}(X)$ because

$$
\begin{aligned}
q_{v_{m}}\left(g_{K}\right) & =\sup \left\{v_{m}(x)\left|g_{K}(x)\right| ; x \in X\right\} \\
& \geqslant v_{m}\left(x_{K}\right)\left|g_{K}\left(x_{K}\right)\right|=\frac{v_{m}\left(x_{K}\right)}{v_{n}\left(x_{K}\right)}>\varepsilon, \quad K \in \mathcal{C} .
\end{aligned}
$$


0.5. The basic problem. Given a completely regular space $X$, a sequence $\mathfrak{V}=$ $\left\{V_{n}\right\}_{n \in \mathrm{N}}$ of systems of weights on $X$ with $V_{n+1} \leqslant V_{n}, n=1,2, \ldots$, a l.c. space $E$, and a subspace $A(X, E)$ of $C(X, E)$, the basic problem is to determine when

$$
\mathcal{V} A(X, E)=A \bar{V}(X, E) \text { and } \mathcal{V}_{0} A(X, E)=A \bar{V}_{0}(X, E)
$$

algebraically or both algebraically and topologically. If all the systems $V_{n}$ consist of continuous functions, one may also be interested in replacing the maximal system $\bar{V}$ assciated with $\mathfrak{V}$ by $\tilde{V}$ or, more generally, by smaller subsystems of weights which have additional properties.

As a superficial inspection of the situation and some relevant examples tend to show, the problem should not be considered as a question of whether the two identities hold in each case. Instead, it consists in finding (rather) general conditions on $X, \mathcal{V}, E$, and $A(X, E)$ which imply equality and which are satisfied in concrete examples important in the applications. (In fact, this problem first arose in these much more restricted settings.) After first establishing the general results, we will discuss examples and applications (in $\$ \S 3$ and 4) in order to illustrate our methods.

An interpretation of an algebraic equality, say $\mathscr{T} C(X, E)=C \bar{V}(X, E)$, might be that $\bar{V}$ yields a complete description of the weight conditions which the functions in the inductive limit space satisfy, while the space $C \bar{V}(X, E)$, on the other hand, is exhausted by the increasing sequence $\left(C V_{n}(X, E)\right)_{n \in \mathrm{N}}$ of subspaces. However, if $A \bar{V}_{0}(X, E)$ induces the inductive limit topology on its subspace $\mathcal{V}_{0} A(X, E)$, then this fact is much more interesting because it yields a ready, and in some sense the best possible, description of the continuous seminorms for the otherwise quite abstract inductive limit topology; the natural topology of each space $A\left(V_{n}\right)_{0}(X, E), n=$ $1,2, \ldots$, is given by a system of weighted seminorms, but it is not at all clear, $a$ priori, that the inductive limit topology of $\mathcal{V}_{0} A(X, E)$ can be characterized in the same way. Of course, an algebraic and topological identity $\mathcal{V}_{0} A(X, E)=A \bar{V}_{0}(X, E)$ also tells us something about the weighted topology of $A \bar{V}_{0}(X, E)$.

It should perhaps be further emphasized that a major difficulty which has to be overcome when dealing with the (natural) 1.c. inductive limit topologies occurring in many parts of analysis is the fact that in general there exists no nontrivial description of the continuous seminorms in such a topology: But yet, direct estimates and computations require a useful characterization of such seminorms! In our special situation, much information on the weighted spaces $A \bar{V}_{0}(X, E)$ is already available, and therefore, in the presence of a complete identity $\mathfrak{V}_{0} A(X, E)=$ $A \bar{V}_{0}(X, E)$, the interplay between the two structures can be used to resolve a number of questions concerning these spaces.

It is quite natural to split our original problem into two different parts: a scalar one $(E=\mathbf{C})$ or, as it turns out, the case of Banach range spaces $E$, where most of the methods developed in the scalar case still work, and a second in which more general 1.c. spaces $E$ are admitted. Given a solution of the first part of our problem, the connection of the second part with questions on $\varepsilon$-products and $\varepsilon$-tensor products (terms defined in $\$ 3$ below) becomes clear. Since, for example, $A\left(V_{n}\right)_{0}(X, E)=$ $A\left(V_{n}\right)_{0}(X) \varepsilon E, n=1,2, \ldots$, and $A \bar{V}_{0}(X, E)=A \bar{V}_{0}(X) \varepsilon E$ hold under fairly general 
hypotheses on $X, \mathcal{T}, E$, and $A(X, E)$, we see the relation to the abstract problem of knowing when inductive limits and $\varepsilon$-products commute, and some progress has already been realized in this direction in [7]. Results in general topological tensor product theory (see [16]) then suggest trying countable inductive limits $E=$ ind $_{n \rightarrow} E_{n}$ of Banach spaces as a first generalization and to take ( $D F$ )-type spaces $E$ as the next step.

In this paper, we will primarily concentrate on the case of normed spaces $E$. However, the reader will find some results for more general 1.c. range spaces $E$ in $\S \S 3$ and 5. A study of criteria for the commutativity of inductive limits and $\varepsilon$-tensor products has recently been undertaken in [20] where, using the results of a previous version of this article, Hollstein obtains new solutions in some (restricted) vector-valued cases, mainly when $A(X, E)=C(X, E)$ (see also 3.3 below).

1. The general results. Throughout this section, we use the conventions introduced in 0.1 and 0.2 , but two additional hypotheses will be in effect. First, we assume that $X$ is also locally compact. This being the case, it is clear that, given any system $V$ of weights on $X$, the space $C_{c}(X, E)$ of all $E$-valued continuous functions on $X$ which have compact support is densely contained in $C V_{0}(X, E)$. We will further require that $E$ is a normed space.

Before we prove our main result on the relation between $\mathcal{V}_{0} C(X, E)$ and $C \bar{V}_{0}(X, E)$, we need some preparation; the essential idea is contained in the following lemma.

1.1. LemMa. $\mathcal{V}_{0} C(X, E)$ and $C \bar{V}_{0}(X, E)$ induce the same topology on the dense subspace $C_{c}(X, E)$.

Proof. The canonical injection of $\mathfrak{V}_{0} C(X, E)$ into $C \bar{V}_{0}(X, E)$ being continuous, it suffices to show that, given an arbitrary neighborhood $U$ of zero in $\mathcal{V}_{0} C(X, E)$, there exists $\bar{v} \in \bar{V}$ such that $\left\{f \in C_{c}(X, E) ; q_{v}(f)=\sup _{x \in X} \bar{v}(x)\|f(x)\|<1\right\}$ is contained in $U$. Now, since $U$ is a neighborhood of zero in the l.c. inductive limit topology and each $V_{n}$ is directed upward, there exist $v_{n} \in V_{n}, n=1,2, \ldots$, such that $\Gamma\left(\cup_{n \in \mathrm{N}} B_{n}\right) \subset U$ where $B_{n}=\left\{f \in C\left(V_{n}\right)_{0}(X, E) ; q_{v_{n}}(f) \leqslant 1\right\}$ and $\Gamma$ denotes the absolutely convex hull. Putting $\bar{v}=\inf \left\{2^{n} v_{n} ; n \in \mathbf{N}\right\}$, we have $\bar{v} \in \check{V} \subset \bar{V}$; fix $f \in C_{c}(X, E)$ with $q_{v}(f)<1$. For each $n \in \mathbf{N}$,

$$
F_{n}=\left\{x \in X ; 2^{n} v_{n}(x)\|f(x)\| \geqslant 1\right\}
$$

is a closed subset of $\operatorname{supp} f$. If $x \in \bigcap_{n \in \mathrm{N}} F_{n}$, then $2^{n} v_{n}(x)\|f(x)\| \geqslant 1$ for all $n \in \mathbf{N}$ whereby $\bar{v}(x)\|f(x)\| \geqslant 1$. But this contradicts $q_{v}(f)<1$, and so $\bigcap_{n \in N} F_{n}$ is necessarily void.

Therefore, setting $U_{n}=X \backslash F_{n}, n \in \mathbf{N}$, since $\operatorname{supp} f$ is compact, there exists $m \in \mathbf{N}$ so that $\operatorname{supp} f \subset \cup_{n=1}^{m} U_{n}$. Now let $\left\{\varphi_{n}\right\}_{n=1}^{m} \subset C_{c}(X)$ be a finite continuous partition of unity on supp $f$ which is subordinate to the covering $\left\{U_{n}\right\}_{n=1}^{m}$. Putting $g_{n}=2^{n} \varphi_{n} f, 1 \leqslant n \leqslant m$, we note that $g_{n} \in C_{c}(X, E) \subset C\left(V_{n}\right)_{0}(X, E)$, while $g_{n}(x)=$ 0 if $x \in X \backslash U_{n}=F_{n}$ and $x \in U_{n}$ implies

$$
v_{n}(x)\left\|g_{n}(x)\right\|=\varphi_{n}(x) 2^{n} v_{n}(x)\|f(x)\|<1 ;
$$


i.e., $g_{n} \in B_{n}, 1 \leqslant n \leqslant m$. Consequently,

$$
f=\sum_{n=1}^{m} \varphi_{n} f=\sum_{n=1}^{m} 2^{-n} g_{n} \in \Gamma\left(\bigcup_{n \in \mathbf{N}} B_{n}\right) \text {. }
$$

1.2. LEMMA. Given a l.c. space $\left(E_{1}, \tau_{1}\right)$, let $E_{2}$ denote a linear subspace and $\tau_{2}$ a l.c. topology on $E_{2}$ which is finer than the topology induced by $\tau_{1}$. If $\tau_{1}$ and $\tau_{2}$ induce the same topology on some dense linear subspace $D$ of $\left(E_{2}, \tau_{2}\right)$, then $\tau_{2}=\tau_{1 \mid E_{2}}$.

Proof. Since $\tau_{1 \mid D}=\tau_{2 \mid D}$, for any closed neighborhood of zero $U$ in $\left(E_{2}, \tau_{2}\right)$ we can find an open neighborhood of zero $V$ in $\left(E_{1}, \tau_{1}\right)$ such that $V \cap D \subset U \cap D$. Now, since $D$ is dense in $\left(E_{2}, \tau_{2}\right)$,

$$
V \cap E_{2} \subseteq \overline{V \cap E_{2}}=\overline{V \cap D} \subset \bar{U}=U,
$$

where all closures are taken in $\left(E_{2}, \tau_{2}\right)$. Hence we have also $\tau_{1 \mid E_{2}} \geqslant \tau_{2}$.

The inductive limit topology on $\Upsilon_{0} C(X, E)$ can, at this point, be readily characterized as the relative weighted topology induced by $C \bar{V}_{0}(X, E)$.

1.3. THEOREM. (a) If $X$ is a locally compact (Hausdorff) space, $E$ is a normed space, and $\mathfrak{V}=\left\{V_{n}\right\}_{n \in \mathrm{N}}$ is a sequence of systems of weights on $X$ with $V_{n+1} \leqslant V_{n}$ for each $n \in \mathbf{N}$, then the canonical injection from $\mathfrak{V}_{0} C(X, E)$ into $C \bar{V}_{0}(X, E)$ is a topological isomorphism.

(b) Assume, moreover, that $E$ is a Banach space and $\mathscr{K} \leqslant V_{n}, n=1,2, \ldots$ Then $C \bar{V}_{0}(X, E)$ is the completion of $\mathcal{V}_{0} C(X, E)$.

(c) Under the conditions of (b), if, additionally, $\mathcal{V}=\left\{V_{n}\right\}_{n}$ satisfies condition (V) (of $0.4)$ and $C V_{n}(X, E)$ is a (DF)-space for each $n \in \mathbf{N}$, then

$$
\mathcal{V} C(X, E)=\mathcal{V}_{0} C(X, E)=C \bar{V}_{0}(X, E)
$$

as topological vector spaces.

(d) Let $X$ be locally compact and $E$ be a Banach space. If $V_{n}=\left\{\lambda v_{n} ; \lambda>0\right\}$ for $a$ decreasing sequence of weights $v_{n}$ on $X$ such that $\inf \left\{v_{n}(x) ; x \in K\right\}>0$ for each compact subset $K$ of $X, n=1,2, \ldots$, and

(V) for every $n \in \mathbf{N}$ there exists $m>n$ such that $v_{m} / v_{n}$ vanishes at infinity, then the algebraic and topological identity $\mathcal{V}_{0} C(X, E)=C \bar{V}_{0}(X, E)$ is valid, and hence $C \bar{V}_{0}(X, E)$ is an ultrabornological $(D F)$-space.

Proof. (a) In view of Lemma 1.1, it suffices to apply Lemma 1.2 with $\left(E_{1}, \tau_{1}\right)=$ $C \bar{V}_{0}(X, E),\left(E_{2}, \tau_{2}\right)=\mathcal{V}_{0} C(X, E)$, and $D=C_{c}(X, E)$.

(b) In this case, $\mathscr{K} \subset \bar{V}$, whereby $C \bar{V}_{0}(X, E)$ is complete for any complete 1.c. space $E$. Since $\Upsilon_{0} C(X, E) \supset C_{c}(X, E)$ and thereby is dense in $C \bar{V}_{0}(X, E)$, it remains to apply (a).

(c) and (d) Using 0.4.(c) (only for locally compact spaces $X$ which are necessarily countable at infinity here), we can conclude (c) from (b). The assertion (d) is nothing but a reformulation of (c) under slightly stronger hypotheses, but it is in this form that $1.3(\mathrm{c})$ is usually applied in concrete examples. 
1.4. Corollary. (a) Under the conditions of 1.3(a), $\mathfrak{V}_{0} C(X, E)$ is a topological linear subspace of $\mathfrak{V} C(X, E)$ which has the approximation property (a.p.) whenever the normed space $E$ does.

(b) In case $E=\mathbf{C}$, if l denotes a continuous linear functional on $\mathcal{V}_{0} C(X)$, then there exist a bounded Radon measure $\mu$ on $X$ and a weight $v \in \bar{V}$ such that

$$
l(f)=\int_{X} f v d \mu, \quad f \in \mathcal{V}_{0} C(X)
$$

conversely, for any bounded Radon measure $\mu$ on $X$ and any $v \in \bar{V}$, (*) defines a continuous linear functional l on $\mathfrak{V}_{0} C(X)$.

(c) Under the conditions of 1.3(b), suppose that $\mathfrak{V}=\left\{V_{n}\right\}_{n}$ with $V_{n}=\left\{\lambda v_{n} ; \lambda>0\right\}$ for a decreasing sequence of weights $v_{n}$ (such that $\inf \left\{v_{n}(x) ; x \in K\right\}>0$ for each compact subset $K$ of $X$ and $n=1,2, \ldots)$. Then $C \bar{V}_{0}(X, E)$ is a barrelled $(D F)$-space.

Proof. (a) Since the canonical injections $\mathscr{V}_{0} C(X, E) \rightarrow \mathcal{V} C(X, E) \rightarrow C \bar{V}(X, E)$ are continuous and $C \bar{V}(X, E)$ induces the weighted topology on $C \bar{V}_{0}(X, E)$, the first assertion follows immediately from 1.3(a). Since $C_{c}(X, E) \subset \mathcal{V}_{0} C(X, E), 1.3$ (a) implies that $\mathfrak{V}_{0} C(X, E)$ is a dense topological linear subspace of $C \bar{V}_{0}(X, E)$. From Corollary 2 in [27] (also see [5.I.]), $C \bar{V}_{0}(X, E)$ has the a.p. whenever $E$ does, and this leads to the desired conclusion because the a.p. is inherited by dense subspaces.

(b) $\mathcal{V}_{0} C(X)$ is a dense topological linear subspace of $C \bar{V}_{0}(X)$, and hence $\mathcal{V}_{0} C(X)^{\prime}$ $=C \bar{V}_{0}(X)^{\prime}$. The result now follows as a consequence of known representation theorems for the elements of $C \bar{V}_{0}(X)^{\prime}$; cf. [14] (also see [35] or [22]).

(In the same way, of course, similar characterizations of continuous linear functionals on $C \bar{V}_{0}(X, E)$ yield analogous representation theorems for $\mathcal{V}_{0} C(X, E)^{\prime}$, where $E$ is an arbitrary normed space.)

(c) This follows from $1.3(\mathrm{~b})$ because $\mathfrak{V}_{0} C(X, E)$ is here an ultrabornological $(D F)$-space, and the completion of a barrelled $(D F)$-space inherits these properties.

1.5. Remark. In [7, 2.3], a modification of an idea due to G. Köthe (in the context of sequence spaces) led to the following example. For the open unit disk $X$ of $\mathbf{C}$, we take $\mathfrak{V}=\left\{v_{n}\right\}_{n}$, where the continuous weights $v_{n}$ on $X$ are defined by

$$
v_{n}(r, \varphi)=\left\{\begin{array}{llll}
1, & r=0 & \text { or } & 0<r<1 \text { and } \varphi \in\left[-\frac{1}{n}, \frac{1}{n}\right] \\
1-r, & 0<r<1 & \text { and } & \varphi \notin\left(-\frac{2}{n}, \frac{2}{n}\right) \\
1+r n\left(\frac{1}{n}+\varphi\right), & 0<r<1 & \text { and } \quad \varphi \in\left(-\frac{2}{n},-\frac{1}{n}\right) \\
1-r n\left(\varphi-\frac{1}{n}\right), & 0<r<1 & \text { and } \quad \varphi \in\left(\frac{1}{n}, \frac{2}{n}\right) & n=1,2, \ldots
\end{array}\right\},
$$

Then $\mathfrak{V}_{0} C(X)=$ ind $_{n \rightarrow} C\left(v_{n}\right)_{0}(X)$ is not a regular inductive limit and hence not even sequentially complete. In view of $1.3(\mathrm{~b}), \mathfrak{V}_{0} C(X)$ must be a proper (dense 
topological) subspace of its associated space $C \bar{V}_{0}(X)$. This example then demonstrates that the assumptions of $1.4(\mathrm{c})$ are not sufficient-not even in the scalar case $E=\mathbf{C}$ and not even with continuous weights $v_{n}, n=1,2, \ldots$-for the identity $\mathfrak{V}_{0} C(X, E)=C \bar{V}_{0}(X, E)$.

On the other hand, as we have shown in 1.3(d), it does suffice to add condition (V) for the case of Banach spaces $E$. A more detailed investigation of conditions for completeness of $\mathfrak{T}_{0} C(X, E)$ (and hence for the identity $\mathfrak{V}_{0} C(X, E)=C \bar{V}_{0}(X, E)$ ) without $(\mathrm{V})$, but in the restricted setting that $E$ is a Banach space and $\mathfrak{V}=\left\{v_{n}\right\}_{n}$, is undertaken in the next section; Theorem 2.3 will improve upon Theorem 1.3(d) considerably.

1.6. THEOREM. Let $X$ be a locally compact space and take $\mathfrak{V}=\left\{v_{n}\right\}_{n}$ to be a decreasing sequence of weights $v_{n}$ on $X$ such that $\inf \left\{v_{n}(x) ; x \in K\right\}>0$ for each compact subset $K$ of $X, n=1,2, \ldots$ Further, we suppose that condition $(\mathrm{V})$ holds and that $(A(X), c 0)$ is a semi-Montel space. Then, as topological vector spaces,

$$
\checkmark A(X)=\Upsilon_{0} A(X)=A \bar{V}_{0}(X)
$$

Hence $A \bar{V}_{0}(X)$ is a Silva space, and here Corollary $1.4(\mathrm{~b})$ applies as well to $\widetilde{V}_{0} A(X)$. In particular, this holds when $X$ is an arbitrary open subset of $\mathbf{C}^{N}, N \geqslant 1$, and $A(X)=H(X)$ is the space of all holomorphic functions on $X$.

Proof. 0.4(d) implies that the Silva space $\checkmark A(X)=\mho_{0} A(X)$ is a topological linear subspace of $\widetilde{ } C(X)=\Upsilon_{0} C(X)$ under our assumptions, and since $C \bar{V}_{0}(X)$ induces the weighted topology on $A \bar{V}_{0}(X)$, we obtain the desired conclusion from 1.3(d) by taking intersections with the space $A(X)$. For the remark on Corollary 1.4(b), it suffices to apply the Hahn-Banach theorem.

Theorem 1.6 gives a new, purely functional analytic and rather easy proof of a theorem due to B. A. Taylor [37] on "complex representations" for spaces of entire functions and its extension to arbitrary open subsets $X$ of $\mathbf{C}^{N}$. For a more detailed discussion of Taylor's theorem, a comparison of the methods, and applications (above all, in relation to the theory of analytically uniform spaces due to $\mathrm{L}$. Ehrenpreis [11]), we refer to $\$ 4$.

Clearly, in the general situation of 1.3(a), if $A(X, E)$ is a subspace of $C(X, E)$; the canonical injection of $\Upsilon_{0} A(X, E)$ into $A \bar{V}_{0}(X, E)$ is a topological isomorphism whenever $\mathfrak{V}_{0} A(X, E)$ is a topological linear subspace of $\mathfrak{V}_{0} C(X, E)$. Since we would then have $\mho_{0} A(X, E)=A \bar{V}_{0}(X, E)$ algebraically and topologically if $\mho_{0} C(X, E)=$ $C \bar{V}_{0}(X, E)$ as vector spaces, any positive solution of the Problem in $\S 0.4$ will provide information about the topologies of $\mho_{0} A(X, E)$ and $A \bar{V}_{0}(X, E)$.

Having derived our general results, we now proceed to show that, in the setting of Theorem 1.3(d) and Theorem 1.6, respectively, we may restrict our attention from the maximal system $\bar{V}$ to a subsystem $\hat{V}$ of weights of a rather special form. Again, we will utilize continuous partitions of unity, but this time countable ones on the whole space $X$.

1.7. Definition. Let $X$ denote a locally compact and $\sigma$-compact space and let $\mathfrak{V}=\left\{v_{n}\right\}_{n \in \mathbf{N}}$ be a decreasing sequence of weights on $X$ such that $\inf \left\{v_{n}(x)\right.$; $x \in K\}>0$ for each compact subset $K$ of $X$ and $n=1,2, \ldots$; we fix a sequence 
$\left(K_{n}\right)_{n}$ of compact subsets of $X$ such that $K_{n} \subset \stackrel{\circ}{K}_{n+1}, n=1,2, \ldots$, and $X=$ $\cup_{n \in \mathrm{N}} K_{n}$.

Let $a=(a(n))_{n \in \mathrm{N}}$ denote an arbitrary strictly increasing sequence of natural numbers. Since $X$ is paracompact, we may now choose a continuous partition of unity $\left(\varphi_{n}^{(a)}\right)_{n}$ which is subordinate to the covering $\left(\dot{K}_{a(n+1)} \backslash K_{a(n-1)}\right)_{n}$ of $X$, where $a(0)=0$ and $K_{0}=\varnothing$, and for which $\varphi_{1}^{(a)}$ is strictly positive on $K_{a(1)}$. For a constant $c>0$, we set $v_{0} \equiv c$ on $X$ and define

$$
\bar{v}_{a, c}(x)=\sum_{k=1}^{\infty} v_{k-1}(x) \varphi_{k}^{(a)}(x), \quad x \in X .
$$

1.8. LEMMA. Every $\bar{v}_{a, c}$ is a weight in $\bar{V}$ (which is strictly positive on $X$ ).

Proof. $\bar{v}_{a, c}$ is clearly well-defined. Since at most three terms in the sum on the right-hand side of its definition do not vanish on $\stackrel{\circ}{K}_{a(n+1)} \backslash K_{a(n-1)}, n=1,2, \ldots, \bar{v}_{a, c}$ is a weight on $X$ which is continuous whenever all $v_{n}, n=1,2, \ldots$, are continuous.

To show $\bar{v}_{a, c} \in \bar{V}$, fix $n \in \mathbf{N}$. For each $x \notin K_{a(n+1)}, \varphi_{k}^{(a)}(x)=0$ for all $k \leqslant n$ and hence

$$
\frac{\bar{v}_{a, c}(x)}{v_{n}(x)}=\sum_{k=n+1}^{\infty} \varphi_{k}^{(a)}(x) \frac{v_{k-1}(x)}{v_{n}(x)} \leqslant \sum_{k=n+1}^{\infty} \varphi_{k}^{(a)}(x) \leqslant 1
$$

because $\left\{v_{n}\right\}_{n}$ is decreasing. But on $K_{a(n+1)}, \varphi_{k}^{(a)}$ vanishes for each $k \geqslant n+2$ and the u.s.c. functions $v_{0}, v_{1}, \ldots, v_{n}$ are bounded by some constant $M>0$. Hence, for all $x \in K_{a(n+1)}$,

$$
\frac{\bar{v}_{a, c}(x)}{v_{n}(x)}=\sum_{k=1}^{n+1} \varphi_{k}^{(a)}(x) \frac{v_{k-1}(x)}{v_{n}(x)} \leqslant \frac{M}{\inf \left\{v_{n}(x) ; x \in K_{a(n+1)}\right\}} ;
$$

i.e., $\bar{v}_{a, c} / v_{n}$ is bounded on $X$. Since $n \in \mathbf{N}$ was arbitrary, we have established our claim.

We will now let $\hat{V}=\hat{V}_{\mho}$ denote the subset of $\bar{V}=\bar{V}_{\checkmark}$ which consists of all weights of the form $\bar{v}_{a, c}$ where $a=(a(n))_{n}$ runs through all strictly increasing sequences of positive integers and $c$ is an arbitrary positive constant.

Supposing that $X$ is locally compact and the sequence $\mathcal{V}$ satisfies $(\mathrm{V})$, in which case $X$ is necessarily countable at infinity (see 0.4 ), we would prove $\bar{V} \leqslant \hat{V}$.

To this end, fix $v \in \bar{V}$. Given $n \in \mathbf{N}$, if $m>n$ is chosen as in condition (V), $v_{m} / v_{n}$ vanishes at infinity, and since $v / v_{m}$ is bounded, $v / v_{n}$ also vanishes at infinity. By induction, we may therefore choose a strictly increasing sequence $a=(a(n))_{n}$ of natural numbers such that

$$
\frac{v(x)}{v_{n}(x)} \leqslant 1 \quad \text { for all } x \notin K_{a(n-1)}, \quad n=2,3, \ldots
$$

Taking a corresponding continuous partition of unity $\left(\varphi_{k}^{(a)}\right)_{k}$ and a constant

$$
c \geqslant \max \left(\sup \left\{v_{2}(y) ; y \in K_{a(2)}\right\}, \frac{\sup \left\{v(y) ; y \in K_{a(1)}\right\}}{\inf \left\{\varphi_{1}^{(a)}(y) ; y \in K_{a(1)}\right\}}\right),
$$

we claim that $v \leqslant \bar{v}_{a, c}$. 
In fact, if $x \in K_{a(1)}$,

$$
v(x) \leqslant c \inf \left\{\varphi_{1}^{(a)}(y) ; y \in K_{a(1)}\right\} \leqslant v_{0}(x) \varphi_{1}^{(a)}(x) \leqslant \bar{v}_{a, c}(x),
$$

while $x \in K_{a(2)} \backslash K_{a(1)}$ implies $\varphi_{k}^{(a)}(x)=0$ for all $k \geqslant 3$, and hence by our choice of $c$,

$$
\begin{aligned}
v(x) & \leqslant v_{2}(x)=\sum_{k=1}^{2} \varphi_{k}^{(a)}(x) v_{2}(x) \leqslant \varphi_{1}^{(a)}(x) c+\varphi_{2}^{(a)}(x) v_{1}(x) \\
& =\sum_{k=1}^{2} v_{k-1}(x) \varphi_{k}^{(a)}(x)=\bar{v}_{a, c}(x) .
\end{aligned}
$$

Otherwise, there exists $n \geqslant 3$ such that $x \in \stackrel{\circ}{K}_{a(n+1)} \backslash K_{a(n-1)}$. Since then $\varphi_{k}^{(a)}(x)=0$ for all $k \notin\{n-1, n, n+1\}$, we have

$$
v(x) \leqslant v_{n}(x)=\sum_{k=n-1}^{n+1} \varphi_{k}^{(a)}(x) v_{n}(x) \leqslant \sum_{k=n-1}^{n+1} \varphi_{k}^{(a)}(x) v_{k-1}(x)=\bar{v}_{a, c}(x) .
$$

But $\bar{V} \leqslant \hat{V} \subset \bar{V}$ obviously implies that $\hat{V}$ is a system of weights on $X$ and that $A \bar{V}_{0}(X, E)=A \hat{V}_{0}(X, E)$ holds for an arbitrary 1.c. space $E$ and an arbitrary subspace $A(X, E) \subset C(X, E)$. Thus, we have proved the following result.

1.9. Proposition. In Theorem 1.3(d) and Theorem 1.6, the maximal system $\bar{V}=\bar{V}_{\mathscr{v}}$ associated with $\mathcal{V}$ can be replaced by

$$
\hat{V}=\left\{\bar{v}_{a, c} ; a=(a(n))_{n} \text { strictly increasing sequence of positive integers, } c>0\right\} \text {, }
$$

where $\bar{v}_{a, c}$ is defined as in 1.7 .

Consequently, under the conditions of 1.3(d) and 1.6, respectively, the weights $v$ which occur in the integral representation theorem 1.4(b) for $\mathfrak{T}_{0} C(X)^{\prime}$ and $\mathfrak{V}_{0} A(X)^{\prime}$, respectively, may all be taken from $\hat{V}$ instead of $\bar{V}$. If all the weights $v_{n}, n=1,2, \ldots$, are continuous, $\hat{V} \subset \tilde{V}$. Hence $\bar{V}$ may be replaced by $\tilde{V}$ in Theorems $1.3(\mathrm{~d})$ and 1.6, and in Corollary 1.4(b) under the conditions of 1.3(d), whenever the original system $\checkmark$ consists of continuous weights $v_{n}, n=1,2, \ldots$ (This last consequence, however, follows more directly from the proposition in 0.2 .)

Of course, our construction also allows for other reductions; we will here note a particularly obvious one.

1.10. REMARK. Let $X$ denote an open subset of $\mathbf{C}^{N}\left(\cong \mathbf{R}^{2 N}\right), N \geqslant 1$, and suppose that all $v_{n}, n=1,2, \ldots$, are $C^{\infty}$-functions. Then we may take $C^{\infty}$-partitions of unity $\left\{\varphi_{k}^{(a)}\right\}_{k \in \mathbf{N}}$ in the definition of $\bar{v}_{a, c}$, and so it turns out that it suffices to consider only infinitely differentiable weights (of the form $\bar{v}_{a, c}$ ). As a consequence, for the three results which we mentioned at the end of 1.9 , in this case $\bar{V}$ may also be replaced by a subsystem of $C^{\infty}$-functions.

At this point, let us also add that, for open subsets of $\mathbf{C}^{N}$, more precise results and certain additional properties of the weights in $\hat{V}$ may be obtained if locally finite open coverings of $X$ and subordinate partitions of unity are constructed using the method of Whitney (e.g., see Pedersen [26, p. 399]) instead of the general approach of Definition 1.7. 
Proposition 1.9, well understood, clearly opens the possibility of proving Theorem 1.3(d) using only weights in $\hat{V}$. We note such a proof below; it is direct, quite different from our first, demonstrates another point of view, and also shows immediately that it is not necessary to assume completeness of the normed space $E$ in $1.3(\mathrm{~d})$.

Direct proof of Theorem 1.3(d) for an arbitrary normed space $E$.

(i) Firstly, we claim that $\mathcal{V} C(X, E)=C \bar{V}(X, E)$ algebraically. To prove this, we will assume only that $X$ is locally compact and $\sigma$-compact; condition (V) is not needed here. (Also see [7, 2.8]; more general, and more precise, results of this type will be given in $\$ 5$ below.)

Fix $f \in C \bar{V}(X, E)$; we would show that $q_{v_{n}}(f)=\sup _{x \in X} v_{n}(x)\|f(x)\|<\infty$ for some $n \in \mathbf{N}$. If this is not the case, then, given $n \in \mathbf{N}$ and $C_{n}>0$, there exists $y=y\left(n, C_{n}\right)$ such that $v_{n}(y)\|f(y)\|>C_{n}$. Now, $X=\cup_{n \in \mathbf{N}} K_{n}$ where $K_{n}$ is compact and $K_{n} \subset K_{n+1}, n=1,2, \ldots$. Therefore, since $v_{n} f$ is bounded on compact subsets of $X$, a simple induction argument yields a strictly increasing sequence $a=(a(n))_{n}$ of positive integers so that, for some point $x_{n} \in \stackrel{\circ}{K}_{a(n+1)} \backslash K_{a(n-1)}, v_{n}\left(x_{n}\right)\left\|f\left(x_{n}\right)\right\|>n$, $n=1,2, \ldots\left(a(0)=0, K_{0}=\varnothing\right)$.

Construct the weight $\bar{v}_{a, c}=\sum_{k=1}^{\infty} v_{k-1} \varphi_{k}^{(a)} \in \hat{V} \subset \bar{V}$ as described in 1.7, where $c \geqslant \max \left\{v_{1}\left(x_{1}\right), v_{2}\left(x_{2}\right)\right\}$. Then,

$$
\begin{aligned}
\bar{v}_{a, c}\left(x_{1}\right) & =\sum_{k=1}^{\infty} v_{k-1}\left(x_{1}\right) \varphi_{k}^{(a)}\left(x_{1}\right)=v_{0}\left(x_{1}\right) \varphi_{1}^{(a)}\left(x_{1}\right)+v_{1}\left(x_{1}\right) \varphi_{2}^{(a)}\left(x_{1}\right) \\
& \geqslant v_{1}\left(x_{1}\right) \sum_{k=1}^{2} \varphi_{k}^{(a)}\left(x_{1}\right)=v_{1}\left(x_{1}\right), \\
\bar{v}_{a, c}\left(x_{n}\right) & =\sum_{k=1}^{\infty} v_{k-1}\left(x_{n}\right) \varphi_{n}^{(a)}\left(x_{n}\right)=\sum_{k=n-1}^{n+1} v_{k-1}\left(x_{n}\right) \varphi_{k}^{(a)}\left(x_{n}\right) \\
& \geqslant \sum_{k=n-1}^{n+1} v_{n}\left(x_{n}\right) \varphi_{k}^{(a)}\left(x_{n}\right)=v_{n}\left(x_{n}\right), \quad n=2,3, \ldots
\end{aligned}
$$

Hence

$$
q_{\bar{v}_{a, c}}(f) \geqslant \bar{v}_{a, c}\left(x_{n}\right)\left\|f\left(x_{n}\right)\right\| \geqslant v_{n}\left(x_{n}\right)\left\|f\left(x_{n}\right)\right\|>n
$$

for each $n \in \mathbf{N}$, which obviously contradicts $f \in C \bar{V}(X, E)$.

(ii) The inductive limit topology of $\mathcal{V} C(X, E)$ is finer than the weighted topology of $C \bar{V}(X, E)$, and, subject to condition (V), we want to show that the converse holds as well. In view of condition (V) (and reducing the sequence $\left\{v_{n}\right\}_{n \in N}$ if necessary), we may assume without loss of generality that $v_{n+1} / v_{n}$ vanishes at infinity for $n=1,2, \ldots$ We now observe that each neighborhood of 0 in $\mathcal{T} C(X, E)$ contains a set

$$
U=\overline{\Gamma\left(\bigcup_{n=1}^{\infty} U_{n}\right)}
$$


with

$$
U_{n}=\left\{f \in C v_{n}(X, E) ; q_{v_{n}}(f)=\sup _{x \in X} v_{n}(x)\|f(x)\|<\rho_{n}\right\}, \quad n=1,2, \ldots,
$$

where $\left(\rho_{n}\right)_{n}$ is a decreasing sequence of positive numbers and the closure is taken in $\mathfrak{V} C(X, E)$.

Fix a sequence $\left(K_{n}\right)_{n \in \mathrm{N}}$ of compact subsets of $X$ with $X=\cup_{n \in \mathrm{N}} K_{n}$ and $K_{n} \subset \stackrel{\circ}{K}_{n+1}, n=1,2, \ldots$. Inductively, we can find a strictly increasing sequence $a=(a(n))_{n \in \mathrm{N}}$ of positive integers such that

$$
\frac{v_{n+1}(x)}{v_{n}(x)}<\frac{\rho_{n+1}}{2^{n+1}} \quad \text { for all } x \notin K_{a(n)},
$$

$n=1,2, \ldots$ Now, put $a(0)=0, K_{0}=\varnothing, v_{0} \equiv c=1$, and take a continuous partition of unity $\left(\varphi_{n}^{(a)}\right)_{n}$ subordinate to the covering $\left(\stackrel{\circ}{K}_{a(n+1)} \backslash K_{a(n-1)}\right)_{n \in \mathbf{N}}$. We define $\bar{v}=\bar{v}_{a, c}=\sum_{k=1}^{\infty} v_{k-1} \varphi_{k}^{(a)} \in \hat{V} \subset \bar{V}$ (cf. 1.7, 1.8). At this point, it suffices to show that, for

$$
\bar{U}=\left\{f \in C \bar{V}(X, E) ; q_{\bar{v}}(f)=\sup _{x \in X} \bar{v}(x)\|f(x)\|<\min \left(1, \frac{\rho_{1}}{2 \sup _{x \in K_{a(2)}} v_{1}(x)}\right)\right\},
$$

$\bar{U} \subset U$.

To do so, let us first note that any $f \in C(X, E)$ can be represented in the following way:

$$
f=\sum_{n=1}^{\infty} \varphi_{n}^{(a)} f=\sum_{n=1}^{\infty} \frac{1}{2^{n}} f_{n}, \quad f_{n}=2^{n} \varphi_{n}^{(a)} f
$$

where the series converges uniformly on compact subsets of $X$. Moreover, if $f$ belongs to $C \bar{V}(X, E)$, then the first part of the present proof shows that there exists $n_{0} \in \mathbf{N}$ so that $f \in C v_{n_{0}}(X, E)$. But, in this case, with respect to the norm $q_{v_{n_{0}}}$, the partial sums of the series $\Sigma_{n} f_{n} / 2^{n}$ are all bounded by $q_{v_{n_{0}}}(f)$, and so $0.4(\mathrm{a})$ implies that the series also converges with respect to the inductive limit topology of $\mathcal{V} C(X, E)$. Hence, the proof of our claim is reduced to showing that $f \in \bar{U}$ implies $f_{n} \in U_{n}, n=1,2, \ldots$; i.e.,

$$
q_{\bar{v}}(f)<\min \left(1, \frac{\rho_{1}}{2 \sup \left\{v_{1}(x) ; x \in K_{a(2)}\right\}}\right) \text { implies } q_{v_{n}}\left(f_{n}\right)<\rho_{n},
$$

which we verify by the following straightforward computation:

$$
\begin{aligned}
\sup _{x \in X} v_{1}(x)\left\|f_{1}(x)\right\| & =\sup _{x \in K_{a(2)}} v_{1}(x) 2 \varphi_{1}^{(a)}(x)\|f(x)\| \\
& \leqslant 2\left(\sup _{x \in K_{a(2)}} v_{1}(x)\right)\left(\sup _{x \in X} v_{0}(x) \varphi_{1}^{(a)}(x)\|f(x)\|\right) \\
& \leqslant 2\left(\sup _{x \in K_{a(2)}} v_{1}(x)\right)\left(\sup _{x \in X} \sum_{k=1}^{\infty} v_{k-1}(x) \varphi_{k}^{(a)}(x)\|f(x)\|\right) \\
& =2\left(\sup _{x \in K_{a(2)}} v_{1}(x)\right) q_{\bar{v}}(f)<\rho_{1},
\end{aligned}
$$


while, for $n=1,2, \ldots$,

$$
\begin{aligned}
\sup _{x \in X} v_{n+1}(x)\left\|f_{n+1}(x)\right\| & =\sup _{x \in X \backslash K_{a(n)}} \frac{v_{n+1}(x)}{v_{n}(x)} v_{n}(x) 2^{n+1} \varphi_{n+1}^{(a)}(x)\|f(x)\| \\
& \leqslant \frac{\rho_{n+1}}{2^{n+1}} 2^{n+1} \sup _{x \in X} v_{n}(x) \varphi_{n+1}^{(a)}(x)\|f(x)\| \\
& \leqslant \rho_{n+1} \sup _{x \in X} \sum_{k=1}^{\infty} v_{k-1}(x) \varphi_{k}^{(a)}(x)\|f(x)\|=\rho_{n+1} q_{\bar{v}}(f)<\rho_{n+1} .
\end{aligned}
$$

Finally, recall that condition (V) implies $\mathscr{V} C(X, E)=\mathcal{V}_{0} C(X, E)$, while the identity $C \bar{V}(X, E)=C \bar{V}_{0}(X, E)$ can be deduced as follows: Fix $f \in C \bar{V}(X, E)$ and $v \in \bar{V}$; by part (i) of the proof, there exists $n \in \mathbf{N}$ so that $f \in C v_{n}(X, E)$. Now, since $v_{n+1} / v_{n}$ vanishes at infinity and $v / v_{n+1}$ is bounded, $v f=\left(v / v_{n+1}\right)\left(v_{n+1} / v_{n}\right) v_{n} f$ vanishes at infinity; i.e., $f \in C \bar{V}_{0}(X, E)$.

Let us note two refinements which follow from the preceding proof.

1.11. REMARK. (a) Theorem 1.3(d) holds for an arbitrary normed space $E$.

(b) In 1.3(d) and 1.6, we also have $C \bar{V}_{0}(X, E)=C \bar{V}(X, E)$ and $A \bar{V}_{0}(X)=A \bar{V}(X)$, respectively.

Furthermore, let us assume that $X$ is locally compact and $\sigma$-compact, that $E$ is a normed space, and that $\mathcal{V}=\left\{v_{n}\right\}_{n \in \mathbf{N}}$ is a decreasing sequence of weights $v_{n}$ on $X$ such that $\inf \left\{v_{n}(x) ; x \in K\right\}>0$ for each compact subset $K$ of $X, n=1,2, \ldots$ Then a slight refinement of part (i) in the direct proof of Theorem 1.3(d) (see again [7, 2.8]) not only shows that $\mathcal{V} C(X, E)=C \bar{V}(X, E)$ algebraically, but also that the two spaces $\mathfrak{V} C(X, E)$ and $C \bar{V}(X, E)$ have the same bounded sets.

To verify this, let $B$ be a bounded subset of $C \bar{V}(X, E)$ with its weighted topology and define $M(x)=\sup \{\|f(x)\| ; f \in B\}, x \in X$. Then, replacing $f \in C \bar{V}(X, E)$ by $M$ in (i) of the above proof ( $B$ is uniformly bounded on compact subsets of $X$ ), we see that there exists $n \in \mathbf{N}$ such that $\sup \left\{v_{n}(x) M(x) ; x \in X\right\}<\infty$ whence $B$ is a bounded subset of $C v_{n}(X, E)$ and hence bounded in $\mathscr{V} C(X, E)$. (By the way, this argument also shows that, in our case, $\mathscr{} C(X, E)$ is always a regular inductive limit.)

Thus, the situation can be described as follows: The two spaces $\mathscr{V} C(X, E)$ and $C \bar{V}(X, E)$ are equal algebraically, have the same bounded sets, and both induce the inductive limit topology on $\mathcal{V}_{0} C(X, E)$ (see 1.3(a) and 1.4(a)). It is then natural to ask whether the topologies of $\widetilde{ } C(X, E)$ and $C \bar{V}(X, E)$ are always identical as well. However, a negative answer to this question follows easily from a famous counterexample of Köthe and Grothendieck (see [23, I, §31, 7]).

1.12. Example. Let $X$ denote the discrete space $\mathbf{N} \times \mathbf{N}$, define $B=\left(b_{n}\right)_{n \in \mathbf{N}}$ and $\mathcal{V}=\left\{v_{n}\right\}_{n \in \mathbf{N}}$ by

$$
\begin{aligned}
& b_{n}(i, j)=\left\{\begin{array}{ll}
j, & 1 \leqslant i \leqslant n, \quad j=1,2, \ldots \\
1, & i>n, \quad j=1,2, \ldots
\end{array}\right\} \quad \text { and } \\
& v_{n}(i, j)=\frac{1}{b_{n}(i, j)}, \quad i, j=1,2, \ldots
\end{aligned}
$$


Then $\mathcal{} C(X)=C \bar{V}(X)$ algebraically (the two spaces have even the same bounded sets and induce the inductive limit topology on $\mathcal{V}_{0} C(X)$ ), but the inductive limit topology of $\mathscr{V} C(X)$ is strictly stronger than the weighted topology of $C \bar{V}(X)$.

Proof. Put

$\Lambda(B)=\left\{x=\left(x_{i j}\right)_{i, j \in \mathbf{N}} \in \mathbf{C}^{\mathbf{N} \times \mathbf{N}} ; p_{n}(x)=\sum_{i, j=1}^{\infty}\left|x_{i j}\right| b_{n}(i, j)<\infty\right.$ for each $\left.n \in \mathbf{N}\right\}$.

Under the natural ("normal") 1.c. topology given by the sequence $\left(p_{n}\right)_{n \in \mathbf{N}}$ of seminorms, $\Lambda(B)$ is clearly a ("perfect") Fréchet space ("gestufter Raum"), and its dual $\Lambda(B)^{\prime}$ ("Stufenraum") equals

$$
\bigcup_{n \in \mathbf{N}}\left\{y=\left(y_{i j}\right)_{i, j \in \mathbf{N}} \in \mathbf{C}^{\mathbf{N} \times \mathbf{N}}=C(X) ; \sup _{i, j \in \mathbf{N}}\left|y_{i j}\right| v_{n}(i, j)<\infty\right\}=\mathscr{V} C(X) .
$$

(See [23, I, §30, 8.(1)].) We claim that the strong topology $\beta\left(\Lambda(B)^{\prime}, \Lambda(B)\right)$ of $\Lambda(B)_{b}^{\prime}$ is stronger than the weighted topology of $C \bar{V}(X)(=\mathcal{V} C(X)$ algebraically).

An arbitrary neighborhood of 0 in $C \bar{V}(X)$ always contains a set

$$
\left\{y=\left(y_{i j}\right)_{i j} \in C \bar{V}(X) ; \sup _{i, j}\left|y_{i j}\right| \bar{v}(i, j) \leqslant 1\right\}
$$

for some $\bar{v} \in \bar{V}$, where we may assume by 1.8 that $\bar{v}$ is strictly positive on $X$. Since $\bar{v}$ belongs to $\bar{V}$, given $n \in \mathbf{N}$, there exists $\lambda_{n}>0$ such that $\bar{v} \leqslant \lambda_{n} v_{n}$. Putting

$$
A=\left\{x=\left(x_{i j}\right)_{i j} \in \mathbf{C}^{\mathbf{N} \times \mathbf{N}} ; \sum_{i, j=1}^{\infty} \frac{\left|x_{i, j}\right|}{\bar{v}(i, j)} \leqslant 1\right\} \text {, }
$$

we see that $A$ is bounded in $\Lambda(B)$ because for each $n \in \mathbf{N}$ and each $x \in A$

$$
p_{n}(x)=\sum_{i, j}\left|x_{i j}\right| b_{n}(i, j)=\sum_{i, j} \frac{\left|x_{i j}\right|}{v_{n}(i, j)} \leqslant \sum_{i, j} \frac{\left|x_{i j}\right|}{\bar{v}(i, j)} \lambda_{n} \leqslant \lambda_{n} .
$$

But an easy calculation shows that the polar $A^{0}$ of $A$ in $\Lambda(B)^{\prime}$ is nothing but

$$
\left\{y=\left(y_{i j}\right)_{i j} \in \Lambda(B)^{\prime} ; \sup _{i, j}\left|y_{i j}\right| \bar{v}(i, j) \leqslant 1\right\},
$$

which suffices to prove our claim.

Since the inductive limit topology of $\mathcal{T} C(X)$ is still stronger than $\beta\left(\Lambda(B)^{\prime}, \Lambda(B)\right)$, the identity mappings $\mathcal{V} C(X) \rightarrow \Lambda(B)_{b}^{\prime} \rightarrow C \bar{V}(X)$ are continuous. However, the space $\mathscr{V} C(X)$ is clearly bornological, while it is proved in [23, I, §31, 7] that $\Lambda(B)_{b}^{\prime}$ is not bornological. Thus the fact that $\mathscr{T} C(X)$ and $C \bar{V}(X)$ have the same bounded sets together with a well-known result from the theory of bornological spaces (e.g., see $[23, \mathrm{I}, \S 28,2])$ imply that $C \bar{V}(X)$ must also fail to be bornological wherefore the weighted topology is strictly weaker than the inductive limit topology.

This counterexample, when compared with the positive result 1.3(a) above, provides a degree of justification for restricting our attention to the case of $\mathcal{V}_{0} C(X, E)$ and $C \bar{V}_{0}(X, E)$ (instead of $\mathscr{V} C(X, E)$ and $\left.C \bar{V}(X, E)\right)$ in the present section. 
Example 1.12 is also quite interesting from another point of view. In particular, the Fréchet space $\Lambda(B)$ is not quasi-normable, while its strong dual $\Lambda(B)_{b}^{\prime}$ is complete, neither separable nor infrabarreled, contains nonmetrizable bounded subsets, and does not satisfy the strict Mackey convergence condition (cf. Grothendieck [15]). Moreover, the inductive limit topology of $\checkmark C(X)$ is that of ind $_{n \rightarrow} \Lambda(B)_{U_{n}^{0}}^{\prime}$, where

$$
U_{n}=\left\{x=\left(x_{i j}\right)_{i j} \in \mathbf{C}^{\mathbf{N} \times \mathbf{N}} ; p_{n}(x)=\sum_{i, j=1}^{\infty}\left|x_{i j}\right| b_{n}(i, j) \leqslant 1\right\}, \quad n=1,2, \ldots ;
$$

it is thus the bornological topology associated with $\beta\left(\Lambda(B)^{\prime}, \Lambda(B)\right)$, and hence we can conclude from $\left[23, \mathrm{I}, \S 29,4\right.$.(2)] that the topology of $\mathcal{} \mathcal{V} C(X)$ is $\beta\left(\Lambda(B)^{\prime}, \Lambda(B)^{\prime \prime}\right)$ wherefore $\mathscr{T} C(X)$ is complete.

In the case of sequence spaces, taking $X=\mathbf{N}$ and $\mathcal{V}=\left\{v_{n}\right\}_{n}$ to be a decreasing sequence of strictly positive weights on $\mathbf{N}, \mathcal{T} C(X)$ is always a co-echelon space ("Stufenraum") in the terminology of $[23, I, \S 30,8]$, being the dual of the ("perfect" Fréchet) echelon space ("gestufter Raum") $\Lambda(B)$ (under its "normal" topology), where $B=\left(b_{n}\right)_{n \in \mathrm{N}}$ and $b_{n}=1 / v_{n}, n=1,2, \ldots$ It is again easy to see that the identity mappings

$$
\mathcal{V} C(X)=\operatorname{ind}_{n \rightarrow} \Lambda(B)_{U_{n}^{0}}^{\prime} \rightarrow \Lambda(B)_{b}^{\prime} \rightarrow C \bar{V}(X)
$$

are continuous, where

$$
U_{n}=\left\{x=\left(x_{j}\right)_{j \in \mathbf{N}} ; \sum_{j=1}^{\infty}\left|x_{j}\right| b_{n}(j) \leqslant 1\right\}, \quad n=1,2, \ldots
$$

Further, exactly as above, $\mathcal{T} C(X)$ is the bornological space associated with $\Lambda(B)_{b}^{\prime}$, hence has the topology $\beta\left(\Lambda(B)^{\prime}, \Lambda(B)^{\prime \prime}\right)$, and therefore is complete.

As we shall next demonstrate, it is actually possible to establish completeness of the weighted spaces $\mathcal{T} A(X)$ under rather general conditions which include the above mentioned case for sequence spaces. Also, as the proof below demonstrates, the associated weighted space $A \bar{V}(X)$, even though it may fail to equal $\mathfrak{V} A(X)$ topologically, can still be put to good use in the topological theory of weighted inductive limits.

1.13. TheOREM. Let $X$ denote a locally compact space, and take $\mathfrak{V}=\left\{v_{n}\right\}_{n \in \mathrm{N}}$ to be a decreasing sequence of weights $v_{n}$ on $X$ with $\inf \left\{v_{n}(x) ; x \in K\right\}>0$ for each compact subset $K$ of $X, n=1,2, \ldots$. If the linear subspace $A(X)$ is closed in $(C(X)$, co) and if $(A(X), \mathrm{co})$ is a semi-Montel space, then $\mathfrak{T} A(X)$ is the barrelled space associated with $A \bar{V}(X)$ and hence is complete.

Proof. (For the facts about the associated bornological, ultrabornological and barrelled spaces which we need here, see the monograph by Schmets [29].)

From our remarks preceding Example 1.12, it follows that the two spaces $\mathscr{T} A(X)$ and $A \bar{V}(X)$ are equal algebraically and have the same bounded subsets whence, clearly, $\mathfrak{} A(X)$ is the bornological space associated with $A \bar{V}(X)$. (The additional hypothesis requiring $X$ to be $\sigma$-compact was inserted after Remark 1.11, but we will 
show in Proposition 5.8(a) below that this hypothesis is not necessary; cf. Corollary 5.12(a) as well.) Moreover, since each $A v_{n}(X)$ is a Banach space under our hypotheses, $\mathcal{V} A(X)$ is also the ultrabornological space associated with $A \bar{V}(X)$, and hence, letting $E_{t}$ denote the barrelled space associated with a l.c. space $E$, we have the continuous identity mappings

$$
\mathfrak{V} A(X) \rightarrow A \bar{V}(X)_{t} \rightarrow A \bar{V}(X) .
$$

At this point, we would apply a result due to Dineen [10, Proposition 3], taking

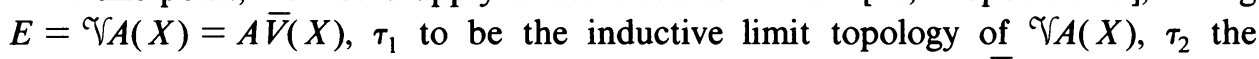
barrelled topology associated with the weighted topology of $A \bar{V}(X)$, and $\tau_{3}$ the compact-open topology co. Obviously, $\tau_{1} \geqslant \tau_{2} \geqslant \tau_{3},\left(E, \tau_{1}\right)$ is a bornological (DF)space, and $\left(E, \tau_{2}\right)$ is barrelled. The sequence $\left(B_{n}\right)_{n \in \mathbf{N}}$, where

$$
B_{n}=\left\{f \in A v_{n}(X) ; \sup _{x \in X} v_{n}(x)|f(x)| \leqslant n\right\}, \quad n=1,2, \ldots,
$$

forms a basis for the bounded sets in $\mathcal{V} A(X)$ because ind ${ }_{n \rightarrow} A v_{n}(X)$ is regular with ind $_{n \rightarrow} C v_{n}(X)$. But, since these sets are closed and bounded in the semi-Montel space $\left(A(X)\right.$, co), it follows that $B_{n}$ is closed in $\mathscr{V} A(X)$ and $\tau_{3}$-compact, $n=1,2, \ldots$ Now, applying [10, Proposition 3], we can conclude, as desired, that $\tau_{1}=\tau_{2}$. Since the barrelled space $E_{t}$ associated with a complete l.c. space $E$ is complete, we thus deduce the completeness of $\mathfrak{V} A(X)$ from the fact that $A \bar{V}(X)$ is complete.

Let us remark that we do not know whether $\mathcal{V} C(X)$ is always complete when $X$ is a locally compact Hausdorff space and $\mathcal{V}=\left\{v_{n}\right\}_{n \in \mathrm{N}}$ a decreasing sequence of weights $v_{n}$ on $X$ such that $\inf \left\{v_{n}(x) ; x \in K\right\}>0$ for each compact subset $K$ of $X$, $n=1,2, \ldots$. Clearly, in this situation, $\mathscr{T} C(X)$ is again the (ultra-) bornological space associated with the complete space $C \bar{V}(X)$, but we do not know whether the (weaker) associated barrelled topology (which yields a complete $(D F)$-space with the same bounded sets as $\mathscr{T}(X)$ ) will also coincide with the inductive limit topology. (Note that the sets

$$
B_{n}=\left\{f \in C v_{n}(X) ; \sup _{x \in X} v_{n}(x)|f(x)| \leqslant n\right\}, \quad n=1,2, \ldots,
$$

are closed and bounded, hence precompact, in $C(X)$ with respect to the topology of pointwise convergence on $X$.) Nonetheless, Theorem 1.13 does apply to the weighted inductive limit $\mathfrak{V} H(X)$ of holomorphic functions on an open subset $X$ of $\mathbf{C}^{N}$, as well as to spaces of type $\mathcal{V} C(X)$ when $X$ is discrete.

2. On $\mathcal{V}_{0} C(X, E)=C \bar{V}_{0}(X, E)$. Assuming that $X$ is a locally compact (Hausdorff) space, let $\mathscr{V}=\left\{v_{n}\right\}_{n \in \mathrm{N}}$ be a decreasing sequence of weights on $X$ such that $\inf \left\{v_{n}(x) ; x \in K\right\}>0$ for each compact subset $K$ of $X, n=1,2, \ldots$

2.1. Definition. We will say that $\mathfrak{V}$ is regularly decreasing if, given $n \in \mathbf{N}$, there exists $m \geqslant n$ so that, for every $\varepsilon>0$ and every $k \geqslant m$, it is possible to find $\delta(k, \varepsilon)>0$ with

$$
v_{k}(x) \geqslant \delta(k, \varepsilon) v_{n}(x) \text { whenever } v_{m}(x) \geqslant \varepsilon v_{n}(x) .
$$

In other words, $\mathcal{V}$ is regularly decreasing if, and only if, given $n \in \mathbf{N}$, there exists $m \geqslant n$ such that, on each subset of $X$ on which the quotient $v_{m} / v_{n}$ is bounded away from zero, also all quotients $v_{k} / v_{n}, k \geqslant m$, are bounded away from zero. 
2.2. Proposition. The sequence $\mathfrak{V}=\left\{v_{n}\right\}_{n \in \mathrm{N}}$ is regularly decreasing if, and only if, the following condition is satisfied:

$(\mathrm{wV})$ For every $n \in \mathbf{N}$, there exists $m \geqslant n$ so that, for every $\varepsilon>0$, there is some $\bar{v} \in \bar{V}$ such that $v_{m}(x) \leqslant \varepsilon v_{n}(x)$ whenever $\bar{v}(x)<v_{m}(x)$.

Proof. Assuming $\mathcal{V}$ satisfies condition (wV) and given $n \in \mathbf{N}$, choose $m>n$ as in (wV) and fix $\varepsilon>0$ and $k \geqslant m$. By (wV), we can find $\bar{v} \in \bar{V}$ such that $\bar{v}(x)<v_{m}(x)$ implies $v_{m}(x) \leqslant \varepsilon v_{n}(x) / 2$; thus $\bar{v}(x) \geqslant v_{m}(x)$ holds whenever $v_{m}(x) \geqslant \varepsilon v_{n}(x)$. Now $\bar{v} \leqslant \alpha_{k} v_{k}$ for some $\alpha_{k}>0$, and we may take $\delta(k, \varepsilon)=\varepsilon / \alpha_{k}$ because then

$$
v_{m}(x) \geqslant \varepsilon v_{n}(x) \text { implies } v_{k}(x) \geqslant \frac{1}{\alpha_{k}} \bar{v}(x) \geqslant \frac{1}{\alpha_{k}} v_{m}(x) \geqslant \delta(k, \varepsilon) v_{n}(x) .
$$

Conversely, let $\mathcal{V}$ be regularly decreasing. Given $n \in \mathbf{N}$, choose $m>n$ as in Definition 2.1, and fix $\varepsilon>0$; for each $k>m$, take $\delta(k, \varepsilon)>0$ so that

$$
v_{k}(x) \geqslant \delta(k, \varepsilon) v_{n}(x) \text { whenever } v_{m}(x) \geqslant \varepsilon v_{n}(x) .
$$

Taking

$$
\bar{v}=\inf \left\{\frac{1}{\varepsilon} v_{1}, \ldots, \frac{1}{\varepsilon} v_{m}, \frac{1}{\delta(m+1, \varepsilon)} v_{m+1}, \frac{1}{\delta(m+2, \varepsilon)} v_{m+2}, \ldots\right\} \in \check{V} \subset \bar{V},
$$

if $v_{m}(x)>\varepsilon v_{n}(x)$, then clearly

$$
\bar{v}(x)=\inf \left\{\frac{1}{\varepsilon} v_{m}(x), \frac{1}{\delta(m+1, \varepsilon)} v_{m+1}(x), \frac{1}{\delta(m+2, \varepsilon)} v_{m+2}(x), \ldots\right\} \geqslant v_{n}(x) .
$$

Consequently, $\bar{v}(x)<v_{m}(x) \leqslant v_{n}(x)$ implies $v_{m}(x) \leqslant \varepsilon v_{n}(x)$.

Condition (wV) was studied in the appendix of [7] (also see 0.4(c)); it is weaker than condition (V). Furthermore, if there exists $m \in \mathbf{N}$ such that $v_{m} \in \bar{V}$ (which holds, for instance, when $\mathscr{V}=\left\{\lambda_{n} v\right\}_{n \in N}$, where $\left(\lambda_{n}\right)_{n}$ is a decreasing sequence of positive numbers and $v$ is a fixed weight on $X$ ), then $\mathcal{V}$ also satisfies (wV).

The concept of being regularly decreasing is defined intrinsically (i.e., only in terms of the original sequence $\left.\mathfrak{V}=\left\{v_{n}\right\}_{n \in N}\right)$, and hence is preferable to condition (wV) where the definition involves the associated system $\bar{V}=\bar{V}_{\Im}$.

In fact, for the inductive system $\left\{C v_{n}(X, E)\right\}_{n \in \mathrm{N}}$, where $E$, say, is a normed linear space, given $n \in \mathbf{N}$, if $m>n$ is chosen as in condition (wV), we would know from [7, Appendix, Satz 1.6'] that $C v_{m}(X, E), \mathcal{V} C(X, E)$, and $C \bar{V}(X, E)$ induce the same topology on each bounded subset of $C v_{n}(X, E)$; if $m \geqslant n$ is chosen as in Definition 2.1 , an analogous direct argument would only show that the topologies of all spaces $C v_{k}(X, E), k=m, m+1, m+2, \ldots$, coincide on each bounded subset of $C v_{n}(X, E)$. However, using an abstract result of Neus [25, Satz 4], we already get as a consequence of this last property that $\mathcal{V} C(X, E)$ induces the same topology on each bounded set in $C v_{n}(X, E)$ as does each of the spaces $C v_{k}(X, E), k \geqslant m$. Thus, the additional information which we obtain from the equivalence of (wV) and regularly decreasing is really just that $C \bar{V}(X, E)$ does the same.

Proposition 2.2 and the subsequent remarks clearly demonstrate that our next theorem is an essential improvement over Theorem 1.3(d). 
2.3. TheOREM. Let $X$ denote a locally compact space, $(E,\|\cdot\|)$ a Banach space, and $\mathcal{V}=\left\{v_{n}\right\}_{n}$ a decreasing sequence of weights $v_{n}$ on $X$ such that $\inf \left\{v_{n}(x) ; x \in K\right\}>0$ for each compact subset $K$ of $X$ and $n=1,2, \ldots$ If $\mathcal{V}$ is regularly decreasing, $\mathcal{V}_{0} C(X, E)$ is complete and hence

$$
\mho_{0} C(X, E)=C \bar{V}_{0}(X, E)
$$

algebraically and topologically whereby $C \bar{V}_{0}(X, E)$ is again an ultrabornological (DF)-space.

Proof. (i) We will let $\tau$ denote the weighted topology on $C \bar{V}_{0}(X, E)$, and claim that it suffices to establish the following condition which we shall term (*):

For each $n \in \mathbf{N}$, let $m \geqslant n$ be chosen according to the definition of a regularly decreasing system. Then the closure $\bar{B}_{n}{ }^{\tau}$ of

$$
B_{n}=\left\{f \in C\left(v_{n}\right)_{0}(X, E) ; q_{v_{n}}(f)=\sup _{x \in X} v_{n}(x)\|f(x)\| \leqslant 1\right\}
$$

in $C \bar{V}_{0}(X, E)$ is contained in $B_{m}=\left\{f \in C\left(v_{m}\right)_{0}(X, E) ; q_{v_{m}}(f) \leqslant 1\right\}$.

Indeed, in view of Theorem 1.3, an obvious application of Raikov's completeness criterion for 1.c. spaces $([\mathbf{2 8}]$ or $[\mathbf{1 3}, 4.1$ (also see the proof of 7.4$)]$ ) will then establish our theorem.

(ii) To prove (*), fix $n \in \mathbf{N}$, and let $f$ be an arbitrary element of $\bar{B}_{n}{ }^{\tau}$. Firstly, we observe that $q_{v_{n}}(f) \leqslant 1$; indeed, the weighted topology of $C \bar{V}_{0}(X, E)$ is obviously stronger than the topology of pointwise convergence on $X$ (and is even finer than $c o$ ), and the pointwise closure of $\left\{g \in C v_{n}(X, E) ; q_{v_{n}}(g) \leqslant 1\right\}$ contains only functions $f$ which satisfy $q_{v_{n}}(f) \leqslant 1$ (cf. [7, 1.7]).

Next, taking $m \geqslant n$ as in Definition 2.1, we claim that $f \in C\left(v_{m}\right)_{0}(X, E)$. To prove this, let $\varepsilon>0$ and put $F=\left\{x \in X ; v_{m}(x)\|f(x)\| \geqslant \varepsilon\right\}$; we would show that $F$ is (relatively) compact. To see this, setting

$$
\alpha_{k}=\left\{\begin{array}{ll}
\frac{8}{\varepsilon}, & k<m \\
\frac{1}{\delta(k, \varepsilon / 8)}, & k \geqslant m
\end{array}\right\}, \quad k=1,2, \ldots,
$$

we put $\bar{v}=\inf \left\{\alpha_{k} v_{k} ; k \in \mathbf{N}\right\}$. Then $\bar{v} \in \check{V} \subset \bar{V}$, and so there exists $g \in B_{n}$ such that $q_{v}(f-g)<\varepsilon / 4$; note that also $G=\left\{x \in X ; v_{m}(x)\|g(x)\| \geqslant \varepsilon / 2\right\}$ is compact. We now observe that if $v_{m}(y)>\bar{v}(y)+\varepsilon v_{n}(y) / 8$ for some $y \in X$, then $k \geqslant m$ implies

$$
\alpha_{k} v_{k}(y) \geqslant \alpha_{k} \delta(k, \varepsilon / 8) v_{n}(y)=v_{n}(y)
$$

while $k<m$ yields

$$
\alpha_{k} v_{k}(y) \geqslant \alpha_{k} v_{m}(y)>\alpha_{k} \frac{\varepsilon}{8} v_{n}(y)=v_{n}(y) ;
$$

i.e., $\bar{v}(y) \geqslant v_{n}(y)$. However, this implies $v_{m}(y)>v_{n}(y)(1+\varepsilon / 8)>v_{n}(y)$ which is clearly impossible, and so $v_{m}(x) \leqslant \bar{v}(x)+\varepsilon v_{n}(x) / 8$ for all $x \in X$. Finally, taking 
$x \in F$, we have

$$
\begin{aligned}
v_{m}(x)\|g(x)\| & \geqslant v_{m}(x)\|f(x)\|-v_{m}(x)\|f(x)-g(x)\| \\
& \geqslant \varepsilon-\left(\bar{v}(x)+\frac{\varepsilon}{8} v_{n}(x)\right)\|f(x)-g(x)\| \\
& \geqslant \varepsilon-\bar{v}(x)\|f(x)-g(x)\|-\frac{\varepsilon}{8} v_{n}(x)(\|f(x)\|+\|g(x)\|) \\
& \geqslant \varepsilon-\frac{\varepsilon}{4}-\frac{\varepsilon}{8}-\frac{\varepsilon}{8}=\frac{\varepsilon}{2} .
\end{aligned}
$$

Thus $F \subset G$ whereby the argument is complete.

Instead of using Raikov's criterion in part (i) above, we could as well argue in the following way. If $C$ is an arbitrary bounded subset of $\mathcal{V}_{0} C(X, E)$, then, by a result of Grothendieck on countable (Hausdorff) l.c. inductive limits of ( $D F)$-spaces (cf. [23, I, §29, 5.(4)]), there exist $n \in \mathbf{N}$ and a bounded subset $C_{n}$ of $C\left(v_{n}\right)_{0}(X, E)$ such that $C$ is contained in the closure of $C_{n}$ in $\mathscr{V}_{0} C(X, E)$. A first application of condition (*) now proves that $\mathfrak{V}_{0} C(X, E)=$ ind $_{n \rightarrow} C\left(v_{n}\right)_{0}(X, E)$ is a regular inductive limit. At this point, let us use another result of Grothendieck (cf. [23, I, §29, 5.(3)]) whereby quasicompleteness and completeness are equivalent properties in $(D F)$-spaces. This then reduces the proof of Theorem 2.3 to showing that the closure of $B_{n}$ in $\mathcal{V}_{0} C(X, E)$ is complete for each $n \in \mathbf{N}$. But, again, from $(*)$ and Theorem 1.3, this last assertion is clear.

We can also give a different proof of 2.3 which we will include at this point since it yields additional insight. By $2.2, \mathcal{V}$ being regularly decreasing means that condition (wV) holds. Hence, from [7, Appendix, Satz 1.6' and p. 219] (see also 0.4(b) and (c)), we can infer that $\mathscr{T} C(X, E)=$ ind $_{n \rightarrow} C v_{n}(X, E)$ is (strongly) boundedly retractive for each normed space $E$ and complete whenever $E$ is a Banach space. Similarly, given $n \in \mathbf{N}$, if $m>n$ is chosen as in (wV), then the topologies of $C\left(v_{m}\right)_{0}(X, E)$, $\Upsilon_{0} C(X, E)$, and $C \bar{V}_{0}(X, E)$ all coincide on each bounded subset of $C\left(v_{n}\right)_{0}(X, E)$. If $E$ is a Banach space, an easy application of Grothendieck's result on bounded subsets in countable inductive limits of $(D F)$-spaces now implies the regularity of $\mho_{0} C(X, E)$ (see the remark at the end of 0.3$)$. Hence $\Upsilon_{0} C(X, E)=$ ind $_{n \rightarrow} C\left(v_{n}\right)_{0}(X, E)$ is (strongly) boundedly retractive and thereby also complete.

In fact, as we have pointed out above, condition (*), which was verified in part (ii) of the proof of Theorem 2.3, implies that $\mathcal{V}_{0} C(X, E)$ is regular so that the completeness of $E$ is actually not needed for this point; i.e., $\mathcal{V}_{0} C(X, E)$ is (strongly) boundedly retractive whenever $\mathcal{V}$ is regularly decreasing and $E$ is normed.

Here, we should remark that, using tensor product methods (essentially due to Hollstein [20]), Theorem 2.3 can be extended to include countable compactly regular inductive limits $E=$ ind $_{n \rightarrow} E_{n}$ of Banach spaces, at least when $X$ is $\sigma$-compact and $\mathcal{V}$ is a regularly decreasing sequence of strictly positive continuous weights $v_{n}$ on $X$; for further details, see Corollary 3.3 and the remark which follows it.

2.4. EXAMPLE. (a) For the sequence $\mathcal{V}=\left\{v_{n}\right\}_{n \in \mathrm{N}}$ of continuous weights $v_{n}$ on the open unit disk which was described in 1.5 , we know that the space $\mathcal{V}_{0} C(X)$ is not 
even sequentially complete. So, by Theorem 2.3 , the sequence $\mathcal{V}$ cannot be regularly decreasing, a fact which is also easily checked directly.

(b) Put $X=\mathbf{R}_{+} \backslash\{n \pi ; n=0,1,2, \ldots\}$ and define $\mathcal{V}=\left\{v_{n}\right\}_{n}$ by setting

$$
v_{n}(x)=\left\{\begin{array}{ll}
\frac{|\sin x|}{n}, & x \leqslant n \pi+\frac{\pi}{2} \\
\frac{1}{n}, & x>n \pi+\frac{\pi}{2}
\end{array}\right\}, \quad n=1,2, \ldots
$$

Then this sequence $\mathcal{V}$ of continuous weights also clearly fails to be regularly decreasing.

(c) Similarly, the sequence $\mathfrak{V}$ of weights on $\mathbf{N} \times \mathbf{N}$ in Köthe's example (Example 1.12 ) is not regularly decreasing.

2.5. TheOREM. Let $X$ denote a locally compact Hausdorff space and take $\mathcal{V}=\left\{v_{n}\right\}_{n}$ to be a decreasing sequence of strictly positive continuous weights $v_{n}$ on $X, n=1,2, \ldots$. If $\mathfrak{V}$ is not regularly decreasing, then $\mathfrak{V}_{0} C(X) \neq C \bar{V}_{0}(X)$.

Proof. (i) Assuming that $\mathfrak{V}$ is not regularly decreasing, we can fix $n \in \mathbf{N}$ so that, for every $m \geqslant n$, there exist $k_{m}>m$ and $\varepsilon_{m}>0$ for which, given $\delta>0$, it is always possible to find $x_{\delta} \in X$ such that

$$
v_{m}\left(x_{\delta}\right) \geqslant \varepsilon_{m} v_{n}\left(x_{\delta}\right) \quad \text { while } v_{k_{m}}\left(x_{\delta}\right)<\delta v_{n}\left(x_{\delta}\right)
$$

there is clearly no loss of generality in assuming that $\left\{\varepsilon_{m}\right\}_{m=n}^{\infty}$ is decreasing and $\left\{k_{m}\right\}_{n}^{\infty}$ is an increasing sequence.

For notational purposes, we define $\varphi: \mathbf{N} \rightarrow \mathbf{N}$ inductively by $\varphi(1)=n+1$ and $\varphi(i)=k_{\varphi(i-1)}+1, i=2,3, \ldots$; we shall write $k_{i}$ and $\varepsilon_{i}$ instead of $k_{\varphi(i)}$ and $\varepsilon_{\varphi(i)}$, respectively, $i=1,2, \ldots$ For each $i \in \mathbf{N}$, we will construct a sequence $\left\{\left(\delta_{i j}, x_{i j}, U_{i j}\right)\right\}_{j=1}^{\infty}$ for which the following conditions are satisfied for all $j \in \mathbf{N}$ :

(1) $0<\delta_{i j} \leqslant 1 / j$,

(2) $v_{\varphi(i)}\left(x_{i j}\right) \geqslant \varepsilon_{i} v_{n}\left(x_{i j}\right)$ while $v_{k_{i}}\left(x_{i j}\right)<\delta_{i j} v_{n}\left(x_{i j}\right)$,

(3) $U_{i j}$ is a compact neighborhood of $x_{i j}$ for which $x \in U_{i j}$ implies $v_{k_{i}}(x)<$ $\delta_{i j} v_{n}(x)$. Moreover, $U_{i j} \cap U_{r s}=\varnothing$ if either $i \neq r$ or $i=r$ but $j \neq s$, and $\cup_{j=1}^{\infty} U_{i j}$ is closed, as is $\bigcup\left\{U_{i j} ; j=1,2, \ldots, j \neq j_{0}\right\}$ for arbitrary $j_{0} \in \mathbf{N}$.

To start, considering $i=1$, put $\delta_{11}=1$ and choose $x_{11} \in X$ so that

$$
v_{\varphi(1)}\left(x_{11}\right) \geqslant \varepsilon_{1} v_{n}\left(x_{11}\right) \text { while } v_{k_{1}}\left(x_{11}\right)<\delta_{11} v_{n}\left(x_{11}\right) \text {. }
$$

Since $X$ is locally compact and $v_{k_{1}} / v_{n}$ is continuous on $X$, there is a compact neighborhood $U_{11}$ of $x_{11}$ such that $x \in U_{11}$ implies $v_{k_{1}}(x)<\delta_{11} v_{n}(x)$. Assume that $\left\{\left(\delta_{1 j}, x_{1 j}, U_{1 j}\right)\right\}_{j=1}^{p-1}$ have been chosen so that the applicable parts of (1)-(3) all hold. Let

$$
\beta_{1 p}=\inf \left\{\frac{v_{k_{1}}(x)}{v_{n}(x)} ; x \in \bigcup_{j=1}^{p-1} U_{1 j}\right\},
$$

and then choose $\delta_{1 p}<\min \left\{\beta_{1 p}, 1 / p\right\}$. Take $x_{1 p} \in X$ so that

$$
v_{\varphi(1)}\left(x_{1 p}\right) \geqslant \varepsilon_{1} v_{n}\left(x_{1 p}\right) \text { and } v_{k_{1}}\left(x_{1 p}\right)<\delta_{1 p} v_{n}\left(x_{1 p}\right) \text {. }
$$


Since $x_{1 p} \notin \cup_{j=1}^{p-1} U_{1 j}$, we can find a compact neighborhood $U_{1 p}$ of $x_{1 p}$ such that $x \in U_{1 p}$ implies $v_{k_{1}}(x)<\delta_{1 p} v_{n}(x)$ and $U_{1 p} \cap U_{1 j}=\varnothing$ if $j<p$. Inductively, therefore, we can obtain a sequence $\left\{\left(\delta_{1 j}, x_{1 j}, U_{1 j}\right)\right\}_{j=1}^{\infty}$ with the desired properties because, if $y \in \overline{\cup_{j=1}^{\infty} U_{1 j}} \backslash \cup_{j=1}^{\infty} U_{1 j}$, then it would follow that each neighborhood of $y$ contains points which belong to $U_{1 j}$ for indices $j$ larger than any given integer $j_{0}$. Hence $\delta_{1 j} \rightarrow 0$ for $j \rightarrow \infty$ would imply $v_{k_{1}}(y) / v_{n}(y)=0$ which is impossible. The same argument works for $\bigcup\left\{U_{1 j} ; j=1,2, \ldots, j \neq j_{0}\right\}, j_{0} \in \mathbf{N}$ arbitrary.

Assume now that sequences $\left\{\left(\delta_{i j}, x_{i j}, U_{i j}\right)\right\}_{j=1}^{\infty}$ have been selected for $i=1, \ldots$, $p-1$ so that (1), (2), (3) hold. To start the selection of the sequence for $i=p$, we first observe that, since $\delta_{i j} \leqslant 1 / j$, there exists an index $j_{0}=j_{0}(p)$ such that $\delta_{i j}<\varepsilon_{p}$ for all $j>j_{0}, i=1, \ldots, p-1$. Let

$$
\beta_{p 1}=\inf \left\{\frac{v_{k_{p}}(x)}{v_{n}(x)} ; x \in \bigcup_{i=1}^{p-1} \bigcup_{j=1}^{j_{0}} U_{i j}\right\},
$$

and then choose $\delta_{p 1}<\min \left\{\beta_{p 1}, 1\right\}$. Take $x_{p 1} \in X$ so that

$$
v_{\varphi(p)}\left(x_{p 1}\right) \geqslant \varepsilon_{p} v_{n}\left(x_{p 1}\right) \text { and } v_{k_{p}}\left(x_{p 1}\right)<\delta_{p 1} v_{n}\left(x_{p 1}\right) \text {. }
$$

Obviously $x_{p 1} \notin \cup_{i=1}^{p-1} \cup_{j=1}^{j_{0}} U_{i j}$, but, since any element $y \in U_{i j}, i=1, \ldots, p-1$, $j>j_{0}$, satisfies

$$
v_{\varphi(p)}(y) \leqslant v_{k_{i}}(y)<\delta_{i j} v_{n}(y) \leqslant \varepsilon_{p} v_{n}(y),
$$

we must also have $x_{p 1} \notin \cup_{i=1}^{p-1} \cup_{j=1}^{\infty} U_{i j}$. Since this last set is closed, we can find a compact neighborhood $U_{p 1}$ of $x_{p 1}$ such that $x \in U_{p 1}$ implies $v_{k_{p}}(x)<\delta_{p 1} v_{n}(x)$ and $U_{p 1} \cap U_{i j}=\varnothing$ if $i<p$ and $j=1,2, \ldots$.

Continuing inductively, it follows that a sequence $\left\{\left(\delta_{p j}, x_{p j}, U_{p j}\right)\right\}_{j=1}^{\infty}$ can be constructed just as in the case $i=1$ (taking

$$
\beta_{p q}=\inf \left\{\frac{v_{k_{p}}(x)}{v_{n}(x)} ; x \in\left(\bigcup_{i=1}^{p-1} \bigcup_{j=1}^{j_{0}} U_{i j}\right) \cup\left(\bigcup_{j=1}^{q-1} U_{p j}\right)\right\}
$$

and $\left.\delta_{p q}<\min \left\{\beta_{p q}, 1 / q\right\}\right)$ so that (1), (2), and (3) are satisfied for $i=1, \ldots, p$ (because also $\delta_{p j} \rightarrow 0$ as $j \rightarrow \infty$ ). Another application of finite induction leads to the desired sequence of sequences.

(ii) Taking $(i, j) \in \mathbf{N} \times \mathbf{N}$, choose a continuous function $\varphi_{i j}$ with $0 \leqslant \varphi_{i j} \leqslant 1$, $\varphi_{i j}\left(x_{i j}\right)=1$, and $\operatorname{supp}\left(\varphi_{i j}\right) \subset U_{i j}$. For each $i \in \mathbf{N}$, put $g_{i}=\sum_{j=1}^{\infty} \varphi_{i j}$; clearly, $g_{i}$ is a well-defined continuous function on $X, 0 \leqslant g_{i} \leqslant 1$, and $g_{i} \equiv 0$ on the complement of $\cup_{j=1}^{\infty} U_{i j}$. Now choose a decreasing sequence $\left\{\eta_{i}\right\}_{i \in \mathbf{N}}$ with $\eta_{i}>0$ and $\lim _{i \rightarrow \infty} \eta_{i}=0$, and set $h_{i}=\eta_{i} g_{i} / 2^{i}, i=1,2, \ldots$; then $\left\{h_{i}\right\}_{i \in \mathrm{N}}$ is a sequence of nonnegative continuous bounded functions on $X$. We observe that, given $\varepsilon>0$, we can choose $p \in \mathbf{N}$ so that $\eta_{p}<\varepsilon$ and hence, uniformly on $X$,

$$
\sum_{i=p}^{\infty} h_{i}=\sum_{i=p}^{\infty} \eta_{i} \frac{1}{2^{i}} g_{i} \leqslant \eta_{p} \sum_{i=p}^{\infty} \frac{1}{2^{i}} \leqslant \eta_{p}<\varepsilon .
$$

Thus $h=\sum_{i=1}^{\infty} h_{i} \geqslant 0$ is a well-defined bounded continuous function on $X$ which vanishes on the complement of $\cup_{i=1}^{\infty} \cup_{j=1}^{\infty} U_{i j}$. 
We claim that the continuous function $f=h / v_{n}$ belongs to $C \bar{V}_{0}(X) \backslash \mathcal{V}_{0} C(X)$. Fixing $i \in \mathbf{N}$,

$$
\begin{aligned}
v_{\varphi(i)}\left(x_{i j}\right) f\left(x_{i j}\right) & =\frac{v_{\varphi(i)}\left(x_{i j}\right)}{v_{n}\left(x_{i j}\right)} h\left(x_{i j}\right) \geqslant \varepsilon_{i} h\left(x_{i j}\right) \geqslant \varepsilon_{i} h_{i}\left(x_{i j}\right)=\frac{\varepsilon_{i} \eta_{i}}{2^{i}} g_{i}\left(x_{i j}\right) \\
& \geqslant \frac{\varepsilon_{i} \eta_{i}}{2^{i}}, \quad j=1,2, \ldots
\end{aligned}
$$

But this implies that

$$
\left\{x_{i j}\right\}_{j=1}^{\infty} \subset F_{i}=\left\{x \in X ; v_{\varphi(i)}(x) f(x) \geqslant \frac{\varepsilon_{i} \eta_{i}}{2^{i}}\right\}
$$

whereby $F_{i}$ is not compact since $v_{k_{i}} / v_{n}$ must necessarily vanish at any cluster point of $\left\{x_{i j}\right\}_{j=1}^{\infty}$ because $\lim _{j \rightarrow \infty} \delta_{i j}=0$. We can thus conclude that $f \notin C\left(v_{\varphi(i)}\right)_{0}(X)$; i.e., since $i \in \mathbf{N}$ was arbitrary and $\varphi(i)$ tends to $\infty$ with $i, f \notin \mathfrak{V}_{0} C(X)$. Finally, take $v \in \bar{V}$ and let $\varepsilon>0$. Then $v \leqslant \inf \left\{\alpha_{k} v_{k} ; k \in \mathbf{N}\right\}$ where $\alpha_{k}>0, k=1,2, \ldots$; put

$$
F=\{x \in X ; v(x) f(x) \geqslant \varepsilon\} .
$$

Since $f$ vanishes on the complement of $\cup_{i=1}^{\infty} \cup_{j=1}^{\infty} U_{i j}$, any element of $F$ belongs to some $U_{i j}$. If $x \in U_{i j}$ where $\eta_{i} / 2^{i}<\varepsilon / \alpha_{n}$, then

$$
v(x) f(x) \leqslant \alpha_{n} v_{n}(x) f(x)=\alpha_{n} h(x)=\alpha_{n} h_{i}(x)=\alpha_{n} \frac{\eta_{i}}{2^{i}} g_{i}(x) \leqslant \alpha_{n} \frac{\eta_{i}}{2^{i}}<\varepsilon .
$$

If $x \in U_{i j}$ where $\delta_{i j}<2^{i} \varepsilon / \eta_{i} \alpha_{k_{i}}$, then

$$
v(x) f(x) \leqslant \alpha_{k_{i}} v_{k_{i}}(x) f(x)=\alpha_{k_{i}} \frac{v_{k_{i}}(x)}{v_{n}(x)} h(x) \leqslant \alpha_{k_{i}} \delta_{i j} h(x) \leqslant \alpha_{k_{i}} \delta_{i j} \frac{\eta_{i}}{2^{i}}<\varepsilon
$$

i.e., again $x \notin F$. Consequently, since $\eta_{i} / 2^{i} \rightarrow 0$ as $i \rightarrow \infty$ and $\delta_{i j} \rightarrow 0$ as $j \rightarrow \infty$, $i=1,2, \ldots, F$ is contained in a finite union of the sets $U_{i j}$ whereby $F$ is compact. The proof is thus complete.

We will now show that, if the sequence $\mathscr{V}=\left\{v_{n}\right\}_{n}$ in 2.5 is not regularly decreasing, then, in fact, $\mathcal{V}_{0} C(X)=$ ind $_{n \rightarrow} C\left(v_{n}\right)_{0}(X)$ cannot be a regular inductive limit. To this purpose, let the functions $\varphi_{i j}, g_{i}$, and $h_{i}, i, j=1,2, \ldots$, be constructed as in the proof of Theorem 2.5 and define $f_{i}=h_{i} / v_{n}$ for every $i \in \mathbf{N}$. We claim that

$$
f_{i} \in C\left(v_{k_{i}}\right)_{0}(X) \backslash C\left(v_{\varphi(i)}\right)_{0}(X) \text {. }
$$

Fix $\varepsilon>0$. By definition, $f_{i}$ vanishes on the complement of $\cup_{j=1}^{\infty} U_{i j}$. Since $\delta_{i j} \rightarrow 0$ as $j \rightarrow \infty$, we can find $j_{1}=j_{1}(\varepsilon)$ such that $\eta_{i} \delta_{i j} / 2^{i}<\varepsilon$ for all $j>j_{1}$. Hence $x \in U_{i j}$, $j>j_{1}$, implies

$$
v_{k_{i}}(x) f_{i}(x)=\frac{v_{k_{i}}(x)}{v_{n}(x)} h_{i}(x) \leqslant \delta_{i j} \frac{\eta_{i}}{2^{i}} g_{i}(x) \leqslant \delta_{i j} \frac{\eta_{i}}{2^{i}}<\varepsilon,
$$

and therefore $\left\{x \in X ; v_{k_{i}}(x) f_{i}(x) \geqslant \varepsilon\right\} \subset \cup_{j=1}^{j_{1}} U_{i j}$ is compact. This proves $f_{i} \in$ $C\left(v_{k_{i}}\right)_{0}(X)$. An inspection of the corresponding part of the proof of Theorem 2.5 reveals that $f_{i} \notin C\left(v_{\varphi(i)}\right)_{0}(X)$ since

$$
v_{\varphi(i)}\left(x_{i j}\right) f_{i}\left(x_{i j}\right)=\frac{v_{\varphi(i)}\left(x_{i j}\right)}{v_{n}\left(x_{i j}\right)} h_{i}\left(x_{i j}\right) \geqslant \varepsilon_{i} h_{i}\left(x_{i j}\right) \geqslant \frac{\varepsilon_{i} \eta_{i}}{2^{i}}
$$


$j=1,2, \ldots$, whence $F_{i}=\left\{x \in X ; v_{\varphi(i)}(x) f_{i}(x) \geqslant \varepsilon_{i} \eta_{i} / 2^{i}\right\}$, since it contains $\left\{x_{i j}\right\}_{j=1}^{\infty}$, cannot be compact.

Now, $B=\left\{f_{i} ; i \in \mathbf{N}\right\}$ is a subset of $\mathscr{V}_{0} C(X)$ which, since $\varphi(i) \rightarrow \infty$ as $i \rightarrow \infty$, is not contained in $C\left(v_{l}\right)_{0}(X)$ for $l=1,2, \ldots$. But for $f=\sum_{i=1}^{\infty} f_{i}=\left(v_{n}\right)^{-1} \sum_{i=1}^{\infty} h_{i}$, the proof of Theorem 2.5 yields that $f \in C \bar{V}_{0}(X)$; since $f_{i} \leqslant f, i=1,2, \ldots, B$ is clearly bounded in $C \bar{V}_{0}(X)$. From Theorem 1.3(a), we can now conclude that $B$ is also a bounded subset of $\mathfrak{V}_{0} C(X)$, and so $B$ provides the desired counterexample to regularity of $\mathfrak{T}_{0} C(X)$.

We should add that the nonregularity of $\mathfrak{T}_{0} C(X)$ for a decreasing, but not regularly decreasing, sequence $\mathfrak{V}=\left\{v_{n}\right\}_{n \in \mathbf{N}}$ of strictly positive continuous weights on $X$ is always due to the fact that there exists a bounded sequence $B$ in $\mathfrak{V}_{0} C(X)$ which is not contained in any $C\left(v_{n}\right)_{0}(X), n=1,2, \ldots$, as can be seen from the preceding proof. Hence, in this case, $\mathfrak{\mho}_{0} C(X)$ is not even $\alpha$-regular in the terminology of $[13,5.2]$. Indeed, regularity fails here only in this way since, using the regularity of $\mathscr{T} C(X, E)=\operatorname{ind}_{n \rightarrow} C v_{n}(X, E)$ (established in [7, 1.7]), the following is obvious:

If $E$ is a normed space and $\mathfrak{V}=\left\{v_{n}\right\}_{n \in \mathbf{N}}$ is a decreasing sequence of strictly positive weights $v_{n}$ on the completely regular Hausdorff space $X, \mathfrak{V}_{0} C(X, E)=$ ind $_{n \rightarrow} C\left(v_{n}\right)_{0}(X, E)$ is always $\beta$-regular; i.e., given a set $B$ contained in some $C\left(v_{n}\right)_{0}(X, E)$ and bounded with respect to the inductive limit topology of $\mathfrak{V}_{0} C(X, E)$, there exists $m=m(B) \geqslant n$ such that $B$ is bounded with respect to the norm topology of $C\left(v_{m}\right)_{0}(X, E)$.

Let us sum up as follows:

2.6. THEOREM. Let $X$ denote a locally compact space and $\mathfrak{V}=\left\{v_{n}\right\}_{n}$ a decreasing sequence of strictly positive continuous weights on $X$. Then

(a) $\mathfrak{V}$ is regularly decreasing $\Leftrightarrow \mathfrak{V}_{0} C(X, E)$ is (strongly) boundedly retractive for each normed space $E \Leftrightarrow \mathfrak{V}_{0} C(X, E)$ is complete for each Banach space $E \Leftrightarrow$ $\mho_{0} C(X)$ is complete $\Leftrightarrow \mho_{0} C(X)=C \bar{V}_{0}(X)$

(b) $\mathfrak{V}$ is not regularly decreasing $\Leftrightarrow \mathfrak{V}_{0} C(X)=\operatorname{ind}_{n \rightarrow} C\left(v_{n}\right)_{0}(X)$ is not $\alpha$-regular $\Leftrightarrow \mathfrak{V}_{0} C(X)$ is not regular $\Leftrightarrow \mathfrak{V}_{0} C(X)$ is not (sequentially) complete $\Leftrightarrow$ $\widetilde{V}_{0} C(X) \underset{\neq}{\subset} C \bar{V}_{0}(X)$.

If $\mathscr{V}$ satisfies (wV), then it was proved in [7, Appendix] that $\mathfrak{V} C(X, E)$ is (strongly) boundedly retractive for each normed space $E$. Conversely, if $\widetilde{V} C(X)$ is boundedly retractive, it is easy to see (along the lines of the second proof of Theorem 2.3) that $\mathfrak{V}_{0} C(X)$ must also be boundedly retractive and hence complete. Theorem 2.6 thus allows us to likewise formulate equivalences in terms of $\mathcal{} \mathcal{} C(X)$.

2.7. Corollary. Under the hypotheses of Theorem 2.6, $\mathfrak{V}$ is regularly decreasing $\Leftrightarrow \widetilde{ } C(X)=\operatorname{ind}_{n \rightarrow} C v_{n}(X)$ is boundedly retractive $\Leftrightarrow \mathcal{V} C(X, E)$ is (strongly) boundedly retractive for each normed space $E$.

Among other things, of course, Theorem 2.6 provides a ready source for examples of $(L B)$-spaces $\widetilde{V}_{0} C(X)$ which fail to be complete and, in fact, are not even $\alpha$-regular inductive limits. Also, Corollary 2.7 in conjunction with Theorem 1.13 yields new examples of complete $(L B)$-spaces $\mathfrak{T} A(X)$ which are not boundedly retractive. 
Finally, let us note that our regularly decreasing condition also describes the position of the subspace $\mathcal{V}_{0} C(X)$ in $\mathcal{V} C(X)$.

2.8. COROLLARY. Under the hypotheses of Theorem 2.6, the following statements are equivalent:

(1) $\mathfrak{T}$ is regularly decreasing;

(2) $\mathcal{V}_{0} C(X, E)$ is a closed subspace of $\mathscr{V} C(X, E)$ for each Banach space $E$;

(3) $\mathfrak{V}_{0} C(X)$ is a closed subspace of $\mathfrak{V} C(X)$;

(4) for each Banach space $E, \mathfrak{V}_{0} C(X, E) \cap C v_{n}(X, E)$ is closed in $C v_{n}(X, E)$, $n=1,2, \ldots$;

(5) $\mathfrak{V}_{0} C(X) \cap C v_{n}(X)$ is closed in $C v_{n}(X)$ for each $n \in \mathbf{N}$.

Proof. If $\mathscr{V}$ is regularly decreasing and $E$ is a Banach space, $\mathscr{V}_{0} C(X, E)$ is a complete topological linear subspace of $\mathfrak{V} C(X, E)$ by Corollary $1.4($ a) and Theorem 2.3 ; i.e., (1) implies (2). Since (2) clearly implies (4), while (2) $\Rightarrow(3)$, (4) $\Rightarrow(5)$, and (3) $\Rightarrow$ (5) are all trivial, it remains to show (5) $\Rightarrow$ (1).

If $\mathcal{V}$ is not regularly decreasing, returning to the proof of Theorem 2.5 and the subsequent remark on regularity of $\mathcal{V}_{0} C(X)$, we see that the functions $f_{i}=h_{i} / v_{n}$, $i=1,2, \ldots$, belong to $\Upsilon_{0} C(X) \cap C v_{n}(X)$. Now, obviously, $\sum_{i=1}^{N} f_{i}=\left(v_{n}\right)^{-1} \sum_{i=1}^{N} h_{i}$ converges to $f=h / v_{n}$ in $C v_{n}(X)$ as $N \rightarrow \infty$ whereby $\mathfrak{V}_{0} C(X) \cap C v_{n}(X)$ is not closed in $C v_{n}(X)$ since $h / v_{n} \notin \mathfrak{V}_{0} C(X)$.

3. Consequences for $\varepsilon$-tensor products. The foregoing development provides a tool for the investigation of $\varepsilon$-products and $\varepsilon$-tensor products of weighted inductive limit spaces, and we now proceed to illustrate the utility of our results by resolving several questions which remained open in [7]. For the general setting and related work, we shall refer to that article and 4.4 below.

Let us first recall some notation. The e-product $E \varepsilon F$ of two l.c. spaces $E$ and $F$ is defined to be the space $\mathcal{L}_{e}\left(F_{c}^{\prime}, E\right)$ of all continuous linear transformations from $F_{c}^{\prime}$ into $E$ under the topology of uniform convergence on the equicontinuous subsets of $F^{\prime}$, where $F_{c}^{\prime}$ denotes $F^{\prime}$ with the topology of uniform convergence on the absolutely convex compact subsets of $F$. (There is always a canonical topological isomorphism between $E \varepsilon F$ and $F \varepsilon E$.)

Now $E \otimes F$ can be embedded in $E \varepsilon F$ by putting

$$
\left(\sum_{j=1}^{n} e_{j} \otimes f_{j}\right)\left(f^{\prime}\right)=\sum_{j=1}^{n}\left\langle f_{j}, f^{\prime}\right\rangle e_{j}, \quad f^{\prime} \in F^{\prime},
$$

and we endow $E \otimes F$ with the topology thus induced by $E \varepsilon F$. The resulting 1.c. space is denoted by $E \otimes_{\varepsilon} F$, and its completion $E \check{\otimes}_{\varepsilon} F$ is called the (completed) $\varepsilon$-tensor product of $E$ and $F$. Properties of the $\varepsilon$-product and the $\varepsilon$-tensor product can be found in $[16,31]$, or $[23$, II].

3.1. THEOREM. Let $X$ be a locally compact Hausdorff space, E a l.c. space, and take $\mathcal{V}=\left\{V_{n}\right\}_{n}$ to be a sequence of systems of weights on $X$ with $V_{n+1} \leqslant V_{n}$ for each $n \in \mathbf{N}$.

(a) $\bigvee_{0} C(X) \otimes_{\varepsilon} E$ is a dense topological linear subspace of $C \bar{V}_{0}(X, E)$.

(b) $\mathscr{V}_{0} C(X) \check{\otimes}_{\varepsilon} E=C \bar{V}_{0}(X, E)$ if, and only if, $C \bar{V}_{0}(X, E)$ is complete. 
(c) If $\mathcal{K} \leqslant V_{n}, n=1,2, \ldots$, and $E$ is complete, then

$$
\mathfrak{V}_{0} C(X) \check{\otimes}_{\varepsilon} E=C \bar{V}_{0}(X) \check{\otimes}_{\varepsilon} E=C \bar{V}_{0}(X) \varepsilon E=C \bar{V}_{0}(X, E) \text {. }
$$

(d) The equation

$$
\left.\mathfrak{V}_{0} C \dot{(} X\right) \check{\otimes}_{\varepsilon} E=\mathfrak{V}_{0} C(X) \varepsilon E=\mathfrak{V}_{0} C(X, E)
$$

holds whenever $\mathcal{V}=\left\{v_{n}\right\}_{n \in \mathrm{N}}$ is a regularly decreasing sequence of weights on $X$ such that $\inf \left\{v_{n}(x) ; x \in K\right\}>0$ for each compact subset $K$ of $X, n=1,2, \ldots$, and $E$ is a Banach space; in this case, $\mathfrak{V}_{0} C(X) \check{\otimes}_{\varepsilon} E=\mathfrak{V}_{0} C(X) \varepsilon E$ is an ultrabornological $(D F)$ space.

Proof. (a) By 1.3(a), $\Upsilon_{0} C(X)$ is a topological subspace of $C \bar{V}_{0}(X)$. Since the operation $\otimes_{\varepsilon}$ preserves topological linear subspaces, $\Upsilon_{0} C(X) \otimes_{\varepsilon} E$ is a topological linear subspace of $C \bar{V}_{0}(X) \otimes_{\varepsilon} E$ which, in turn, forms a topological subspace of $C \bar{V}_{0}(X, E)$ by [3, I, 4.5.(1)]. Moreover, $\mathcal{V}_{0} C(X)$ is dense in $C \bar{V}_{0}(X)$, while $C \bar{V}_{0}(X) \otimes$ $E$ is dense in $C \bar{V}_{0}(X, E)$ by $[3, \mathrm{I}, 5.1]$. Hence, $\mathcal{V}_{0} C(X) \otimes E$ is also dense in $C \bar{V}_{0}(X, E)$.

(b) is immediate from (a).

(c) Under our assumptions, $C \bar{V}_{0}(X, E)$ is complete, and hence we have $\mathfrak{V}_{0} C(X)$ $\check{\otimes}_{\varepsilon} E=C \bar{V}_{0}(X, E)$ from (b). Because $F \check{\otimes}_{\varepsilon} G=\hat{F} \check{\otimes}_{\varepsilon} \hat{G}$ is valid for any two l.c. spaces $F, G$ and their respective completions $\hat{F}$ and $\hat{G}, \mathcal{V}_{0} C(X) \check{\otimes}_{\varepsilon} E=C \bar{V}_{0}(X) \hat{\otimes}_{\varepsilon} E$. Finally, $C \bar{V}_{0}(X)$ is a complete 1.c. space with a.p. (proof of $1.4(\mathrm{a})$, for example), and so $C \bar{V}_{0}(X) \check{\otimes}_{\varepsilon} E=C \bar{V}_{0}(X) \varepsilon E$ follows from the well-known fact (due to L. Schwartz [31]) that $F \ddot{\otimes}_{\varepsilon} G=F \varepsilon G$ holds for complete 1.c. spaces $F$ and $G$ if one of them has a.p.

(d) From what we have just said, 1.4(a) clearly implies $\mathfrak{V}_{0} C(X) \hat{\otimes}_{\varepsilon} E=\mathfrak{V}_{0} C(X) \varepsilon E$ whenever $\mathcal{V}_{0} C(X)$ and $E$ are complete. We can therefore apply (the scalar case of) 2.3 to get the first equality. But $\mathfrak{V}_{0} C(X) \check{\otimes}_{\varepsilon} E=\mathfrak{V}_{0} C(X, E)$ follows from (c) and (the vector valued case of) 2.3 .

Since Theorem 1.6 treats only the scalar case, the information on tensor products of weighted inductive limits of type $\Upsilon_{0} A(X)$ which follows from $\S 1$ is somewhat incomplete when compared with results like 3.1(d) for Banach space valued continuous functions, but something can still be said.

3.2. Corollary. Let $X$ be a locally compact Hausdorff space, take $E$ to be a quasi-complete l.c. space, and let $\mathfrak{V}=\left\{v_{n}\right\}_{n \in N}$ be a decreasing sequence of weights on $X$ such that $\inf \left\{v_{n}(x) ; x \in K\right\}>0$ for each compact subset $K$ of $X, n=1,2, \ldots$ Suppose, further, that condition (V) holds, $(A(X), \mathrm{co})$ is a semi-Montel subspace of $(C(X), \mathrm{co})$, and that

$$
A(X, E)=\left\{f \in C(X, E) ; e^{\prime} \circ f \in A(X) \text { for each } e^{\prime} \in E^{\prime}\right\} .
$$

(a) Then

$$
\Upsilon_{0} A(X) \varepsilon E=A \bar{V}_{0}(X) \varepsilon E=A \bar{V}_{0}(X, E),
$$

and this space equals $\mathfrak{V}_{0} A(X) \check{\otimes}_{\varepsilon} E$ whenever $E$ is complete and $\mathfrak{V}_{0} A(X)$ or $E$ has a.p.

(b) Moreover, if $E$ is a Banach space, then $\mathfrak{V}_{0} A(X) \varepsilon E=\mathfrak{V}_{0} A(X, E)$ algebraically and has the topology induced from $\mathcal{V}_{0} C(X, E)$. 
Proof. (a) We know from 1.6 that $\mathfrak{V}_{0} A(X)=A \bar{V}_{0}(X)$. Also, in the terminology of [5, II], $A(X, E)$ is "weakly determined" by $A(X)$, and hence we can conclude from [5, II, Theorem 2.9] that $\Upsilon_{0} A(X) \varepsilon E=A \bar{V}_{0}(X) \varepsilon E=A \bar{V}_{0}(X, E)$. (Holomorphic vector valued functions provide one instance where this "weakly determined" condition is satisfied; other examples can be found in $[5, \mathrm{II}]$.)

(b) The algebraic equality $\mathcal{V}_{0} A(X) \varepsilon E=\mathscr{V}_{0} A(X, E)$ for arbitrary complete ( $D F$ )-spaces $E$ was deduced in [7, 3.10 (also see the remark following 1.12)] from an abstract theorem on the $\varepsilon$-product. The topological result follows from 3.1(d) and the fact that the $\varepsilon$-product preserves topological linear subspaces.

At this point, we would like to add a few remarks on $\varepsilon$-products and $\varepsilon$-tensor products of the spaces $\mathscr{V}_{0} C(X)$ (and $\Upsilon_{0} A(X)$, respectively) with some (special) 1.c. inductive limit spaces $E$. In fact, we will use 3.1(d) to obtain an extension of [20, Proposition 5.6]. In that article, Hollstein applied an abstract theorem which permits commuting inductive limits and $\varepsilon$-tensor products, together with a preliminary version of our Theorem 3.1(d) (where $\mathfrak{V}$ was still assumed to satisfy condition (V)) to generalize the tensor product representation $\mathcal{V}_{0} C(X, E)=\mathcal{V}_{0} C(X) \check{\otimes}_{\varepsilon} E$ (in a special case) to compactly regular inductive limits $E$ of Banach spaces.

3.3. Corollary. $\mathcal{V}_{0} C(X, E)=\mathscr{V}_{0} C(X) \check{\otimes}_{\varepsilon} E$ still holds when $X$ is a locally compact Hausdorff space, $E=$ ind $_{n \rightarrow} E_{n}$ a compactly regular inductive limit of Banach spaces, and $\mathcal{V}=\left\{v_{n}\right\}_{n \in \mathbf{N}}$ is a regularly decreasing sequence of strictly positive continuous weights $v_{n}$ on $X$ such that the maximal system $\bar{V}$ associated with $\mathcal{V}$ is equivalent to $a$ system $\underline{V}$ of continuous weights on $X ;$ i.e., $\underline{V} \leqslant \bar{V}$ and $\bar{V} \leqslant \underline{V}$.

The last assumption is certainly valid whenever $X$ is additionally $\sigma$-compact, as can be seen from the proposition in $\$ 0.2$.

REMARK. It follows from the proof of 3.3 below that we also have the following topological isomorphisms (referring to $\S 0.2$ for the definition of $\mathscr{D} \mathcal{V}_{0} C(X, E)$ ):

$$
\begin{aligned}
& \mho_{0} C(X, E)=\mathfrak{\mho}_{0} C(X) \varepsilon E=\mathfrak{\mho}_{0} C(X) \check{\otimes}_{\varepsilon} E \\
& =C \bar{V}_{0}(X) \check{\otimes}_{\varepsilon} E=C \bar{V}_{0}(X) \varepsilon E=C \bar{V}_{0}(X, E) \\
& =\operatorname{ind}_{n \rightarrow} \mathscr{V}_{0} C\left(X, E_{n}\right)=\operatorname{ind}_{n \rightarrow}\left(\mathscr{V}_{0} C(X) \varepsilon E_{n}\right)=\operatorname{ind}_{n \rightarrow}\left(\mathscr{V}_{0} C(X) \check{\otimes}_{\varepsilon} E_{n}\right) \\
& =\operatorname{ind}_{m \rightarrow} C\left(v_{m}\right)_{0}(X, E)=\operatorname{ind}_{m \rightarrow}\left(C\left(v_{m}\right)_{0}(X) \varepsilon E\right)=\operatorname{ind}_{m \rightarrow}\left(C\left(v_{m}\right)_{0}(X) \check{\otimes}_{\varepsilon} E\right) \\
& =\operatorname{ind}_{m \rightarrow} \text { ind }\left(\text { or ind ind } \operatorname{ind}_{m \rightarrow}\right) C\left(v_{m}\right)_{0}\left(X, E_{n}\right) \\
& \left(\text { or } C\left(v_{m}\right)_{0}(X) \varepsilon E_{n} \text { or } C\left(v_{m}\right)_{0}(X) \check{\otimes}_{\varepsilon} E_{n}\right) \\
& =\mathscr{D V}_{0} C(X, E)=\operatorname{ind}_{n \rightarrow}\left(C\left(v_{n}\right)_{0}(X) \varepsilon E_{n}\right)=\operatorname{ind}_{n \rightarrow}\left(C\left(v_{n}\right)_{0}(X) \check{\otimes}_{\varepsilon} E_{n}\right) \text {. }
\end{aligned}
$$

In particular, for a $\sigma$-compact locally compact Hausdorff space $X$ and a regularly decreasing sequence $\mathcal{V}$ of strictly positive continuous weights $v_{n}$ on $X$, this extends the algebraic and topological isomorphism $\mathcal{V}_{0} C(X, E)=C \bar{V}_{0}(X, E)$ from the Banach space case (cf. Theorem 2.3) to compactly regular inductive limits $E=\operatorname{ind}_{n \rightarrow} E_{n}$ of Banach spaces, and $\mathfrak{V}_{0} C(X, E)=\mathfrak{V}_{0} C(X) \check{\otimes}_{\varepsilon} E$ is again an ultrabornological $(D F)$-space. 
The proof of 3.3 is based upon an analysis of Hollstein's argument [20, Proposition 5.6]. From [3, I, 5.3], we know that

$$
\begin{aligned}
\mathscr{V}_{0} C(X, E) & =\operatorname{ind}_{m \rightarrow} C\left(v_{m}\right)_{0}(X, E)=\operatorname{ind}_{m \rightarrow}\left(C\left(v_{m}\right)_{0}(X) \check{\otimes}_{\varepsilon} E\right) \\
& =\operatorname{ind}_{m \rightarrow}\left(C\left(v_{m}\right)_{0}(X) \check{\otimes}_{\varepsilon}\left(\operatorname{ind}_{n \rightarrow} E_{n}\right)\right) .
\end{aligned}
$$

Since $C\left(v_{m}\right)_{0}(X)$ is a $\varrho_{\infty}$-space, $m=1,2, \ldots$ (Hollstein [20, Proposition 2.3 and Corollary 2.1]), we may apply [20, Corollary 4.4$]$ to obtain

$$
\operatorname{ind}_{m \rightarrow}\left(C\left(v_{m}\right)_{0}(X) \check{\otimes}_{\varepsilon}\left(\operatorname{ind}_{n \rightarrow} E_{n}\right)\right)=\operatorname{ind}_{m \rightarrow} \operatorname{ind}\left(C\left(v_{m}\right)_{0}(X) \check{\otimes}_{\varepsilon} E_{n}\right) \text {. }
$$

At this point, invoking $[7,4.5]$ to continue,

$$
\begin{aligned}
\operatorname{ind} \operatorname{ind}\left(C\left(v_{m}\right)_{0}(X) \check{\otimes}_{\varepsilon} E_{n}\right) & =\underset{n \rightarrow}{\operatorname{ind}}\left(C\left(v_{n}\right)_{0}(X) \check{\otimes}_{\varepsilon} E_{n}\right) \\
& =\text { ind ind }\left(C\left(v_{m}\right)_{0}(X) \check{\otimes}_{\varepsilon} E_{n}\right) \\
& =\operatorname{ind}_{n \rightarrow} \operatorname{ind} C\left(v_{m}\right)_{0}\left(X, E_{n}\right)=\operatorname{ind}_{n \rightarrow} \mathscr{V}_{0} C\left(X, E_{n}\right) .
\end{aligned}
$$

Moreover, Theorem 3.1(d) implies

$$
\operatorname{ind}_{n \rightarrow} \mathscr{V}_{0} C\left(X, E_{n}\right)=\operatorname{ind}_{n \rightarrow}\left(\mathscr{V}_{0} C(X) \check{\otimes}_{\varepsilon} E_{n}\right) \text {. }
$$

Now, from Theorem 2.3 and our assumption on $\bar{V}$, we have $\mathfrak{V}_{0} C(X)=C \bar{V}_{0}(X)=$ $C \underline{V}_{0}(X)$ is a complete $(D F)$-space with a.p. (see Corollary 1.4(a)) and an $\varepsilon$-space in Hollstein's terminology [20, Proposition 2.3]. Hence, from [20, Theorem 4.1],

$$
\operatorname{ind}\left(\Upsilon_{n} C(X) \check{\otimes}_{\varepsilon} E_{n}\right)=\mathscr{V}_{0} C(X) \check{\otimes}_{\varepsilon}\left(\operatorname{ind}_{n \rightarrow} E_{n}\right)=\Upsilon_{0} C(X) \check{\otimes}_{\varepsilon} E .
$$

The remaining isomorphisms in $(*)$ are clear from 3.1 .

The topological isomorphism $\mathscr{V}_{0} A(X, E)=\mathfrak{V}_{0} A(X) \varepsilon E$ was also proved in [7, 4.8], but in a different way (using a factorization theorem for the algebraic equality and an open mapping theorem plus the fact that the $\varepsilon$-product of Silva spaces is a Silva space to show that the corresponding topologies agree), under the assumption that $X$ is a $k_{\mathbf{R}}$-space, $E$ is a Silva space, $\mathscr{V}=\left\{v_{n}\right\}_{n}$ is a decreasing sequence of weights on $X$ (such that $\inf \left\{v_{n}(x) ; x \in K\right\}>0$ for each compact subset $K$ of $X$ and each $n=1,2, \ldots)$ which satisfies condition $(\mathrm{V}),(A(X), \mathrm{co})$ is a semi-Montel subspace of $(C(X), \mathrm{co})$ and that

$$
A(X, E)=\left\{f \in C(X, E) ; e^{\prime} \circ f \in A(X) \text { for each } e^{\prime} \in E^{\prime}\right\} .
$$

Further, if $\mathfrak{V}_{0} A(X)$ (or $E$ ) is nuclear, it is enough to assume that $E$ is a complete bornological ( $D F)$-space instead of a Silva space (cf. [7, 4.7.(4)]), and then

$$
\Upsilon_{0} A(X, E)=\Upsilon_{0} A(X) \varepsilon E=\mathscr{V}_{0} A(X) \check{\otimes}_{\varepsilon} E=\Upsilon_{0} A(X) \hat{\otimes}_{\pi} E
$$

We now turn to $\varepsilon$-tensor products of two inductive limit spaces $\mathfrak{V}_{0} C(X)$ (resp. $\left.\Upsilon_{0} A(X)\right)$ 
3.4. Proposition. Let $X_{i}$ denote a locally compact Hausdorff space, and take $\mathfrak{V}_{i}=\left\{V_{n, i}\right\}_{n}$ to be a sequence of systems of weights on $X_{i}$ with $V_{n+1, i} \leqslant V_{n, i}$ for each $n \in \mathbf{N}, i=1,2$, and denote the system of weights $\left\{\bar{v}_{1} \otimes \bar{v}_{2} ; \bar{v}_{1} \in \bar{V}_{\mho_{1}}, v_{2} \in \bar{V}_{\mho_{2}}\right\}$ on $X_{1} \times X_{2}$ by $\bar{V}_{\mho_{1}} \otimes \bar{V}_{\mho_{2}}$.

(a) $\left(\mathscr{V}_{1}\right)_{0} C\left(X_{1}\right) \otimes_{\varepsilon}\left(\mathscr{V}_{2}\right)_{0} C\left(X_{2}\right)$ is a dense topological linear subspace of $C\left(\bar{V}_{\mho_{1}} \otimes \bar{V}_{\mho_{2}}\right)_{0}\left(X_{1} \times X_{2}\right)$.

(b) $\left(\mathscr{V}_{1}\right)_{0} C\left(X_{1}\right) \ddot{\otimes}_{\varepsilon}\left(\mho_{2}\right)_{0} C\left(X_{2}\right)=C\left(\bar{V}_{\mho_{1}} \otimes \bar{V}_{\mho_{2}}\right)_{0}\left(X_{1} \times X_{2}\right)$ if, and only if, $C\left(\bar{V}_{\mho_{1}} \otimes \bar{V}_{\mho_{2}}\right)_{0}\left(X_{1} \times X_{2}\right)$ is complete.

(c) Whenever $\mathcal{K}\left(X_{i}\right) \leqslant V_{n, i}, n=1,2, \ldots, i=1,2$,

$$
\begin{aligned}
\left(\mho_{1}\right)_{0} C\left(X_{1}\right) \check{\otimes}_{\varepsilon}\left(\widetilde{V}_{2}\right)_{0} C\left(X_{2}\right) & =C\left(\bar{V}_{\mho_{1}}\right)_{0}\left(X_{1}\right) \check{\otimes}_{\varepsilon} C\left(\bar{V}_{\mho_{2}}\right)_{0}\left(X_{2}\right) \\
& =C\left(\bar{V}_{\mho_{1}}\right)_{0}\left(X_{1}\right) \varepsilon C\left(\bar{V}_{\mho_{2}}\right)_{0}\left(X_{2}\right) \\
& =C\left(\bar{V}_{\mho_{1}} \otimes \bar{V}_{\mho_{2}}\right)_{0}\left(X_{1} \times X_{2}\right)
\end{aligned}
$$

Proof. (a) By 1.3(a), $\left(\mathscr{V}_{i}\right)_{0} C\left(X_{i}\right)$ is a dense topological linear subspace of $C\left(\bar{V}_{\mathscr{V}_{i}}\right)_{0}\left(X_{i}\right), i=1,2$, and hence $\left(\mathscr{V}_{1}\right)_{0} C\left(X_{1}\right) \otimes_{\varepsilon}\left(\mathscr{V}_{2}\right)_{0} C\left(X_{2}\right)$ forms a dense topological subspace of $C\left(\bar{V}_{V_{1}}\right)_{0}\left(X_{1}\right) \otimes_{\varepsilon} C\left(\bar{V}_{\mho_{2}}\right)_{0}\left(X_{2}\right)$ which, in turn, is a dense topological subspace of $C\left(\bar{V}_{\mho_{1}} \otimes \bar{V}_{\mho_{2}}\right)_{0}\left(X_{1} \times X_{2}\right)$ (e.g., see $\left.[4,1.1]\right)$.

(b) and (c) are immediate from (a) because $\mathcal{K}\left(X_{1}\right) \leqslant V_{n, 1}$ and $\mathcal{K}\left(X_{2}\right) \leqslant V_{n, 2}$, $n=1,2, \ldots$, imply $\mathcal{K}\left(X_{1}\right) \leqslant \bar{V}_{\mho_{1}}$ and $\mathcal{K}\left(X_{2}\right) \leqslant \bar{V}_{\mho_{2}}$, whereby $\mathscr{K}\left(X_{1} \times X_{2}\right) \leqslant \bar{V}_{\mho_{1}} \otimes$ $\bar{V}_{\mho_{2}}$.

For $X_{i}$ and $\mathfrak{V}_{i}=\left\{V_{n, i}\right\}_{n}, i=1,2$, as in Proposition 3.4, we will denote the system $\left\{v_{n, 1} \otimes v_{n, 2} ; v_{n, 1} \in V_{n, 1}, v_{n, 2} \in V_{n, 2}\right\}$ of weights on $X_{1} \times X_{2}$ by $V_{n, 1} \otimes V_{n, 2}$; $V_{n+1,1} \otimes V_{n+1,2} \leqslant V_{n, 1} \otimes V_{n, 2}, n=1,2, \ldots$. Letting $\mathfrak{V}_{1} \otimes \mathscr{V}_{2}$ denote the sequence $\left\{V_{n, 1} \otimes V_{n, 2}\right\}_{n \in \mathrm{N}}$, we may form the inductive limit space $\left(\mathscr{V}_{1} \otimes \mathscr{V}_{2}\right)_{0} C\left(X_{1} \times X_{2}\right)$, and we would now begin to study the relation of the $\varepsilon$-tensor product $\left(\mathscr{V}_{1}\right)_{0} C\left(X_{1}\right) \check{\otimes}_{\varepsilon}\left(\mathscr{V}_{2}\right)_{0} C\left(X_{2}\right)$ with this space.

3.5. LEMMA. (a) The containment $\bar{V}_{\mho_{1}} \otimes \bar{V}_{\mho_{2}} \subset \bar{V}_{\mho_{1} \otimes V_{2}}$ always holds, and hence

$$
\left(\widetilde{V}_{1} \otimes \mho_{2}\right)_{0} C\left(X_{1} \times X_{2}\right) \subset C\left(\bar{V}_{\mho_{1} \otimes V_{2}}\right)_{0}\left(X_{1} \times X_{2}\right) \subset C\left(\bar{V}_{\mho_{1}} \otimes \bar{V}_{\mho_{2}}\right)_{0}\left(X_{1} \times X_{2}\right)
$$

with the inclusion mappings being continuous.

(b) Let $\mathfrak{V}_{i}=\left\{v_{n, i}\right\}_{n \in \mathrm{N}}$ denote decreasing sequences of weights $v_{n, i}$ on $X_{i}$ for $i=1,2$, and let $\mathfrak{V}_{1} \otimes \mathscr{V}_{2}=\left\{v_{n, 1} \otimes v_{n, 2}\right\}_{n \in \mathbf{N}}$. Then $\bar{V}_{\mathscr{V}_{1} \otimes \mathscr{V}_{2}} \leqslant \bar{V}_{\mathscr{V}_{1}} \otimes \bar{V}_{\mathscr{V}_{2}}$ whence

$$
C\left(\bar{V}_{\mho_{1} \otimes V_{2}}\right)_{0}\left(X_{1} \times X_{2}\right)=C\left(\bar{V}_{\mho_{1}} \otimes \bar{V}_{\mho_{2}}\right)_{0}\left(X_{1} \times X_{2}\right)
$$

algebraically and topologically.

Proof. (a) Choose $v_{1}=\inf \left\{\alpha_{n} v_{n, 1} ; n \in \mathbf{N}\right\} \in \check{V}_{\mathscr{V}_{1}}, v_{2}=\inf \left\{\beta_{m} v_{m, 2} ; m \in \mathbf{N}\right\} \in$ $\check{V}_{\mho_{2}}$, where $\alpha_{n}, \beta_{m}>0, v_{n, 1} \in V_{n, 1}, v_{m, 2} \in V_{m, 2}, n, m=1,2, \ldots$. Then obviously $v_{1} \otimes v_{2} \leqslant \inf \left\{\left(\alpha_{n} \beta_{n}\right)\left(v_{n, 1} \otimes v_{n, 2}\right) ; n \in \mathbf{N}\right\} \in \bar{V}_{\mho_{1} \otimes V_{2}}$ whereby $v_{1} \otimes v_{2} \in \bar{V}_{\mho_{1} \otimes V_{2}}$. But this implies $\bar{V}_{\mho_{1}} \otimes \bar{V}_{\mho_{2}} \subset \bar{V}_{\mho_{1} \otimes \Upsilon_{2}}$. 
(b) Fix $v=\inf \left\{\gamma_{n} v_{n, 1} \otimes v_{n, 2} ; n \in \mathbf{N}\right\} \in \check{V}_{\mho_{1} \otimes \mathscr{V}_{2}}$. Then, choosing $\alpha_{n}, \beta_{n} \geqslant$ $\max \left(\gamma_{n}, 1\right), n=1,2, \ldots$, for any $n, m \in \mathbf{N}$, we obtain

$$
\frac{v}{v_{n, 1} \otimes v_{m, 2}} \leqslant \frac{v}{v_{\max (n, m), 1} \otimes v_{\max (n, m), 2}} \leqslant \gamma_{\max (n, m)} \leqslant \alpha_{n} \beta_{m}
$$

whence $v \leqslant \inf \left\{\alpha_{n} v_{n, 1} ; n \in \mathbf{N}\right\} \otimes \inf \left\{\beta_{m} v_{m, 2} ; \quad m \in \mathbf{N}\right\} \in \check{V}_{\mho_{1}} \otimes \check{V}_{\mho_{2}} \subset \bar{V}_{\mho_{1}} \otimes \bar{V}_{\mho_{2}} ;$ the fact that $\bar{V}_{\mho_{1} \otimes \mho_{2}} \leqslant \bar{V}_{\mho_{1}} \otimes \bar{V}_{\mho_{2}}$ now clearly follows.

In the setting of Lemma $3.5(\mathrm{~b}), C\left(\bar{V}_{\mho_{1}} \otimes \bar{V}_{\mho_{2}}\right)_{0}\left(X_{1} \times X_{2}\right)$ can be replaced by $\left(\mathscr{V}_{1} \otimes \mathscr{V}_{2}\right)_{0} C\left(X_{1} \times X_{2}\right)$ in the statements of Theorem 3.4(a) and (b), as can be seen by combining 3.4(a), 3.5(b) and 1.3(a).

On the other hand, the following example shows that Lemma 3.5(b) can fail to hold for sequences $\mathfrak{V}=\left\{V_{n}\right\}_{n}$ of systems of weights.

EXAMPLE. Consider

$$
\begin{aligned}
E=\left\{e=\left(e_{n}\right)_{n} \in c_{0}=C_{0}(\mathbf{N}) ;\right. \\
\quad e \text { is a monotonically decreasing sequence of positive numbers }\},
\end{aligned}
$$

and put $X_{1}=X_{2}=E$ with the discrete topology. For each $n \in \mathbf{N}$, set $v_{n, 1}(e)=e_{n}$ for all $e=\left(e_{n}\right) \in X_{1}$, and note that $\mathcal{V}_{1}=\left\{v_{n, 1}\right\}_{n \in \mathbf{N}}$ is a decreasing sequence of (continuous) weights on $X_{1}$. Further, put $V_{n, 2}=\left\{\lambda / v_{j, 1} ; \lambda>0, j \in \mathbf{N}\right\}, n=1,2, \ldots$, and observe that $\mathscr{V}_{2}=\left\{V_{n, 2}\right\}_{n \in \mathbf{N}}$ is also a decreasing sequence of systems of (continuous) weights on $X_{2}$.

Now, taking $v=\inf \left\{v_{n, 1} \otimes 1 / v_{n, 1} ; n \in \mathbf{N}\right\} \in \check{V}_{\mho_{1} \otimes \mathscr{V}_{2}} \subset \bar{V}_{\mathscr{V}_{1} \otimes \mathscr{V}_{2}}$, let us suppose that there exist $\tilde{u}_{1} \in \bar{V}_{\mho_{1}}$ and $\tilde{u}_{2} \in \bar{V}_{\mho_{2}}$ such that $v \leqslant \tilde{u}_{1} \otimes \tilde{u}_{2}$. Then there are sequences $\left\{\alpha_{n}\right\}_{n \in \mathbf{N}}$ and $\left\{\beta_{n}\right\}_{n \in \mathbf{N}}$ of positive numbers (without loss of generality, we can assume $\left(\alpha_{n}\right)_{n}$ is monotonically increasing with $\left.\lim _{n \rightarrow \infty} \alpha_{n}=+\infty\right)$ and $u_{n, 2} \in V_{n, 2}$, $n \in \mathbf{N}$, so that $v \leqslant u_{1} \otimes u_{2}$ where $u_{1}=\inf \left\{\alpha_{n} v_{n, 1} ; n \in \mathbf{N}\right\}$ and $u_{2}=\inf \left\{\beta_{n} u_{n, 2}\right.$; $n \in \mathbf{N}\}$. Since $v(e, e)=1$ for all $e \in E$, it follows that $u_{1}(e)>0$ for each $e \in E$. However, putting $f_{n}=1 / \alpha_{n}^{2}, n=1,2, \ldots$, we have that $f=\left(f_{n}\right)_{n} \in E$ while $u_{1}(f)=$ $\inf \left\{\alpha_{n} v_{n, 1}(f) ; n \in \mathbf{N}\right\}=\inf \left\{1 / \alpha_{n} ; n \in \mathbf{N}\right\}=0$ which is the desired contradiction.

Let us remark at this point that, in the preceding example, $C\left(\bar{V}_{\mho_{1} \otimes \mho_{2}}\right)_{0}\left(X_{1} \times X_{2}\right)$ $\subset C\left(\bar{V}_{\mho_{1}} \otimes \bar{V}_{\mho_{2}}\right)_{0}\left(X_{1} \times X_{2}\right)$ with continuous inclusion, but that we cannot have $C\left(\bar{V}_{\mho_{1} \otimes \mho_{2}}\right)_{0}\left(X_{1} \times X_{2}\right)=C\left(\bar{V}_{\mathscr{V}_{1}} \otimes \bar{V}_{\mathscr{V}_{2}}\right)_{0}\left(X_{1} \times X_{2}\right)$ topologically by [34, Theorem 3.3].

3.6. Lemma. Let $\mathfrak{V}_{i}=\left\{v_{n, i}\right\}_{n \in \mathbf{N}}$ denote decreasing sequences of weights $v_{n, i}$ on $X_{i}$, $i=1,2$, such that $\inf \left\{v_{n, i}(x) ; x \in K_{i}\right\}>0$ for each compact subset $K_{i}$ of $X_{i}, n=$ $1,2, \ldots$, and $i=1,2$. Then we have the following:

(a) $\mathcal{V}_{1} \otimes \mathcal{V}_{2}$ is regularly decreasing if, and only if, both $\mathcal{V}_{1}$ and $\mathcal{V}_{2}$ are regularly decreasing;

(b) $\mathfrak{V}_{1} \otimes \mathscr{V}_{2}$ satisfies condition (V) if, and only if, both $\mathcal{V}_{1}$ and $\mathfrak{V}_{2}$ do also.

Proof. (a) Let us assume that, given $n \in \mathbf{N}$, there exists $m \geqslant n$ such that, for every $\varepsilon>0$ and every $k \geqslant m$, it is possible to find $\delta(k, \varepsilon)>0$ with

$$
\frac{v_{m, 1}\left(x_{1}\right) v_{m, 2}\left(x_{2}\right)}{v_{n, 1}\left(x_{1}\right) v_{n, 2}\left(x_{2}\right)} \geqslant \varepsilon \Rightarrow \frac{v_{k, 1}\left(x_{1}\right) v_{k, 2}\left(x_{2}\right)}{v_{n, 1}\left(x_{1}\right) v_{n, 2}\left(x_{2}\right)} \geqslant \delta(k, \varepsilon) .
$$


Fixing $n \in \mathbf{N}, m \geqslant n$ as above, $\varepsilon>0, k \geqslant m$, and $x_{2} \in X_{2}$, we put

$$
\delta_{1}(k, \varepsilon)=\delta\left(k, \varepsilon \frac{v_{m, 2}\left(x_{2}\right)}{v_{n, 2}\left(x_{2}\right)}\right) \frac{v_{n, 2}\left(x_{2}\right)}{v_{k, 2}\left(x_{2}\right)} \text {. }
$$

Then $v_{m, 1}\left(x_{1}\right) \geqslant \varepsilon v_{n, 1}\left(x_{1}\right)$ implies

$$
v_{m, 1}\left(x_{1}\right) v_{m, 2}\left(x_{2}\right) \geqslant\left(\varepsilon \frac{v_{m, 2}\left(x_{2}\right)}{v_{n, 2}\left(x_{2}\right)}\right) v_{n, 1}\left(x_{1}\right) v_{n, 2}\left(x_{2}\right),
$$

and hence

$$
v_{k, 1}\left(x_{1}\right) v_{k, 2}\left(x_{2}\right) \geqslant \delta\left(k, \varepsilon \frac{v_{m, 2}\left(x_{2}\right)}{v_{n, 2}\left(x_{2}\right)}\right) v_{n, 1}\left(x_{1}\right) v_{n, 2}\left(x_{2}\right)
$$

from which we obtain $v_{k, 1}\left(x_{1}\right) \geqslant \delta_{1}(k, \varepsilon) v_{n, 1}\left(x_{1}\right)$. Thus $\mathcal{V}_{1}$ is regularly decreasing, and we can conclude in exactly the same way that $\mathcal{V}_{2}$ is regularly decreasing.

In the other direction, let $\mathcal{V}_{1}$ and $\mathcal{V}_{2}$ be regularly decreasing; i.e., given $n \in \mathbf{N}$ there exist $m_{1}, m_{2} \geqslant n$ such that for every $\varepsilon>0$ and every $k \geqslant m_{i}$, it is possible to find $\delta_{i}(k, \varepsilon)>0$ with $v_{m_{i}, i}\left(x_{i}\right) \geqslant \varepsilon v_{n, i}\left(x_{i}\right)$ implying $v_{k, i}\left(x_{i}\right) \geqslant \delta_{i}(k, \varepsilon) v_{n, i}\left(x_{i}\right), i=$ 1,2. Putting $m=\max \left(m_{1}, m_{2}\right)$ and $\delta(k, \varepsilon)=\delta_{1}(k, \varepsilon) \delta_{2}(k, \varepsilon), k \geqslant m$, we observe that $v_{n, i} \geqslant v_{m_{i}, i} \geqslant v_{m, i}$. Hence $v_{m, 1}\left(x_{1}\right) v_{m, 2}\left(x_{2}\right) \geqslant \varepsilon v_{n, 1}\left(x_{1}\right) v_{n, 2}\left(x_{2}\right)$ for some $\varepsilon>0$ yields $v_{m_{1}, 1}\left(x_{1}\right) \geqslant \varepsilon v_{n, 1}\left(x_{1}\right)$ and $v_{m_{2}, 2}\left(x_{2}\right) \geqslant \varepsilon v_{n, 2}\left(x_{2}\right)$ whereby, for every $k \geqslant m$, both $v_{k, 1}\left(x_{1}\right) \geqslant \delta_{1}(k, \varepsilon) v_{n, 1}\left(x_{1}\right)$ and $v_{k, 2}\left(x_{2}\right) \geqslant \delta_{2}(k, \varepsilon) v_{n, 2}\left(x_{2}\right)$. The two inequalities yield $v_{k, 1}\left(x_{1}\right) v_{k, 2}\left(x_{2}\right) \geqslant \delta(k, \varepsilon) v_{n, 1}\left(x_{1}\right) v_{n, 2}\left(x_{2}\right)$ whence $\mathscr{V}_{1} \otimes \mathscr{V}_{2}$ is regularly decreasing.

(b) The argument is similar, and just as easy, as the one preceding. (Also, see the remark after 4.10 in [7].)

After these preparations, we proceed to the next result.

3.7. TheOREM. Let $X_{i}$ denote a locally compact Hausdorff space and take $\mathfrak{V}_{i}=$ $\left\{v_{n, i}\right\}_{n \in \mathrm{N}}$ to be a decreasing sequence of weights $v_{n, i}$ on $X_{i}$ such that $\inf \left\{v_{n, i}(x)\right.$; $\left.x \in K_{i}\right\}>0$ for each compact subset $K_{i}$ of $X_{i}, n=1,2, \ldots, i=1,2$. If both $\mathfrak{V}_{1}$ and $\mathfrak{V}_{2}$ are regularly decreasing, we obtain

$$
\begin{aligned}
\left(\mathscr{V}_{1}\right)_{0} C\left(X_{1}\right) \varepsilon\left(\mathscr{V}_{2}\right)_{0} C\left(X_{2}\right) & =\left(\mathscr{V}_{1}\right)_{0} C\left(X_{1}\right) \check{\otimes}_{\varepsilon}\left(\mathscr{V}_{2}\right)_{0} C\left(X_{2}\right) \\
& =\left(\mathscr{V}_{1} \otimes \mathfrak{V}_{2}\right)_{0} C\left(X_{1} \times X_{2}\right) .
\end{aligned}
$$

Conversely, if the weights $v_{n, i}, n=1,2, \ldots, i=1,2$, are all continuous, then the second of these topological vector space isomorphisms implies that both $\mathcal{V}_{1}$ and $\mathcal{V}_{2}$ are regularly decreasing.

Proof. By Theorem 3.4(c) and Lemma 3.5(b), the second equality holds if, and only if

$$
\left(\mathscr{V}_{1} \otimes \mathscr{V}_{2}\right)_{0} C\left(X_{1} \times X_{2}\right)=C\left(\bar{V}_{\mho_{1} \otimes \mho_{2}}\right)_{0}\left(X_{1} \times X_{2}\right)
$$

But from Theorem 2.3, this is satisfied whenever $\mathcal{V}_{1} \otimes \mathcal{V}_{2}$ is regularly decreasing, while Theorem 2.5 asserts that, conversely,

$$
\left(\mho_{1} \otimes \mathscr{V}_{2}\right)_{0} C\left(X_{1} \times X_{2}\right)=C\left(\bar{V}_{\mho_{1} \otimes \mho_{2}}\right)_{0}\left(X_{1} \times X_{2}\right)
$$


implies $\mathfrak{V}_{1} \otimes \mathscr{V}_{2}$ is regularly decreasing whenever all the weights $v_{n, i}(i=1,2$, $n=1,2, \ldots)$ are continuous. It now only remains to apply Lemma 3.6(a).

A different proof of the following corollary was given in $[7,4.9]$.

3.8. Corollary. Let $X_{i}$ and $\mathfrak{V}_{i}, i=1,2$, be as in Theorem 3.7, and let $\left(A_{i}\left(X_{i}\right)\right.$, co) denote a semi-Montel subspace of $\left(C\left(X_{i}\right), c 0\right)$ for $i=1,2$. If we assume that both $\mathfrak{V}_{1}$ and $\mho_{2}$ satisfy condition $(\mathrm{V})$ and put

$$
\begin{array}{r}
A_{1,2}\left(X_{1} \times X_{2}\right)=\left\{f \in C\left(X_{1} \times X_{2}\right) ; f\left(\cdot, x_{2}\right) \in A_{1}\left(X_{1}\right) \text { and } f\left(x_{1}, \cdot\right) \in A_{2}\left(X_{2}\right)\right. \\
\text { for every pair } \left.\left(x_{1}, x_{2}\right) \in X_{1} \times X_{2}\right\},
\end{array}
$$

then the following topological isomorphism holds:

$$
\left(\mathscr{V}_{1}\right)_{0} A_{1}\left(X_{1}\right) \varepsilon\left(\mathscr{V}_{2}\right)_{0} A_{2}\left(X_{2}\right)=\left(\mathscr{V}_{1} \otimes \mathscr{V}_{2}\right)_{0} A_{1,2}\left(X_{1} \times X_{2}\right)
$$

Proof. From Theorem 1.6, we have

$$
\left(\mathscr{V}_{1}\right)_{0} A_{1}\left(X_{1}\right)=A_{1}\left(\bar{V}_{\mho_{1}}\right)_{0}\left(X_{1}\right) \text { and }\left(\mathscr{V}_{2}\right)_{0} A_{2}\left(X_{2}\right)=A_{2}\left(\bar{V}_{\mho_{2}}\right)_{0}\left(X_{2}\right)
$$

whence

$$
\begin{aligned}
\left(\mathscr{V}_{1}\right)_{0} A_{1}\left(X_{1}\right) \varepsilon\left(\mathscr{V}_{2}\right)_{0} A_{2}\left(X_{2}\right) & =A_{1}\left(\bar{V}_{\mho_{1}}\right)_{0}\left(X_{1}\right) \varepsilon A_{2}\left(\bar{V}_{\mho_{2}}\right)_{0}\left(X_{2}\right) \\
& =A_{1,2}\left(\bar{V}_{\mho_{1}} \otimes \bar{V}_{\mho_{2}}\right)_{0}\left(X_{1} \times X_{2}\right)
\end{aligned}
$$

follows from the slice product theorem [4, 3.2]. Applying Lemma 3.5(b), we see that $\left(\mathscr{V}_{1}\right)_{0} A_{1}\left(X_{1}\right) \varepsilon\left(\mathscr{V}_{2}\right)_{0} A_{2}\left(X_{2}\right)=A_{1,2}\left(\bar{V}_{\mho_{1} \otimes \mho_{2}}\right)_{0}\left(X_{1} \times X_{2}\right)$. On the other hand, $\mathscr{V}_{1} \otimes \mathscr{V}_{2}$ also satisfies condition (V) by Lemma 3.6(b), while it is not hard to prove that $\left(A_{1,2}\left(X_{1} \times X_{2}\right)\right.$, co $)$ is a semi-Montel subspace of $\left(C\left(X_{1} \times X_{2}\right)\right.$, co); e.g., by the slice product theorem, $\left(A_{1,2}\left(X_{1} \times X_{2}\right)\right.$, co $)$ is nothing but $\left(A_{1}\left(X_{1}\right), \mathrm{co}\right) \varepsilon\left(A_{2}\left(X_{2}\right), \mathrm{co}\right)$, and it is known from [23, II, §44, 3.] that the $\varepsilon$-product of semi-Montel spaces is again semi-Montel. Instead of making use of such an abstract permanence property of the $\varepsilon$-product, however, it is somewhat easier in our case to directly verify that $\left(A_{1,2}\left(X_{1} \times X_{2}\right)\right.$, co) is a semi-Montel space, using, say, the Arzelà-Ascoli theorem along with the local compactness of $X_{1}$ and $X_{2}$. We may now apply Theorem 1.6 once more to conclude that $A_{1,2}\left(\bar{V}_{\mho_{1} \otimes \mho_{2}}\right)_{0}\left(X_{1} \times X_{2}\right)=\left(\mathscr{V}_{1} \otimes \mathscr{V}_{2}\right)_{0} A_{1,2}\left(X_{1} \times X_{2}\right)$, which serves to prove our assertion.

Let us remark at this point that if $A_{1}\left(X_{1}\right)$ and $A_{2}\left(X_{2}\right)$ denote the spaces of holomorphic functions on open subsets $X_{1}$ and $X_{2}$ of $\mathbf{C}^{N_{1}}$ and $\mathbf{C}^{N_{2}}$, respectively, then $A_{1,2}\left(X_{1} \times X_{2}\right)$ is exactly the space of holomorphic functions on $X_{1} \times X_{2}$. For more examples and information on other "slice properties", we refer to [5, II, §III].

3.9. Corollary. Let $X_{i}$ and $\mathfrak{V}_{i}, i=1,2$, be as in Theorem 3.7, let $\left(A\left(X_{2}\right)\right.$, co) denote a semi-Montel subspace of $\left(C\left(X_{2}\right), \mathrm{co}\right)$, and define

$$
C A\left(X_{1} \times X_{2}\right)=\left\{f \in C\left(X_{1} \times X_{2}\right) ; f\left(x_{1}, \cdot\right) \in A\left(X_{2}\right) \text { for each } x_{1} \in X_{1}\right\} .
$$

We also assume that $\mathcal{V}_{2}$ satisfies condition $(\mathrm{V})$.

(a) Then $\left(\mathscr{V}_{1}\right)_{0} C\left(X_{1}\right) \check{\otimes}_{\varepsilon}\left(\mathscr{V}_{2}\right)_{0} A\left(X_{2}\right)=C A\left(\bar{V}_{\mho_{1} \otimes V_{2}}\right)_{0}\left(X_{1} \times X_{2}\right)$

(b) If $\mathscr{V}_{1}$ is regularly decreasing, either space in (a) is topologically isomorphic to $\left(\mathscr{V}_{1}\right)_{0} C\left(X_{1}\right) \varepsilon\left(\mathscr{V}_{2}\right)_{0} A\left(X_{2}\right)$, which in turn equals $\left(\mathscr{V}_{1} \otimes \mathscr{V}_{2}\right)_{0} C A\left(X_{1} \times X_{2}\right)=$ ind $_{n \rightarrow} C A\left(v_{n, 1} \otimes v_{n, 2}\right)_{0}\left(X_{1} \times X_{2}\right)$ algebraically and has the topology induced by $\left(\mathcal{V}_{1} \otimes \mathcal{V}_{2}\right)_{0} C\left(X_{1} \times X_{2}\right)$ 
Proof. Part (a) follows from Theorems 1.3(a), 1.6, the slice product theorem, and Lemma 3.5(b) in the "usual" way; i.e.,

$$
\begin{aligned}
& \left(\mho_{1}\right)_{0} C\left(X_{1}\right) \check{\otimes}_{\varepsilon}\left(\mho_{2}\right)_{0} A\left(X_{2}\right)=C\left(\bar{V}_{\mho_{1}}\right)_{0}\left(X_{1}\right) \check{\otimes}_{\varepsilon} A\left(\bar{V}_{\Upsilon_{2}}\right)_{0}\left(X_{2}\right) \\
& =C A\left(\bar{V}_{\mho_{1}} \otimes \bar{V}_{\mho_{2}}\right)_{0}\left(X_{1} \times X_{2}\right)=C A\left(\bar{V}_{\mho_{1} \otimes \mho_{2}}\right)_{0}\left(X_{1} \times X_{2}\right) .
\end{aligned}
$$

For (b), if $\mathscr{V}_{1}$ is regularly decreasing, we have $\left(\mathscr{V}_{1}\right)_{0} C\left(X_{1}\right)=C\left(\bar{V}_{\mho_{1}}\right)_{0}\left(X_{1}\right)$ by Theorem 2.3; since this space is complete and has the a.p., we have that the $\varepsilon$-product and the $\varepsilon$-tensor product agree. The algebraic identity

$$
\left(\mathscr{V}_{1}\right)_{0} C\left(X_{1}\right) \varepsilon\left(\mathscr{V}_{2}\right)_{0} A\left(X_{2}\right)=\left(\mathscr{V}_{1} \otimes \mathscr{V}_{2}\right)_{0} C A\left(X_{1} \times X_{2}\right)
$$

follows from [7, 4.6], noting that $\left(\mathscr{V}_{2}\right)_{0} A\left(X_{2}\right)$ is a Silva space because of condition (V). Finally, $\left(\mathscr{V}_{2}\right)_{0} A\left(X_{2}\right)$ is a topological linear subspace of $\left(\mathscr{V}_{2}\right)_{0} C\left(X_{2}\right)$ in view of $0.4(\mathrm{~d})$, and hence $\left(\mathscr{V}_{1}\right)_{0} C\left(X_{1}\right) \varepsilon\left(\mathscr{V}_{2}\right)_{0} A\left(X_{2}\right)$ is a topological subspace of $\left(\mathscr{V}_{1}\right)_{0} C\left(X_{1}\right) \varepsilon\left(\mathscr{V}_{2}\right)_{0} C\left(X_{2}\right)=\left(\mathscr{V}_{1} \otimes \mathscr{V}_{2}\right)_{0} C\left(X_{1} \times X_{2}\right)($ see 3.7)

In [7, 4.10], it was remarked that, under the conditions of 3.9(b),

$$
\left(\mathscr{V}_{1}\right)_{0} C\left(X_{1}\right) \check{\otimes}_{\varepsilon}\left(\mathscr{V}_{2}\right)_{0} A\left(X_{2}\right)=\left(\mathscr{V}_{1} \otimes \mathscr{V}_{2}\right)_{0} C A\left(X_{1} \times X_{2}\right)
$$

holds algebraically and topologically whenever $\left(\mathfrak{V}_{2}\right)_{0} A(X)$ is a nuclear space. Similarly, in the setting of 3.8,

$$
\left(\mathscr{V}_{1}\right)_{0} A_{1}\left(X_{1}\right) \varepsilon\left(\mathscr{V}_{2}\right)_{0} A\left(X_{2}\right)=\left(\mathscr{V}_{1} \otimes \mathscr{V}_{2}\right)_{0} A_{1,2}\left(X_{1} \times X_{2}\right)
$$

holds whenever $\left(\mathfrak{V}_{1}\right)_{0} A_{1}\left(X_{1}\right)$ and $\left(\mathfrak{V}_{2}\right)_{0} A_{2}\left(X_{2}\right)$ are complete spaces with one of them being nuclear. (In this case, it is not necessary to assume that both $\mathcal{V}_{1}$ and $\mathcal{V}_{2}$ satisfy $(\mathrm{V})$ nor that both $\left(A_{1}\left(X_{1}\right), \mathrm{co}\right)$ and $\left(A_{2}\left(X_{2}\right), \mathrm{co}\right)$ are semi-Montel.)

Using induction, there is an additional remark which can be made as an immediate consequence of Theorem 3.7 (and well-known properties of the $\varepsilon$-product): Let $X_{i}$ denote locally compact Hausdorff spaces, and take $\mathscr{V}_{i}=\left\{v_{n, i}\right\}_{n \in \mathbf{N}}$ to be decreasing sequences of weights $v_{n, i}$ on $X_{i}$ such that $\inf \left\{v_{n, i}(x) ; x \in K_{i}\right\}>0$ for each compact subset $K_{i}$ of $X_{i}, n=1,2, \ldots, i=1, \ldots, N$. If all the sequences $\mathscr{V}_{i}$ are regularly decreasing, then the following topological isomorphisms hold:

$$
\begin{gathered}
\left(\mathscr{V}_{1}\right)_{0} C\left(X_{1}\right) \varepsilon \cdots \varepsilon\left(\mathscr{V}_{N}\right)_{0} C\left(X_{N}\right)=\left(\mathscr{V}_{1}\right)_{0} C\left(X_{1}\right) \check{\otimes}_{\varepsilon} \cdots \check{\otimes}_{\varepsilon}\left(\mathscr{V}_{N}\right)_{0} C\left(X_{N}\right) \\
=\left(\mathscr{V}_{1} \otimes \cdots \otimes \mathcal{V}_{N}\right)_{0} C\left(X_{1} \times \cdots \times X_{N}\right) .
\end{gathered}
$$

To conclude this section, let us point out that, in [24], one of the authors studied nuclear inductive limits of weighted spaces of holomorphic functions and topological tensor products of such limits with regard to applications in the theory of representation of distributions in several variables as boundary values of holomorphic functions. One aim in [7] was to remove, as far as possible, the nuclearity assumptions in the topological tensor product results of [24] by considering $\varepsilon$-products instead of $\pi$-tensor products. The "best" results in this direction were 4.8 and the second part of 4.9 in [7], where only a compactness condition remained; i.e., the decreasing sequence $\mathfrak{V}=\left\{v_{n}\right\}_{n}$ of weights had to satisfy (V). Now, to sum up, our present Theorems 3.1, 3.3, and 3.7 contain much more information, while we are able to recover and improve most of the former tensor product representations for 
spaces of holomorphic functions in 3.2 and 3.8. However, as mentioned above, the latter results are still somewhat incomplete due to the fact (see the Problem in 0.4) that it is not known whether the last assertion of $0.4(\mathrm{~d})$ remains true for, say, holomorphic functions with values in a Banach space.

\section{Examples. Discussion of related work.}

4.1. In the theory of Ehrenpreis [11], a 1.c. space $W$ of functions, or more general objects such as distributions or ultradistributions, on $\mathbf{R}^{N}$ is called analytically uniform $(A U)$ if the following three requirements are satisfied:

(a) There exists a l.c. space $W^{\prime}$ such that $W$ is the dual of $W^{\prime}$ and the topology of $W$ is the strong topology $\beta\left(W, W^{\prime}\right)$.

(b) For each $z \in \mathbf{C}^{N}$, we have $\exp (i\langle\cdot, z\rangle) \in W$, and the map $z \rightarrow \exp (i\langle\cdot, z\rangle)$ is complex analytic from $\mathbf{C}^{N}$ into $W$. Moreover, the span of the exponentials is dense in $W$. Thus, for any $S \in W^{\prime}$, the abstract Fourier transform $\hat{S}$, where

$$
\hat{S}(z)=S(\exp (i\langle\cdot, z\rangle)) \text { for all } z \in \mathbf{C}^{N},
$$

is an entire function; the space $\hat{W}^{\prime}$ of Fourier transforms of elements of $W^{\prime}$ is topologized so as to make the Fourier transform $S \rightarrow \hat{S}$ a topological isomorphism.

(c) There exists a system $V$ of nonnegative continuous functions $v$ on $\mathbf{C}^{N}$, which is termed an $A U$-structure for $W$, such that (in our terminology) $\hat{W}^{\prime}$ is a topological linear subspace of $H V_{0}\left(\mathbf{C}^{N}\right)$.

There may exist many different $A U$-structures for a given $A U$-space. In fact, to prove his main result in Part A of [11], the so-called "Fundamental Principle" (Quotient Structure Theorem, Integral Representation Theorem) for partial differential equations, Ehrenpreis put further conditions on $W$ which imply, for example, that

$$
\hat{W}^{\prime}=H V_{0}\left(\mathrm{C}^{N}\right)=H V\left(\mathrm{C}^{N}\right) \quad \text { algebraically (and topologically), }
$$

and involve being able to choose an $A U$-structure $V$ for $W$ with "localizability" properties which are too technical to be explicitly noted here. An $A U$-space $W$ which satisfies these additional conditions is called product localizable (PLAU).

In their Lecture Notes [2] (which are dedicated to applications of $A U$-spaces to convolution equations), Berenstein and Dostal changed the definition of $A U$-space somewhat. Instead of (c) above, they required that

$$
\hat{W}^{\prime}=H V\left(\mathbf{C}^{N}\right) \text { algebraically and topologically, }
$$

where the functions $v \in V$ are assumed to be strictly positive, as well as adding some other conditions which Ehrenpreis had noted for PLAU-spaces.

An analysis of the existing situation led Berenstein and Dostal [2, p. 5] to the general problem of finding (what they call) "complex representations" for spaces of entire functions. In our terminology, the problem is as follows: Given a l.c. space $\mathscr{F}=\mathscr{F}\left(\mathbf{C}^{N}\right)$ of entire functions on $\mathbf{C}^{N}$ under a topology which is stronger than uniform convergence on compact subsets, when is it possible to find a system $V$ of strictly positive continuous weights $v$ on $\mathbf{C}^{N}$ such that, as topological vector spaces, $\mathscr{F}=H V\left(\mathbf{C}^{N}\right)$ ? If this occurs, $H V\left(\mathbf{C}^{N}\right)$ is termed a complex representation of $\mathscr{F}$, and it is pointed out in [2] that the methods of Ehrenpreis demonstrate the importance of 
concrete complex representations for solving linear equations (or systems of equations) of convolution type in various spaces of functions, distributions and ultradistributions. They further note that it may be important in applications to be able to replace a large system $V$ of weights (such that $H V\left(\mathrm{C}^{N}\right)$ is a complex representation for a given space $\mathscr{F}$ ) by some smaller subsystem which yields the same topological vector space of entire functions and in which the weights all share additional "nice" properties.

In the course of the present development, we have, of course, solved the complex representation problem in one important general case; namely, if $\mathcal{V}=\left\{v_{n}\right\}_{n}$ is a decreasing sequence of strictly positive continuous weights on $\mathbf{C}^{N}$ which satisfies condition $(\mathrm{V})$, then the space $\mathscr{F}=\mathscr{V} H\left(\mathrm{C}^{N}\right)=\mathscr{V}_{0} H\left(\mathrm{C}^{N}\right)$ has the complex representation $H \hat{V}\left(\mathbf{C}^{N}\right)=H \hat{V}_{0}\left(\mathbf{C}^{N}\right)$ as a consequence of $1.6,1.8,1.9$, and 1.11. In fact, some of our work in the latter part of $\S 1$ can be seen as an effort to reduce the maximal system $\bar{V}=\bar{V}_{\mathscr{V}}$ associated with the sequence $\mathfrak{V}=\left\{v_{n}\right\}_{n}$ to the more "manageable" form $\hat{V}=\hat{V}_{\mathscr{V}}$ in which the given "data" $v_{n}$ occur in a more direct way and "nice" properties become easier to derive.

We should also add at this point that our result 1.3(a), (b) on $\mathfrak{V}_{0} C(X)$ clearly seems to open up the possibility of obtaining at least a "weak" complex representation $H \bar{V}_{0}\left(\mathbf{C}^{N}\right)$ for each space $\mho_{0} H\left(\mathbf{C}^{N}\right)$, where $\mathscr{V}=\left\{V_{n}\right\}_{n \in \mathrm{N}}$ is allowed to be a sequence of systems of weights on $\mathbf{C}^{N}$ such that $\mathscr{K} \leqslant V_{n}$ for $n=1,2, \ldots$. However, as we have pointed out before (in the Problem of $\$ 0.4$ ), we do not know whether $\mho_{0} H\left(\mathbf{C}^{N}\right)$ is still a topological subspace of $\Upsilon_{0} C\left(\mathbf{C}^{N}\right)$ in this more general setting.

In both [11] and [2], complex representations have been found for many important nuclear function spaces and (ultra-) distribution spaces by ad hoc methods; it was then proved that the corresponding dual spaces were $A U$ or even PLAU. Let us demonstrate, by way of a special and well-known example, how the question of projective characterizations of weighted inductive limits of entire functions may enter into the proof that a given function space is analytically uniform. Taking $W=\mathcal{E}=\mathcal{E}\left(\mathbf{R}^{N}\right)$ to be the space of all infinitely differentiable functions on $\mathbf{R}^{N}$ under its usual $(F)$-space topology, an appropriate version of the Paley-WienerSchwartz theorem asserts that the Fourier transform is a topological isomorphism of $W^{\prime}=\mathcal{E}^{\prime}$, the space of distributions with compact support endowed with the strong topology $\beta\left(\mathcal{E}^{\prime}, \mathcal{E}\right)$, onto $\hat{W}^{\prime}=\mathcal{V} H\left(\mathbf{C}^{N}\right)$ where $\mathfrak{V}=\left\{v_{n}\right\}_{n \in \mathbf{N}}$ with

$$
v_{n}(z)=\prod_{j=1}^{N} \frac{\exp \left(-n\left|\operatorname{Im}\left(z_{j}\right)\right|\right)}{\left(1+\left|z_{j}\right|\right)^{n}}, \quad z=\left(z_{1}, \ldots, z_{N}\right) \in \mathbf{C}^{N} \text {. }
$$

Since $\mathfrak{V}$ is a decreasing sequence of continuous weights on $\mathbf{C}^{N}$ which satisfies (V), our general results from $\S 1$ apply to yield that $\tilde{V}$ (or $\hat{V}$ ) is an $A U$-structure for $\mathcal{E}$, and so we have a proof of the well-known fact that $\mathcal{E}$ is an $A U$-space in the sense of Ehrenpreis; indeed, $\mathcal{E}$ is $P L A U$ [11]. Similarly, if one tries to prove that certain related spaces of ultradifferentiable functions are $A U$, known Paley-WienerSchwartz-Komatsu theorems reduce the problem to verifying an equality like $\mho_{0} H\left(\mathbf{C}^{N}\right)=H \tilde{V}_{0}\left(\mathbf{C}^{N}\right)$ for a corresponding system $\mathcal{V}=\left\{v_{n}\right\}_{n}$ to which 1.6 and 1.9 again apply. 
The situation for ' $\mathcal{D}^{\prime}$, which is known to be $L A U$ in the terminology of Ehrenpreis (cf. [11]), and the related spaces of ultradistributions, where another Paley-Wiener type theorem may be used to obtain a topological isomorphism between the dual and a weighted inductive limit of entire functions, is more complicated and not yet covered by our results because the above-mentioned topological subspace problem remains open in the $(L F)$-case.

Quite recently, S. Hansen ([18], [19]) has given a new treatment of (the more functional analytic aspects of) the Fundamental Principle of Ehrenpreis in which, roughly speaking, solutions of (systems of) homogeneous linear partial differential equations with constant coefficients have natural integral representations as "superpositions" of exponential-polynomial solutions. As we have already noted, the proof of the Fundamental Principle depends in an essential way on having a complex representation (of the dual) in the sense of Berenstein-Dostal and an $A U$-structure $V$ with certain localizability conditions. Hansen [19], using a new definition of $L A U$-spaces, clarifies the role of these conditions on $V$ and explains how Hörmander's results on cohomology with bounds come into play at this point. In $[19, \S 4]$, also noting that many spaces of entire functions occurring in Fourier analysis $a$ priori arise as weighted inductive limits, Hansen utilizes results and techniques from a preliminary version of the present paper in showing that a large and important class of $(L B)$-spaces of entire functions, or rather their strong duals, can be given $L A U$-structures (in his sense) which define these spaces algebraically and topologically. Combining this with known Paley-Wiener type theorems, he then establishes that $\mathcal{E}(\Omega)$ and the spaces $\mathcal{E}_{\omega}(\Omega)$ and $\mathcal{E}\left(\Omega,\left(M_{p}\right)\right)$ of ultradifferentiable functions (as introduced by Beurling and Roumieu, respectively) are $L A U$-spaces for any convex open set $\Omega$ in $\mathbf{R}^{N}$. We refer the reader to Hansen's interesting and readable articles [18] and [19] which go far beyond the partial results in this direction obtained independently by the present authors.

Let us further note that, also quite recently, O. v. Grudzinski [17] has applied our Theorem 1.6 (together with its refinement 1.9) to conclude that $\mathscr{D}_{3}(\omega, M), \mathscr{V}_{2}(\omega, M)$ and $\mathscr{Y}_{3}(\omega, M)$ (in his terminology), spaces which play an important role in his study of convolution equations, have $A U$-structures.

4.2. The first unifying general result for weighted spaces of entire functions in the direction of our Theorem 1.6, and with applications to analytically uniform spaces, is due to B. A. Taylor [37] (also see [36]); it can be phrased as follows:

Let $\left\{\Phi_{n}\right\}_{n \in \mathrm{N}}$ denote an increasing sequence of plurisubharmonic (p.s.h.) functions on $\mathbf{C}^{N}$, and suppose that

(i) for every $n>1, \Phi_{n+1}-\Phi_{n}$ is bounded above on every bounded set;

(ii) $\Phi_{n+1}(z) \geqslant \log \left(1+\|z\|^{2}\right)+\sup \left\{\Phi_{n}(z+\xi) ;\|\xi\| \leqslant 1\right\}$ on $\mathbf{C}^{N}, n=1,2, \ldots$, where $\|\cdot\|$ denotes the Euclidean norm.

Next, consider the weights $v_{n}=\exp \left(-\Phi_{n}\right), n=1,2, \ldots$, and construct the inductive limit space $\mathscr{V} H\left(\mathbf{C}^{N}\right)=\operatorname{ind}_{n \rightarrow} H v_{n}\left(\mathbf{C}^{N}\right)$ as in 0.1 and 0.2 ; as an associated system of "weights" on $\mathrm{C}^{N}$, Taylor takes

$$
\overline{\bar{V}}=\left\{\overline{\bar{v}} ; 1 / \overline{\bar{v}} \text { is u.s.c. and } 1 / \overline{\bar{v}} \geqslant \varepsilon_{n} \exp \left(\Phi_{n}\right) \text { for some constants } \varepsilon_{n}>0 \text { and all } n\right\} \text {. }
$$


The assertion (Theorem 1 of [37]) is then that the space $H \overline{\bar{V}}\left(C^{N}\right)$, defined as in 0.1, induces the inductive limit topology on $\mathfrak{V} H\left(\mathbf{C}^{N}\right)$. In Taylor's argument, by the way, it is implicit that $\mathscr{V} H\left(\mathbf{C}^{N}\right)=H \bar{V}\left(\mathbf{C}^{N}\right)$ algebraically.

Indeed his proof is interesting from several points of view, and is essentially direct. Roughly speaking, the crux of his argument lies in replacing supremum norms by (weighted) $L_{2}$-norms and the use of Hörmander's existence theorems for solutions of the $\bar{\partial}$-equation with certain growth conditions (from the theory of several complex variables). Subsequently, Servien [32] asserted a more general theorem than Taylor's, claiming to have avoided Hörmander's techniques through a different approach. Unfortunately, Servien's argument has a gap at exactly this point, and it seems unlikely that the result can be obtained along these lines (cf., for example, the reviews of [32]).

For a comparison with our Theorem 1.6 as applied to entire functions, let us remark that, clearly, our setting is different from Taylor's insofar as conditions on "weights" are concerned. Our weights $v_{n}$ are u.s.c., or even continuous, and we impose the same continuity conditions on the elements of the associated systems $\bar{V}$ or $\tilde{V}$, respectively. In contrast, Taylor's weights $v_{n}$ have the special form $\exp \left(-\Phi_{n}\right)$ with $\Phi_{n}$ p.s.h., which is needed in order to apply Hörmander's theory, and he requires the functions $1 / \overline{\bar{v}}$ to be u.s.c., where $\overline{\bar{v}}$ denotes the elements of his system $\overline{\bar{V}}$. Apart from this, however, $\overline{\bar{V}}$ corresponds exactly with our maximal system $\bar{V}$.

Concerning the special form of Taylor's weights, while we do not need plurisubharmonicity in our arguments, whereby this hypothesis is not needed to obtain $A U$-structures in general, it has been pointed out by Hansen [19] that plurisubharmonicity has a significant part in deducing the additional localizability conditions required for the proof of the Fundamental Principle. Since each p.s.h. function is u.s.c. by definition, and hence bounded above on each compact set, Taylor's weights $v_{n}=\exp \left(-\Phi_{n}\right)$ satisfy our assumption

(*) $\inf \left\{v_{n}(x) ; x \in K\right\}>0$ for each compact subset $K$ of $\mathbf{C}^{N}, \quad n=1,2, \ldots$,

while his condition (i) above guarantees that $v_{n+1} / v_{n}=\exp \left(-\left[\Phi_{n+1}-\Phi_{n}\right]\right)$ is bounded away from 0 on each compact set, which is also satisfied in our setting. Next, we remark that condition (ii) obviously implies

$$
\Phi_{n+1}(z) \geqslant \log \left(1+\|z\|^{2}\right)+\Phi_{n}(z)
$$

and hence

$(* *) \quad \frac{v_{n+1}(z)}{v_{n}(z)}=\frac{\exp \left(-\Phi_{n+1}(z)\right)}{\exp \left(-\Phi_{n}(z)\right)} \leqslant \frac{1}{1+\|z\|^{2}} \quad$ on $\mathbf{C}^{N}, \quad n=1,2, \ldots$,

whereby, in particular, the sequence $\mathfrak{V}=\left\{v_{n}\right\}_{n}$ in Taylor's theorem satisfies our condition (V). However, while (V) only yields compactness of the embedding maps $H v_{n}\left(\mathbf{C}^{N}\right) \rightarrow H v_{m}\left(\mathbf{C}^{N}\right)$ when $m=m(n)>n$ is large enough so that it is not possible to infer more than that $\mathfrak{V} H\left(\mathbf{C}^{N}\right)=\widetilde{V}_{0} H\left(\mathbf{C}^{N}\right)$ is a Silva space, the full force of (ii), at least for continuous weights $v_{n}$, implies nuclearity of the embeddings $H v_{n}\left(\mathbf{C}^{N}\right) \rightarrow$ $H v_{k}\left(\mathbf{C}^{N}\right)$, for large enough $k=k(n)>n$, and so, in Taylor's theorem, $\mathfrak{V} H\left(\mathbf{C}^{N}\right)=$ $\mathfrak{V}_{0} H\left(C^{N}\right)$ will be a $(D F N)$-space. (This can be deduced, for example, from [24, §2, 
Satz 2] in a standard way, using (**) and the estimate

$$
\begin{aligned}
v_{n+1}(z) & =\exp \left(-\Phi_{n+1}(z)\right) \leqslant \exp \left(-\sup \left\{\Phi_{n}(z+\xi) ;\|\xi\| \leqslant 1\right\}\right) \\
& =\inf \left\{v_{n}(z+\xi) ;\|\xi\| \leqslant 1\right\},
\end{aligned}
$$

$z \in \mathbf{C}^{N}, n=1,2, \ldots$, which readily follows from (ii).) Note that, in some sense, nuclearity was needed in Taylor's proof so as to replace weighted sup-norms by $L_{2}$-norms, but no such hypothesis is necessary for our arguments. Moreover, condition (V) is much easier to check than condition (ii) above.

As we have already noted, Taylor's argument uses deep results from complex analysis in several variables, while our method is purely functional analytic. Of course, dealing directly with entire functions tends to rule out partition of unity arguments, and it is here, at least from the point of view of applications to weighted inductive limits of holomorphic functions, that we have made a "detour" by first considering the case for continuous functions (Theorem 1.3) where we could make use of the space $C_{c}(X)$ and continuous partitions of unity, and then deducing the result for holomorphic functions (Theorem 1.6) via Baernstein's open mapping lemma [1].

It might be possible, by the way, to extend Taylor's method of proof to include holomorphic functions on (strictly) pseudoconvex domains in $\mathbf{C}^{N}$ (where Hörmander's techniques are still available). But, as one advantage of our method, such restrictions are altogether avoided through an immediate extension to arbitrary open subsets of $\mathbf{C}^{N}$. Part of this paper arose from an effort to find such a generalization of Taylor's result, and Theorem 1.6 accomplishes this task, but we should again mention that many questions still remain with import for the theory of $A U$-spaces, as well as from a more structure theoretic point of view.

4.3. Weighted inductive limits of spaces of holomorphic functions also occur in a very natural way in many applications in complex analysis and spectral theory. Since it is virtually impossible to note all important papers from this area, we shall refer instead to Ferrier's book [12] in which much pertinent material, including some interesting relationships between the work of Waelbroeck, Cnop, and Ferrier in spectral theory along with results in complex analysis due to L. Hörmander, N. Sibony, and B. A. Taylor, among others, can be found.

More specifically, let $\delta$ denote a "weight function" in the terminology of [12]; i.e., a nonnegative continuous function on $\mathbf{C}^{N}$ such that

(W1) $\sup _{z \in \mathbf{C}^{N}}\|z\| \delta(z)<\infty$ and

(W2) $\left|\delta(z)-\delta\left(z^{\prime}\right)\right| \leqslant\left\|z-z^{\prime}\right\|$ for all $z, z^{\prime} \in \mathbf{C}^{N}$,

where $\|\cdot\|$ is the Euclidean norm. Although $\left(\delta^{n}\right)_{n \in N}$ is not necessarily decreasing, since $\delta$ is bounded by a constant $C>0$ on $\mathbf{C}^{N},\left(\delta^{n} / C^{n}\right)_{n \in \mathrm{N}}$ is an "equivalent" decreasing sequence; for the open subset $X=\left\{z \in \mathbf{C}^{N} ; \delta(z)>0\right\}$ let $v_{n}=\delta^{n} /\left.C^{n}\right|_{X}$, $n=1,2, \ldots$, and take $\mathfrak{V}=\left\{v_{n}\right\}_{n \in \mathbf{N}}$. Ferrier [12] uses $\vartheta(\delta)$ to denote our $\mathfrak{V} H(X)$, and a large part of his book is centered around these spaces and their applications in spectral theory and complex analysis; for an instance of concrete examples, see [12, 1.2]. Also note that $\delta$ clearly vanishes at infinity on $X$, and hence $\mathfrak{V}$ satisfies our condition (V). 
Early in his book [12, p. 13], Ferrier says (essentially) that, while it would be possible to consider the inductive limit topology of $\mathcal{V} H(X)$ on $\vartheta(\delta)$, such a topology is not easy to handle, and therefore, roughly speaking, he prefers to work with the system $\left(H v_{n}(X)\right)_{n \in \mathrm{N}}$ of generating spaces itself (rather than going to the limit space). So, instead of standard locally convex theory, the notions of boundedness structures, polynormed vector spaces, and filtrated $b$-spaces are used throughout [12]. But now note that our Theorem 1.6 applies to give a good, explicit description of the inductive limit topology on $\theta(\delta)$ in terms of weighted seminorms.

4.4. There is an interesting connection between Theorem 1.3(d) and a "classical" result due to L. Schwartz. To illustrate this, let us drop the convention that our weights are upper semicontinuous and real valued. Then, taking $X$ to be locally compact and $\sigma$-compact, let $\left(K_{n}\right)_{n \in \mathbf{N}}$ denote a sequence of compact sets such that $X=\cup_{n \in \mathrm{N}} K_{n}$ and $K_{n} \subset \stackrel{\circ}{K}_{n+1}, n=1,2, \ldots$; for each $n \in \mathbf{N}$, put

$$
v_{n}(x)=\left\{\begin{array}{cc}
1, & x \in K_{n} \\
+\infty, & x \notin K_{n}
\end{array}\right\} .
$$

Adopting the usual convention $0(+\infty)=0$, we have that $C\left(v_{n}\right)_{0}(X)=C_{K_{n}}(X)$, the Banach space of all continuous functions on $X$ with support contained in $K_{n}$ under the supremum norm, $n=1,2, \ldots$, while

$$
\mathfrak{V}_{0} C(X)=\operatorname{ind}_{n \rightarrow} C_{K_{n}}(X)=C_{c}(X)
$$

is the space of all continuous functions with compact support under the canonical inductive limit topology. Moreover, $\mathfrak{V}=\left\{v_{n}\right\}_{n}$ satisfies condition (V) in a well-defined way, the system $\tilde{V}=\tilde{V}_{\mathscr{V}}$ (associated with $\mathcal{V}$ exactly as in 0.2 ) consists of all nonnegative continuous functions on $X$, and it is not too hard to show that

$$
C_{c}(X)=C \tilde{V}_{0}(X) \text { algebraically and topologically. }
$$

This result is essentially due to L. Schwartz [30]; a discussion of its possible extensions to more general spaces $X$ and to vector valued functions can also be found in [30, 33, 3, I], and [20, 5.4]. At this point, however, let us note that, for an uncountable discrete space $X, C_{c}(X)$ and $C \tilde{V}_{0}(X)$ are equal algebraically, but have different duals [33].

4.5. We shall conclude this section with two additional remarks. Firstly, throughout the paper, we have concentrated on the inductive limits $\mathcal{V} C(X)$ and $\mathcal{V}_{0} C(X)$, but some interesting consequences for the weighted spaces $C \bar{V}(X)$ and $C \bar{V}_{0}(X)$ have also been obtained; e.g., see 1.3(d), 1.4(c), and 2.3. In fact, if a system $\bar{V}$ of weights on a locally compact space $X$ arises as the maximal system $\bar{V}_{\mathfrak{V}}$ associated with a decreasing sequence $\mathscr{V}=\left\{v_{n}\right\}_{n \in \mathrm{N}}$ of weights $v_{n}$ on $X$ such that $\inf \left\{v_{n}(x) ; x \in K\right\}>0$ for each compact subset $K$ of $X, n=1,2, \ldots$ (that is, if $\bar{V}$ is the set of weights $\bar{v}$ on $X$ such that $\bar{v} / v_{n}$ is bounded for each $n \in \mathbf{N}$ ), then $C \bar{V}_{0}(X)$ is (at least) a barrelled (DF)-space, while if $\mathscr{V}=\left\{v_{n}\right\}_{n}$ is also regularly decreasing, then we know that $C \bar{V}_{0}(X)$ is ultrabornological as well. Furthermore, the remarks preceding 1.12 show, at least when $X$ is $\sigma$-compact, that the space $C \bar{V}(X)$ always has a fundamental sequence of bounded sets (since this is true for $\mathcal{V} C(X)$ and the two spaces have the 
same bounded sets). As for obtaining information about the weighted spaces $C \bar{V}(X)$ and $C \bar{V}_{0}(X)$, a study of uncountable weighted inductive limits (as considered at the very beginning of [7]) could well be worthwhile. Also, we refer to [7, 2.10] for an interesting construction which, in a certain sense, reverses the approach we have taken here and associates an "internal" weighted inductive limit with weighted spaces like $C \bar{V}(X)$; a remark by B. A. Taylor [36] prompted the work in this direction.

Secondly, the sequence spaces $\mathcal{V} C(X)$ and $\mathcal{V}_{0} C(X)$, taking $X=\mathbf{N}$ (or $\mathbf{N} \times \mathbf{N}$ ), which have occurred at various points in the present article (see 1.5, 1.12-13, 2.4(c)) have been studied in other contexts. In fact, the spaces $\mathcal{V} C(\mathbf{N})$ are inductive limits of weighted $l_{\infty}$-spaces, called "co-echelon spaces" in Köthe's terminology, while the inductive limits $\mathcal{V}_{0} C(\mathbf{N})$ of weighted $c_{0}$-spaces have been considered by Köthe, Dubinsky, and many others; in this setting, condition (V) corresponds to the well-known Silva space criterion for co-echelon spaces. Our Theorem 2.6 gives a complete classification of inductive limits of type $\mathfrak{V}_{0} C(X)$ with regard to regularity, bounded retractivity, and completeness in terms of $\mathcal{V}$ being regularly decreasing; even in the case of sequence spaces, these results were apparently not previously known.

Moreover, from our work in $\$ \S 1$ and 2 , it is not too hard to add some new conclusions to the classical theory of echelon and co-echelon spaces. Fixing a decreasing sequence $\mathcal{V}=\left\{v_{n}\right\}_{n}$ of strictly positive weights on $\mathbf{N}$, we let $B=\left(b_{n}\right)_{n}=$ $\left\{1 / v_{n}\right\}_{n \in \mathbf{N}}$ denote the corresponding increasing sequence of sequences and take $\Lambda(B)$ to be the associated echelon space (see the remarks before Theorem 1.13). While it is well-known that $\mathcal{V}_{0} C(\mathbf{N})^{\prime}=\Lambda(B)$ and $\Lambda(B)^{\prime}=\mathscr{V} C(\mathbf{N})(=C \bar{V}(\mathbf{N}))$ algebraically, we can note the following additional facts:

(1) The echelon space $\Lambda(B)$ under its canonical Fréchet topology is the strong dual of $\mathfrak{V}_{0} C(\mathbf{N})$ or $C \bar{V}_{0}(\mathbf{N})$.

(2) The strong dual of $\Lambda(B)$ is equal to the weighted space $C \bar{V}(\mathbf{N})$ (but may differ from $\mathcal{V} C(\mathbf{N})$ topologically).

(3) $\left(\mathfrak{V}_{0} C(\mathbf{N})_{b}^{\prime}\right)_{b}^{\prime}=\left(C \bar{V}_{0}(\mathbf{N})_{b}^{\prime}\right)_{b}^{\prime}=C \bar{V}(\mathbf{N})$ algebraically and topologically.

Proof. (1) Clearly (by polarity), $\beta\left(\Lambda(B), \mathcal{V}_{0} C(\mathbf{N})\right)$ is stronger than the Fréchet topology of $\Lambda(B)$, which therefore coincides with both $\beta(\Lambda(B), \mathcal{V} C(\mathbf{N}))$ and $\beta\left(\Lambda(B), \mathscr{V}_{0} C(\mathbf{N})\right)$. But since $\mathscr{V}_{0} C(\mathbf{N})$ is a $(D F)$-space and $C \bar{V}_{0}(\mathbf{N})=\overline{\mathcal{V}_{0} C(\mathbf{N})}$ by 1.3(b), an observation in [23, I, §29, 5. (3), proof of a)] yields $\mathcal{V}_{0} C(\mathbf{N})_{b}^{\prime}=C \bar{V}_{0}(\mathbf{N})_{b}^{\prime}$.

(2) As the completion of the barrelled space $\mathfrak{V}_{0} C(\mathbf{N}), C \bar{V}_{0}(\mathbf{N})$ is barrelled, and hence a simple polarity argument shows that each bounded subset $A$ of $\Lambda(B)=$ $C \bar{V}_{0}(\mathbf{N})_{b}^{\prime}$ is contained in a bounded set in $\Lambda(B)$ of the form

$$
\left\{x=\left(x_{k}\right)_{k} \in \Lambda(B) ; \sum_{k \in \mathbf{N}} \frac{\left|x_{k}\right|}{\bar{v}(k)} \leqslant 1\right\}, \quad \bar{v} \in \bar{V} .
$$

At this point, another look at the proof that $\Lambda(B)_{b}^{\prime} \rightarrow C \bar{V}(\mathbf{N})$ continuously (see Example 1.12 and the subsequent remarks) reveals that, in fact, $C \bar{V}(\mathbf{N}) \rightarrow \Lambda(B)_{b}^{\prime}$ continuously, as well. 
(4) $\mathcal{V}$ is regularly decreasing if, and only if, $\Lambda(B)$ is quasi-normable (and this is equivalent to $\Lambda(B)_{b}^{\prime}$ satisfying the strict Mackey convergence condition).

Proof. From Corollary 2.7, we know that $\mathfrak{V}$ is regularly decreasing if, and only if, $\checkmark C(\mathbf{N})$ is boundedly retractive; i.e., if, and only if, $\mathcal{}(\mathbf{N})$ satisfies the strict Mackey convergence condition. Next, note that $\mathcal{T}$ being regularly decreasing implies $\Lambda(B)_{b}^{\prime}$ $=\mathscr{V} C(\mathbf{N})$ topologically. In fact, if $\mathscr{T}$ satisfies condition $(\mathrm{wV})$, the full force of $[\mathbf{7}$, Appendix] shows that each bounded subset of $C \bar{V}(\mathbf{N})=\Lambda(B)_{b}^{\prime}$ is metrizable whereby $\Lambda(B)_{b}^{\prime}$ is bornological (e.g., see Köthe [23, I, §29, 4. (3) and the subsequent remark]). Hence, we can conclude that $\mathcal{V} C(\mathbf{N})=\Lambda(B)_{b}^{\prime}$ since $\mathcal{V} C(\mathbf{N})$ is the bornological space associated with $\Lambda(B)_{b}^{\prime}$. At this point, it remains to note that an infrabarrelled 1.c. space $E$ is quasi-normable if, and only if, its strong dual $E_{b}^{\prime}$ satisfies the strict Mackey convergence condition (Grothendieck [15, p. 106]) and that a metrizable quasi-normable space is distinguished [15, Proposition 14].

As a corollary to (4), we obtain:

(5) If $\mathcal{V}$ is regularly decreasing, $\Lambda(B)$ is distinguished, $C \bar{V}(\mathbf{N})=\Lambda(B)_{b}^{\prime}=\mathscr{V} C(\mathbf{N})$ topologically, and $\widetilde{V} C(\mathbf{N})$ is the strong bidual of both $\Upsilon_{0} C(\mathbf{N})$ and $C \bar{V}_{0}(\mathbf{N})$.

However, none of the implications in (5) can be reversed. Indeed, if $\Lambda(B)$ is an $(F M)$-space, which is not a Schwartz space (e.g., see Köthe [23, I, §31, 5.]), then $\Lambda(B)$ is distinguished, but not quasi-normable. Hence, in this case, $\mathscr{V}$ is not regularly decreasing, $\mathcal{V}_{0} C(\mathbf{N})$ is not complete, not semireflexive, and not closed in $\mathscr{V} C(\mathbf{N})$-in fact, $\widehat{\mathcal{V}_{0} C(\mathbf{N})}=C \bar{V}_{0}(\mathbf{N})=C \bar{V}(\mathbf{N})=\mathcal{V} C(\mathbf{N})$, but $\Lambda(B)_{b}^{\prime}=\mathcal{V} C(\mathbf{N})$ and $\left(\mathcal{V}_{0} C(\mathbf{N})_{b}^{\prime}\right)_{b}^{\prime}=$ $\left(C \bar{V}_{0}(\mathbf{N})_{b}^{\prime}\right)_{b}^{\prime}=\mathcal{V} C(\mathbf{N})$ topologically.

5. Concluding remarks. The local compactness hypothesis on $X$ was used in an essential way to prove the general results in the first part of $\S 1$. However, it is possible to derive an analogue of Theorem 1.6 when $X$ is a completely regular hemicompact Hausdorff space, and we sketch the proof of such a result in the first part of this section. In particular, this allows for the case $A(X)=H(X)$ where $X$ is an open subset of an infinite dimensional (DFM)-space $F$. In the second part of this section, we turn to regularity properties of $\mathscr{D} \mathcal{V} C(X, E)=\operatorname{ind}_{n \rightarrow} C V_{n}\left(X, E_{n}\right)$, where $E=$ ind $_{n \rightarrow} E_{n}$ is an inductive limit of certain type, and are thereby able to note a more general result than the one established prior to 1.12 (also see part (i) of the direct proof of Theorem 1.3(d)).

Let $X$ denote an arbitrary nonempty set. Endowed with the discrete topology, $X$ is a locally compact Hausdorff space, and any nonnegative real valued function on $X$ is a weight. For a system $V$ of weights on $X$ as in 0.1 (which, by definition, will always satisfy $\mathscr{K} \leqslant V$ when $X$ has the discrete topology) and a locally convex (Hausdorff) space $E$, we define

$$
\begin{array}{r}
F V(X, E)=\left\{f: X \rightarrow E ; q_{v, p}(f)=\sup \{v(x) p(f(x)) ; x \in X\}<\infty\right. \\
\text { for all } v \in V \text { and all } p \in c s(E)\} .
\end{array}
$$

Of course, assuming that $X$ has the discrete topology, $F V(X, E)$ coincides with $C V(X, E)$ as defined in 0.1 and is complete (under its natural 1.c. topology) whenever $E$ is complete. Correspondingly, for a decreasing sequence $\mathcal{V}=\left\{V_{n}\right\}_{n \in \mathrm{N}}$ of 
systems of weights on $X$, put $\mathfrak{V} F(X, E)=$ ind $_{n \rightarrow} F V_{n}(X, E)$, the space which we called $\widetilde{ } C(X, E)$ in 0.2 . Finally, we let $\overline{\bar{V}}$ denote the maximal system $\bar{V}_{\mho}$ associated with $\mathfrak{V}$. All other notation, like $\mathfrak{V} A(X, E)$ for some specified linear subspace $A(X, E)$ of the space $F(X, E)$ of all functions from $X$ into $E$, remains unchanged.

5.1. Proposition. Let $X$ be a nonempty set, $E$ a normed space, and $\mathfrak{V}=\left\{v_{n}\right\}_{n \in \mathrm{N}}$ be a decreasing sequence of weights on $X$. Further, we let $\delta=\left\{S_{k}\right\}_{k \in \mathbf{N}}$ denote an increasing sequence of nonempty subsets of $X$ with $X=\cup\left\{S_{k} ; k \in \mathbf{N}\right\}$, and assume that, for any pair $(n, k) \in \mathbf{N} \times \mathbf{N}$, there exist constants $c_{n k}, C_{n k}>0$ such that $c_{n k} \leqslant\left. v_{n}\right|_{S_{k}} \leqslant C_{n k}$. If

$\left(\mathrm{V}_{\S}\right)$ given $n \in \mathbf{N}$, there exists $m>n$ such that for every $\varepsilon>0$ it is possible to find

$$
k=k(\varepsilon) \in \mathbf{N} \text { with } v_{m}(x) / v_{n}(x)<\varepsilon \text { for all } x \in X \backslash S_{k},
$$

then $\mathfrak{V} F(X, E)=F \overline{\bar{V}}(X, E)$ algebraically and topologically.

An obvious modification of the direct proof of Theorem 1.3(d) can be used to establish Proposition 5.1. The assumption that $X$ is $\sigma$-compact in part (i) of that argument corresponds to $\delta=\left\{S_{n}\right\}_{n}$ being countable, while condition (V) is replaced by $\left(V_{\S}\right)$, which again is only used to show that the two topologies agree. Note also that the role of the partitions of unity here gives way to a simple cut-off procedure so that, in fact, the argument becomes much simpler; we omit the details.

By the way, condition $\left(\mathrm{V}_{\S}\right)$, like $(\mathrm{V})$, also implies $(\mathrm{wV})($ see $2.2(\mathrm{c}))$, and hence $\mathcal{V}=\left\{v_{n}\right\}_{n}$ in 5.1 is regularly decreasing. However, 5.1 does not follow from Theorem 2.3.

5.2. Corollary. Let $X$ be a locally compact Hasudorff space, $E$ be a normed space, and take $\mathfrak{V}=\left\{v_{n}\right\}_{n \in \mathrm{N}}$ to be a decreasing sequence of (u.s.c.) weights on $X$ such that $\inf \left\{v_{n}(x) ; x \in K\right\}>0$ for each compact subset $K$ of $X, n=1,2, \ldots$ If $\mathfrak{V}$ satisfies condition $(\mathrm{V})$, then $\mathfrak{V} C(X, E)$ is a topological subspace of $\mathfrak{V} F(X, E)$.

Proof. From 1.3(d) and its refinement in 1.11 , we know that $\mathfrak{} C(X, E)=$ $\Upsilon_{0} C(X, E)$ coincides with $C \bar{V}_{0}(X, E)=C \bar{V}(X, E)$ (algebraically and) topologically. On the other hand, since $X$ is $\sigma$-compact by 0.3 , and letting $\delta=\left\{S_{n}\right\}_{n \in N}$ denote a countable increasing basis for the compact subsets of $X$, condition $\left(\mathrm{V}_{\delta}\right)$ is just $(\mathrm{V})$. Hence we may apply 5.1 to obtain $\checkmark F(X, E)=F \overline{\bar{V}}(X, E)$ (algebraically and) topologically. Now, $\bar{V} \subset \overline{\bar{V}}$, while $\overline{\bar{V}} \leqslant \bar{V}$ since every $v_{n}$ is u.s.c. (Of course, all functions in $\overline{\bar{V}}$ need not be u.s.c. on $X$.) Thus, $C \bar{V}(X, E)$ is a topological linear subspace of $F \overline{\bar{V}}(X, E)$ which finishes the proof.

Quite obviously, Proposition 5.1 may be applied to situations other than the one in 5.2. There are, for instance, interesting consequences for sequence spaces on $\mathbf{N} \times \mathbf{N}$, say, or when $X$ is a normed linear space and $\delta$ is a countable increasing basis for the bounded subsets of $X$. Finally, there is the application alluded to earlier where $X$ is a completely regular hemicompact Hausdorff space and $\delta$ is a countable increasing basis for the compact sets in $X$, in which case condition $\left(\mathrm{V}_{\S}\right)$ again turns into (V). In this last situation, an analogue of 1.6 is available. 
5.3. Proposition. Let $X$ denote a completely regular hemicompact Hausdorff space, take $\mathfrak{V}=\left\{v_{n}\right\}$ to be a decreasing sequence of nonnegative functions $v_{n}$ on $X$ such that $v_{n}$ is both bounded and bounded away from 0 on each compact subset of $X, n=1,2, \ldots$, and let $A(X)$ denote a linear space of (complex valued) functions on $X$ which are bounded on all compact sets in $X$. If we assume that $\mathfrak{V}$ satisfies condition $(\mathrm{V})$ and that $A(X)$ is a semi-Montel space under the compact-open topology co, then $\mathfrak{V} A(X)=$ $\mathcal{V}_{0} A(X)$ is a Silva space which equals $A \overline{\bar{V}}(X)=A \overline{\bar{V}}_{0}(X)$ algebraically and topologically. Moreover, if each $v_{n}$ is u.s.c., then $\overline{\bar{V}}$ can be replaced by $\bar{V}$.

SKETCH OF PROOF. By our preceding remarks, if $\mathcal{S}$ denotes a countable increasing basis for the compact subsets of $X$, then 5.1 yields $\mathscr{V} F(X)=F \overline{\bar{V}}(X)$ algebraically and topologically. To show that $\mathfrak{V} A(X)$ is a Silva space, given $n \in \mathbf{N}$, let $m>n$ be chosen as in condition (V). Then compactness of the canonical injection $A v_{n}(X) \rightarrow$ $A v_{m}(X)$ follows since $(A(X), c o)$ is a semi-Montel space and from the easily established fact $[7,1.6]$ that, because of $(\mathrm{V}), A v_{m}(X)$ induces the compact-open topology on each bounded subset of $A v_{n}(X)$. Next, the inductive limit $\mathfrak{V} F(X)=$ ind $_{n \rightarrow} F v_{n}(X)$ is regular. (See, e.g., [7, 1.7.]. Or, a slight refinement of the argument leading to the algebraic equality $\mathscr{V} F(X)=F \overline{\bar{V}}(X)$ can be used to show that, for each bounded subset $B$ of $F \overline{\bar{V}}(X)$, there exists $n \in \mathbf{N}$ such that $B$ is bounded in $F v_{n}(X)$.) At this point, an application of Baernstein's open mapping lemma [1] yields that $\mathfrak{V} A(X)$ is a topological linear subspace of $\mathscr{V} F(X)$ whereby $\mathscr{V} A(X)=$ $A \overline{\bar{V}}(X)$ algebraically and topologically. Finally, the equations $\mathfrak{V} A(X) \equiv \Upsilon_{0} A(X)$ and $A \overline{\bar{V}}(X)=A \overline{\bar{V}}_{0}(X)$ are easily derived from (V) (the functions in $\overline{\bar{V}}$ are also bounded on compact sets in $X$ ), while the last assertion follows as in the proof of 5.2 .

5.4. CoRollary. Let $X$ be an open subset of a (DFM)-space $F$, and let $\mathcal{V}=\left\{v_{n}\right\}_{n \in \mathbf{N}}$ be a decreasing sequence of weights on $X$ such that $\inf \left\{v_{n}(x) ; x \in K\right\}>0$ for each compact subset $K$ of $X, n=1,2, \ldots$ If $\mathscr{T}$ satisfies condition $(\mathrm{V})$, then

$$
\mathfrak{V} H(X)=\mathfrak{V}_{0} H(X)=H \bar{V}_{0}(X)=H \bar{V}(X)
$$

algebraically and topologically and $\mathfrak{V} H(X)$ is a Silva space.

Proof. Open subsets of (DFM)-spaces are hemicompact $k$-spaces, and the space $(H(X), c o)$ of all holomorphic functions on $X$, endowed with the compact-open topology, is a Fréchet-Montel space (e.g., see [9]). So 5.4 follows readily from 5.3.

5.5. EXAmple. Let $X$ be an open subset of a (DFM)-space $F$, and let $\left(K_{n}\right)_{n \in \mathrm{N}}$ denote a countable increasing basis for the compact sets in $X$. If $\left(a_{n}\right)_{n \in \mathrm{N}}$ denotes a sequence of positive numbers with $\sum_{n=1}^{\infty} a_{n} \leqslant 1$, then $v=\sum_{n=1}^{\infty} a_{n} 1_{K_{n}}$ is a weight on $X$ which is bounded (by 1), bounded away from 0 on each compact subset of $X$, and which vanishes at infinity. Hence, putting $v_{n}=v^{n}, n=1,2, \ldots, \mathcal{V}=\left\{v_{n}\right\}_{n \in \mathrm{N}}$ is a system of weights on $X$ which satisfies all the hypotheses of 5.4. Note that, if $f$ is any holomorphic function on $X$, we may take $a_{n}=\left[2^{n} \max \left(1, \sup \left\{|f(x)| ; x \in K_{n}\right\}\right)\right]^{-1}$, $n=1,2, \ldots$, in the above construction so that $f \in H v_{1}(X) \subset \mathcal{V} H(X)$. 
The second part of this section is devoted to a sharpened vector valued form of a result originally due to B. A. Taylor [37] (see [7, 2.8]), and we will assume the following general setting. We will let $X$ denote a locally compact Hausdorff space, take $\mathscr{V}=\left\{v_{n}\right\}_{n \in \mathrm{N}}$ to be a decreasing sequence of weights $v_{n}$ on $X$ such that $\inf \left\{v_{n}(x) ; x \in K\right\}>0$ for each compact subset $K$ of $X, n=1,2, \ldots$, and let $E$ denote the 1.c. inductive limit ind ${ }_{n \rightarrow} E_{n}$ of an injective inductive sequence of 1.c. (Hausdorff) spaces. Since the proofs of the following results are not too difficult, only sketches will be provided.

5.6. LEMMA. If $E=\operatorname{ind}_{n \rightarrow} E_{n}$ is regular, then, for each bounded subset $B$ of $C \bar{V}(X, E)$, there exists $n \in \mathbf{N}$ such that $B(X)=\{f(x) ; f \in B, x \in X\}$ is contained in $E_{n}$. Moreover, if all the weights $v_{n}$ are continuous, $C \bar{V}(X, E)$ may be replaced by $C \tilde{V}(X, E)$.

5.7. LEMma. If $E=\operatorname{ind}_{n \rightarrow} E_{n}$ is a regular inductive limit of normed spaces $E_{n}$, then, for each bounded subset $B$ of $C \bar{V}(X, E)$, there exists $m \in \mathbf{N}$ such that $\left(v_{m} B\right)(X)=$ $\left\{v_{m}(x) f(x) ; f \in B, x \in X\right\}$ is bounded in $E_{m}$. Again, if all weights $v_{n}$ are continuous, $C \tilde{V}(X, E)$ can be used instead of $C \bar{V}(X, E)$.

The proof of 5.6 being similar (and even easier), we sketch only the proof of 5.7. Now, for arbitrary $\bar{v} \in \bar{V}$, the set $(\bar{v} B)(X)=\{\bar{v}(x) f(x) ; f \in B, x \in X\}$ is bounded in $E$, and so, by regularity of $E=$ ind $_{n \rightarrow} E_{n}$, is a bounded subset of $E_{n}$ for some $n=n(\bar{v}) \in \mathbf{N}$. Since $\mathscr{K} \leqslant \bar{V}$ holds because of our assumption on the weights $v_{n}$, we can conclude that, for each compact set $K$ in $X$, there exists $n=n(K)$ such that $B(K)=\{f(x) ; f \in B, x \in K\}$ is a bounded subset of $E_{n}$.

From 5.6, we infer that $B(X) \subset E_{n_{0}}$ for some $n_{0} \in \mathbf{N}$, and hence $\left(v_{m} B\right)(X) \subset E_{n}$ for all $m \in \mathbf{N}$ and all $n \geqslant n_{0}$. Now suppose that $\left(v_{m} B\right)(X)$ is unbounded in $E_{n}$ for each $m \in \mathbf{N}$ and each $n \geqslant n_{0}$. By induction, it follows that there exist a strictly increasing sequence $\left(k_{m}\right)_{m}$ of positive integers, $\left(f_{m}\right)_{m} \subset B,\left(x_{m}\right)_{m} \subset X$, and a sequence $\left(U_{m}\right)_{m}$ of open and relatively compact neighborhoods $U_{m}$ of $x_{m}$ such that $U_{m} \subset X \backslash\left(\cup_{k=1}^{m-1} \overline{U_{k}}\right)$ and $v_{m}\left(x_{m}\right)\left\|f_{m}\left(x_{m}\right)\right\|_{k_{m}} \geqslant m^{3}$. At this point, we choose $\varphi_{n} \in$ $C(X), 0 \leqslant \varphi_{n} \leqslant 1$, with $\varphi_{n}\left(x_{n}\right)=1$, but so that $\varphi_{n} \equiv 0$ on $X \backslash U_{n}, n=1,2, \ldots$ The function $\bar{v}=\sum_{n=1}^{\infty} \varphi_{n} v_{n} / n^{2}$ belongs to $\bar{V}$ (resp., $\tilde{V}$ whenever all weights $v_{n}$ are continuous) and satisfies $\bar{v}\left(x_{m}\right) \geqslant v_{m}\left(x_{m}\right) / m^{2}, m=1,2, \ldots$ Hence

$$
\bar{v}\left(x_{m}\right)\left\|f_{m}\left(x_{m}\right)\right\|_{k_{m}} \geqslant \frac{1}{m^{2}} v_{m}\left(x_{m}\right)\left\|f_{m}\left(x_{m}\right)\right\|_{k_{m}} \geqslant m \text { for } m=1,2, \ldots,
$$

which implies that $(\bar{v} B)(X)$ is unbounded in $E_{n}, n=1,2, \ldots$ This, however, is a contradiction to the remark at the beginning of the proof, and so the desired conclusion follows.

For the moment, let us suppose that $X$ is a nonempty set and endow $X$ with the discrete topology. In the notation introduced at the beginning of this section, Lemma 5.7, when applied to this situation, yields the following observation.

Let $\mathscr{V}=\left\{v_{n}\right\}_{n \in \mathbf{N}}$ be a decreasing sequence of positive functions on $X$, and let $E$ denote the 1.c. inductive limit of a regular injective inductive sequence of normed spaces $E_{n}$. Then a subset $B$ of $F \overline{\bar{V}}(X, E)$ is bounded if, and only if, there exists 
$n=n(B) \in \mathrm{N}$ such that $\left(v_{n} B\right)(X)$ is bounded in $E_{n}$, which clearly gives us that ind $_{n \rightarrow} F v_{n}\left(X, E_{n}\right)=\mathfrak{V} F(X, E)=F \overline{\bar{V}}(X, E)$ algebraically, that these three spaces have the same bounded subsets, and that $\operatorname{ind}_{n \rightarrow} F v_{n}\left(X, E_{n}\right)$ is a regular inductive limit.

To get the same result for continuous functions on arbitrary locally compact spaces, however, we need a stronger hypothesis on $E$.

5.8. Proposition. Let $X$ denote a locally compact Hausdorff space, and let $\mathfrak{V}=$ $\left\{v_{n}\right\}_{n \in \mathrm{N}}$ be a decreasing sequence of weights $v_{n}$ on $X$ such that $\inf \left\{v_{n}(x) ; x \in K\right\}>0$ for each compact subset $K$ of $X, n=1,2, \ldots$ If $E=\operatorname{ind}_{n \rightarrow} E_{n}$ is a boundedly retractive (or, equivalently, compactly regular; see the result of Neus [25] recalled in §0.3) injective inductive limit of normed spaces, then

(a) $\mathbb{D} \mathcal{V} C(X, E)=\widetilde{V} C(X, E)=C \bar{V}(X, E)$ algebraically, the three spaces have the same bounded sets, and both ind ${ }_{n \rightarrow} C v_{n}\left(X, E_{n}\right)$ and ind $_{n \rightarrow} C v_{n}(X, E)$ are regular;

(b) $\mathbb{D} \mathfrak{V} C(X, E)$ is the bornological space associated with $C \bar{V}(X, E)$ (or with $\checkmark C(X, E))$, and also the ultrabornological space associated with $C \bar{V}(X, E)$ if $E$ is complete ( which would follow if $E_{n}$ is complete for each $n=1,2, \ldots$ ).

Further, if all the weights $v_{n}$ are continuous, $\bar{V}$ may be replaced by $\tilde{V}$ in (a) and (b).

SKETCH OF PROOF. (a) In view of 5.7, for a given bounded set $B \subset C \bar{V}(X, E)$ (resp. $C \tilde{V}(X, E)$ ), there exists $n \in \mathbf{N}$ such that $\left(v_{n} B\right)(X)$ is bounded in $E_{n}$. $E=$ ind $_{k \rightarrow} E_{k}$, being a boundedly retractive inductive limit of normed spaces, is clearly strongly boundedly retractive; let $m \geqslant n$ be chosen so that the topologies of $E$ and $E_{m}$ coincide on each bounded subset of $E_{n}$. For $B$ to be bounded in $C v_{n}\left(X, E_{m}\right)$, and hence in $C v_{m}\left(X, E_{m}\right)$, it suffices to show that each $f \in B$ is a continuous mapping from $X$ into $E_{m}$. However, since $X$ is locally compact, it is enough to prove $\left.f\right|_{K}: K \rightarrow E_{m}$ is continuous for each compact subset $K$ of $X$. But this is now straightforward, as is the rest of the argument.

(b) $\mathscr{D} \mathcal{V} C(X, E)$, being an inductive limit of normed spaces, is bornological; the first assertion therefore follows from (a) and a well-known property of associated bornological topologies (e.g., see Horváth [21, 3, §7, Exercise 8]). If $E$ is complete, then $C \bar{V}(X, E)$ and $C \tilde{V}(X, E)$ are also complete so that the associated bornological and ultrabornological spaces coincide.

Even if we take $E=\mathrm{C}$ in 5.8, the topologies of $\widetilde{V} C(X)$ and $C \bar{V}(X)$ can be different, as Example 1.12 shows. Also note that, in addition to the properties listed in 1.12 , we have that $\mathfrak{V}_{0} C(X) \neq C \bar{V}_{0}(X)$ algebraically by applying $2.4(\mathrm{c})$ and 2.5 , and $\mathfrak{V} C(X)=\operatorname{ind}_{n \rightarrow} C v_{n}(X)$ is not boundedly retractive by $2.4(\mathrm{c})$ and 2.7 .

If, however, we add the hypothesis that $\mathfrak{T}$ is regularly decreasing (or, equivalently, satisfies condition (wV); see 2.2(a)) in 5.8(a), we can establish a stronger regularity property for $\mathscr{Q} \mathcal{V} C(X, E)$ and $\mathfrak{V} C(X, E)$.

5.9. Proposition. In addition to the hypotheses of 5.8, assume that the sequence $\mathfrak{V}=\left\{v_{n}\right\}_{n \in \mathrm{N}}$ of weights is regularly decreasing.

(a) For any $n \in \mathbf{N}$, there exists $k>n$ such that $C_{k}\left(X, E_{k}\right)$ and $C \bar{V}(X, E)$ induce the same topology on each bounded subset of $C v_{n}\left(X, E_{n}\right)$. 
(b) ind ${ }_{n \rightarrow} C v_{n}\left(X, E_{n}\right)$ and ind ${ }_{n \rightarrow} C v_{n}(X, E)$ are boundedly retractive, whence, in particular, $\mathscr{Q} \mathcal{V} C(X, E)$ is complete (and $\mathscr{V} C(X, E)$ is quasi-complete) whenever $E_{n}$ is complete, $n=1,2, \ldots$.

ProOF. (SKETCH). (a) Without loss of generality, we may assume $\|\cdot\|_{m \mid E_{n}} \leqslant\|\cdot\|_{n}$ for each $m \geqslant n$. Fix $n \in \mathbf{N}$ and choose $k \geqslant n$ with the following two properties:

(i) the topologies of $E$ and $E_{k}$ coincide on each bounded subset of $E_{n}$;

(ii) for every $\varepsilon>0$, there is some $\bar{v} \in \bar{V}$ such that $\bar{v}(x)<v_{k}(x)$ implies $v_{k}(x) \leqslant$ $\varepsilon v_{n}(x)$. (This is possible since $E=$ ind $_{n \rightarrow} E_{n}$ is strongly boundedly retractive and $\mathcal{V}$ satisfies (wV).) Now, fix a bounded subset $B \subset C v_{n}\left(X, E_{n}\right)$ and take $M=M(B)>$ $\sup \left\{v_{n}(x)\|f(x)\|_{n} ; f \in B, x \in X\right\}$. For $\varepsilon>0$ and $f_{0} \in B$, choose $\bar{v}=\bar{v}(B) \in \bar{V}$ according to (ii) with $\varepsilon / 2 M$ used instead of $\varepsilon$. We note that, because of (i), there exists $p \in \operatorname{cs}(E)$ such that $e \in E_{n},\|e\|_{n} \leqslant 2 M$, and $p(e) \leqslant 1$ imply $\|e\|_{k} \leqslant \varepsilon$. At this point, one easily verifies that

$$
\begin{aligned}
&\left\{f \in B ; \sup _{x \in X} \bar{v}(x)\right.\left.p\left(f(x)-f_{0}(x)\right) \leqslant 1\right\} \\
& \subset\left\{f \in B ; \sup _{x \in X} v_{k}(x)\left\|f(x)-f_{0}(x)\right\|_{k} \leqslant \varepsilon\right\},
\end{aligned}
$$

from which the desired conclusion follows.

Combining (a) with 5.8(a) now yields (b).

We remark that 5.8 and 5.9 are still of interest even when $E$ is a normed space, or if we take $\mathcal{V}=\left\{v_{n}\right\}_{n}$ with $v_{n}=v$ for $n=1,2, \ldots$. Also, we note that these results cannot be obtained by tensor product methods since, in general, $C v_{n}(X) \varepsilon E_{n}$ is a proper subspace of $C v_{n}\left(X, E_{n}\right)$ (see, e.g., [5, II, Theorem 11]).

The following, however, is an abstract result in the $\varepsilon$-product setting which, in part, is parallel to 5.8 and 5.9 .

5.10. Proposition. Let both $E=\operatorname{ind}_{n \rightarrow} E_{n}$ and $F=$ ind $_{n \rightarrow} F_{n}$ denote boundedly retractive (or, equivalently, compactly regular) inductive limits of sequences of Banach spaces.

(a) Then ind $\ln _{n \rightarrow}\left(E_{n} \varepsilon F_{n}\right)=\operatorname{ind}_{n \rightarrow}\left(E_{n} \varepsilon F\right)=E \varepsilon F$ algebraically, and the three spaces have the same bounded subsets. Hence ind ${ }_{n \rightarrow}\left(E_{n} \varepsilon F_{n}\right)$ is the (ultra-) bornological space associated with $E \varepsilon F$ (or with ind ${ }_{n \rightarrow}\left(E_{n} \varepsilon F\right)$ ).

(b) For a given $n \varepsilon \mathbf{N}$, there exists $k>n$ such that $E_{k} \varepsilon F_{k}$ and $E \varepsilon F$ induce the same topology on each bounded subset of $E_{n} \varepsilon F_{n}$. Similarly, for any $n \varepsilon \mathbf{N}$, there exists $m>n$ such that $E_{m} \varepsilon F$ and $E \varepsilon F$ induce the same topology on each bounded subset of $E_{n} \varepsilon F$. Hence ind ${ }_{n \rightarrow}\left(E_{n} \varepsilon F_{n}\right)$ and ind $_{n \rightarrow}\left(E_{n} \varepsilon F\right)$ are strongly boundedly retractive which, in particular, yields that ind ${ }_{n \rightarrow}\left(E_{n} \varepsilon F_{n}\right)$ is complete and $\operatorname{ind}_{n \rightarrow}\left(E_{n} \varepsilon F\right)$ is quasi-complete.

SKETCH OF PROOF. (a) has already been noted in [7, 4.6]; the algebraic isomorphism follows from certain factorization theorems for continuous linear mappings with values in an inductive limit (also see [6]), and the rest of the assertion is then an easy consequence of Grothendieck's open mapping theorem (cf. [16, Théorème B]).

For (b), it is convenient to prove the second assertion first; it holds for an arbitrary 1.c. space $F$. So, let $E_{n}^{1}$ denote the closed unit ball of $E_{n}, n=1,2, \ldots$, and note that, 
for any $n \in \mathbf{N}$, there exists $m>n$ such that $E_{m}$ and $E$ induce the same topology on each bounded subset of $E_{n}$. Next, fix a bounded set $B \subset E_{n} \varepsilon F_{n}, u_{0} \in B$, and $\varepsilon>0$, and let $V$ denote an arbitrary neighborhood of 0 in $F$. Then $B\left(V^{0}\right)=\left\{u\left(f^{\prime}\right) ; u \in B\right.$, $\left.f^{\prime} \in V^{0}\right\}$ is bounded in $E_{n}$; let

$$
M=M(B)>\sup \left\{\left\|u\left(f^{\prime}\right)\right\|_{n} ; u \in B, f^{\prime} \in V^{0}\right\} .
$$

The topologies of $E$ and $E_{m}$ coincide on $2 M E_{n}^{1}$, and hence there exists a neighborhood $U=U(B)$ of 0 in $E$ such that $U \cap\left(2 M E_{n}^{1}\right) \subset \varepsilon E_{m}^{1}$. Now, clearly

$$
\left\{u \in B ;\left(u-u_{0}\right)\left(V^{0}\right) \subset U\right\} \subset\left\{u \in B ;\left(u-u_{0}\right)\left(V^{0}\right) \subset \varepsilon E_{m}^{1}\right\},
$$

from which we deduce that the topologies of $E_{m} \varepsilon F$ and $E \varepsilon F$ coincide on each bounded subset of $E_{n} \varepsilon F$.

The first part of 5.10(b) now follows by applying the second assertion twice, first to $\left(E_{n} \varepsilon F\right)_{n}$ and a given $n \varepsilon \mathbf{N}$, obtaining $m>n$, and then to $\left(F_{n} \varepsilon E_{m}\right)_{n}$ and the natural number $m$, and by observing that $E \varepsilon F \cong F \varepsilon E$ for arbitrary 1.c. spaces $E$ and $F$. The rest of the argument is obvious.

Proposition 5.10 applies, for example, to ind ${ }_{n \rightarrow}\left(C v_{n}(X) \varepsilon E_{n}\right)=$ ind $_{n \rightarrow} C\left(v_{n}\right)^{p}\left(X, E_{n}\right)$, ind ${ }_{n \rightarrow}\left(C v_{n}(X) \varepsilon E\right)=$ ind $_{n \rightarrow} C\left(v_{n}\right)^{p}(X, E)$ and $\mathcal{V} C(X) \varepsilon E$ whenever $\mathscr{V}=\left\{v_{n}\right\}_{n}$ is regularly decreasing and $E=$ ind $_{n \rightarrow} E_{n}$ is a boundedly retractive inductive limit of Banach spaces; we refer to [3] or [5] for the definition of $C V^{p}(X, E)$, and for the $\varepsilon$-product representation $C V(X) \varepsilon E=C V^{p}(X, E)$. Another application of 5.10 is formally noted below.

5.11. Corollary. Let $X$ denote a locally compact Hausdorff space, let $\mathscr{V}=\left\{v_{n}\right\}_{n}$ be a decreasing sequence of weights $v_{n}$ on $X$ such that $\inf \left\{v_{n}(x) ; x \in K\right\}>0$ for each compact subset $K$ of $X, n=1,2, \ldots$, and let $E$ be an injective inductive limit of $a$ sequence $\left(E_{n}\right)_{n}$ of Banach spaces.

If $\mathcal{W}$ is regularly decreasing and ind ${ }_{n \rightarrow} E_{n}$ is compactly regular, then

$$
\text { D } \mathcal{V}_{0} C(X, E)=\mathcal{V}_{0} C(X, E)=\mathcal{V}_{0} C(X) \varepsilon E=C \bar{V}_{0}(X, E)
$$

algebraically, while $\mathscr{Q} \mathscr{V}_{0} C(X, E)=\operatorname{ind}_{n \rightarrow} C\left(v_{n}\right)_{0}\left(X, E_{n}\right)$ and $\mathscr{V}_{0} C(X, E)=$ ind $_{n \rightarrow} C\left(v_{n}\right)_{0}(X, E)$ are strongly boundedly retractive.

Proof. Since ind ${ }_{n \rightarrow} C\left(v_{n}\right)_{0}(X)$ is boundedly retractive for regularly decreasing $\mathscr{V}$ as noted in the second proof of Theorem 2.3, we can apply 5.10, noting that $C\left(v_{n}\right)_{0}(X, E)=C\left(v_{n}\right)_{0}(X) \varepsilon E, n=1,2, \ldots$, and $C \bar{V}_{0}(X, E)=C \bar{V}_{0}(X) \varepsilon E$ for each complete l.c. space $E$.

Concerning the first conclusion of 5.11 , note that, under a slightly stronger hypothesis on $\mathfrak{V}=\left\{v_{n}\right\}_{n}$, Corollary 3.3. already gives $\mathcal{Q} \mathcal{V}_{0} C(X, E)=\mathcal{V}_{0} C(X, E)=$ $\mathfrak{V}_{0} C(X) \varepsilon E=C \bar{V}_{0}(X, E)$ topologically. For the corresponding spaces of functions belonging to a subspace $A(X, E)$ of $C(X, E)$, however, such a result is known to hold only under additional assumptions, but the techniques of this section can be put to good use in this direction.

5.12. Corollary. Let $X$ denote a locally compact Hausdorff space, and take $\mathfrak{V}=\left\{v_{n}\right\}_{n}$ to be a decreasing sequence of weights $v_{n}$ on $X$ such that $\inf \left\{v_{n}(x)\right.$; $x \in K\}>0$ for each compact subset $K \subset X, n=1,2, \ldots$ Further, let $E$ be $a$ 
boundedly retractive injective inductive limit of a sequence $\left(E_{n}\right)_{n}$ of normed spaces, and let $A(X, E)$ denote an arbitrary linear subspace of $C(X, E)$.

(a) $\mathscr{D} \mathcal{V} A(X, E)=\mathfrak{V} A(X, E)=A \bar{V}(X, E)$ holds algebraically, $\mathscr{D} \mathcal{V} A(X, E)$ is the bornological space associated with $A \bar{V}(X, E)$ (or with $\mathcal{T} A(X, E)$ ), and both ind $_{n \rightarrow} A v_{n}\left(X, E_{n}\right)$ and ind ${ }_{n \rightarrow} A v_{n}(X, E)$ are regular.

For the remaining conclusions, we also assume that $\mathscr{W}$ is regularly decreasing and that $A(X, E)$ is closed in $(C(X, E)$, co $)$.

(b) For any $n \in \mathbf{N}$, there exists $k>n$ such that $A v_{k}\left(X, E_{k}\right)$ and $A \bar{V}(X, E)$ induce the same topology on each bounded subset of $A v_{n}\left(X, E_{n}\right)$, both ind ${ }_{n \rightarrow} A v_{n}\left(X, E_{n}\right)$ and ind $_{n \rightarrow} A v_{n}(X, E)$ are boundedly retractive, and $\mathscr{Q} \mathcal{W} A(X, E)$ is complete (and $\mathcal{V} A(X, E)$ is quasi-complete) whenever $E_{n}$ is complete, $n=1,2, \ldots$

(c) Suppose that $E_{n}$ is a Banach space, $n=1,2, \ldots$, and that $A(X)$ is a closed linear subspace of $(C(X), \mathrm{co})$ with

$$
A(X, E)=\left\{f \in C(X, E) ; e^{\prime} \circ f \in A(X) \text { for all } e^{\prime} \in E^{\prime}\right\} .
$$

Then we have

$$
\text { D } \mathcal{V}_{0} A(X, E)=\mathfrak{V}_{0} A(X, E)=\mathfrak{V}_{0} A(X) \varepsilon E=A \bar{V}_{0}(X, E)
$$

algebraically, $\mathscr{D} \mathcal{V}_{0} A(X, E)$ is the (ultra-) bornological space associated with $\mathcal{V}_{0} A(X) \varepsilon E$ (or with $\mathfrak{V}_{0} A(X, E)$ ), both ind ${ }_{n \rightarrow} A\left(v_{n}\right)_{0}\left(X, E_{n}\right)$ and ind ${ }_{n \rightarrow} A\left(v_{n}\right)_{0}(X, E)$ are strongly boundedly retractive, and hence $\mathscr{Q} \mathfrak{V}_{0} A(X, E)$ is complete $\left(\mathfrak{V}_{0} A(X, E)\right.$ is quasi-complete).

SKETCH OF PROOF. Assertions (a) and (b) follow readily from 5.8 and 5.9, while (c) can be derived from 5.10 together with 5.11. $\left(A\left(v_{n}\right)_{0}(X, E)=A\left(v_{n}\right)_{0}(X) \varepsilon E, n=\right.$ $1,2, \ldots$, is again a consequence of [5, II, Theorem 29].)

\section{REFERENCES}

1. A. Baernstein II, Representation of holomorphic functions by boundary integrals, Trans. Amer. Math. Soc. 160 (1971), 27-37.

2. C. A. Berenstein and M. A. Dostal, Analytically uniform spaces and their applications to convolution equations, Lecture Notes in Math., vol. 256, Springer-Verlag, Berlin and New York, 1972.

3. K.-D. Bierstedt, Gewichtete Räume stetiger vektorwertiger Funktionen und das injektive Tensorproduct. I, J. Reine Angew. Math. 259 (1973), 186-210; II, J. Reine Angew. Math. 260 (1973), 133-146.

4. __ Injektive Tensorprodukte und Slice-Produkte gewichteter Räume stetiger Funktionen, J. Reine Angew. Math. 266 (1974), 121-131.

5. _ I. The approximation property for weighted function spaces, Bonner Math. Schriften 81 (1975), 3-25; II. Tensor products of weighted spaces, Bonner Math. Schriften 81 (1975), 26-58.

6. K.-D. Bierstedt and R. Meise, Bemerkungen über die Approximationseigenschaft lokalkonvexer Funktionenräume, Math. Ann. 209 (1974), 99-107.

7. Induktive Limites gewichteter Räume stetiger und holomorpher Funktionen, J. Reine Angew. Math. 282 (1976), 186-220.

8. K.-D. Bierstedt, B. Gramsch and R. Meise, Lokalkonvexe Garben und gewichtete induktive Limites F-morpher Funktionen, Bonner Math. Schriften 81 (1975), 59-72.

9. S. Dineen, Holomorphic functions on strong duals of Fréchet-Montel spaces, Infinite Dimensional Holomorphy and Applications, Math. Studies, no. 12, North-Holland, Amsterdam, 1977, pp. 147-166.

10. Holomorphic germs on compact subsets of locally convex spaces, Functional Analysis, Holomorphy and Approximation Theory, Lecture Notes in Math., vol. 843, Springer-Verlag, Berlin and New York, 1981, pp. 247-283.

11. L. Ehrenpreis, Fourier analysis in several complex variables, Interscience Tracts in Math., no. 17, Wiley, New York, 1970. 
12. J.-P. Ferrier, Spectral theory and complex analysis, Math. Studies, no. 4, North-Holland, Amsterdam, 1973.

13. K. Floret, Lokalkonvexe Sequenzen mit kompakten Abbildungen, J. Reine Angew. Math. 247 (1971), 155-195.

14. A. Goullet de Rugy, Espaces de fonctions pondérables, Israel J. Math. 12 (1972), 147-160.

15. A. Grothendieck, Sur les espaces $(F)$ et ( $D F$ ), Summa Brasil. Math. 3 (1954), 57-122.

16. __ Produits tensoriels topologiques et espaces nucléaires, Mem. Amer. Math. Soc. no. 16 (1955), reprint 1966.

17. O. v. Grudzinski, Convolutions-Gleichungen in Räumen von Beurling-Distributionen endlicher Ordnung, Habilitationsschrift, Kiel, 1980.

18. S. Hansen, On the "Fundamental Principle" of L. Ehrenpreis, Banach Center Publications (to appear).

19. L_ Localizable analytically uniform spaces and the Fundamental Principle, Trans. Amer. Math. Soc. 264 (1981), 235-250.

20. R. Hollstein, Inductive limits and $\varepsilon$-tensor products, J. Reine Angew. Math. 319 (1980), 38-62.

21. J. Horváth, Topological vector spaces and distributions. I, Addison-Wesley, Reading, Mass., 1966.

22. G. Kleinstück, Duals of weighted spaces of continuous functions, Bonner Math. Schriften 81 (1975), 98-114.

23. G. Köthe, Topological vector spaces. I, Grundlehren der Math. Wissenschaften, vol. 159; II, Grundlehren der Math. Wissenschaften, vol. 237, Springer-Verlag, Berlin and New York, 1969 and 1979.

24. R. Meise, Räume holomorpher Vektorfunktionen mit Wachstumsbedingungen und topologische Tensorprodukte, Math. Ann. 199 (1972), 293-312.

25. H. Neus, Über die Regularitätsbegriffe induktiver lokalkonvexer Sequenzen, Manuscripta Math. 25 (1978), 135-145.

26. B. E. Pedersen, Holomorphic functions with growth conditions, Trans. Amer. Math. Soc. 206 (1975), 395-406.

27. J. B. Prolla, The approximation property for Nachbin spaces, Approximation Theory and Functional Analysis, Math. Studies, no. 35, North-Holland, Amsterdam, 1979, pp. 371-382.

28. D. Raikov, Completeness criteria in locally convex spaces, Uspehi Mat. Nauk 14 (1959), 223-239. (Russian)

29. J. Schmets, Espaces de fonctions continues, Lecture Notes in Math., vol. 519, Springer-Verlag, Berlin and New York, 1976.

30. L. Schwartz, Espaces de fonctions différentiables à valeurs vectorielles, J. Analyse Math. 4 (1954), $88-148$.

31. __ Théorie des distributions à valeurs vectorielles. I, Ann. Inst. Fourier (Grenoble) 7 (1957), $1-142$.

32. C. Servien, Sur la topologie d'un espace de fonctions entières avec poids, Séminaire P. Lelong (Analyse), Année 1974/75, Lecture Notes in Math., vol. 524, Springer-Verlag, Berlin and New York, 1976, pp. 90-96; MR 54 \# 13546 (B. A. Taylor), Zbl. 349.46024 (K.-D. Bierstedt).

33. W. H. Summers, Weighted locally convex spaces of continuous functions, $\mathrm{Ph}$. D. Dissertation, Louisiana State University, Baton Rouge, 1968.

34. A A representation theorem for biequicontinuous completed tensor products of weighted spaces, Trans. Amer. Math. Soc. 146 (1969), 121-132.

35. __ Dual spaces of weighted spaces, Trans. Amer. Math. Soc. 151 (1970), 323-333.

36. B. A. Taylor, Some locally convex spaces of entire functions, Proc. Sympos. Pure Math., Entire Functions and Related Parts of Analysis, vol. 11, Amer. Math. Soc., Providence, R. I., 1968, pp. 431-467.

37. A seminorm topology for some (DF)-spaces of entire functions, Duke Math. J. 38 (1971), 379-385.

Universität-GH-Paderborn, FB 17, Mathematik, D2-228, Warburger Strasse 100, Postfach 1621, D-4790 PADERborn, Federal Republic OF Germany

UNIVERSITÄt DUSSELDORF, MATHEMATISCHES INSTITUT, UNIVERSITATSSTRASSE 1, D-4000 DUSSELDORF, FEDERAL REPUBLIC OF GERMANY

Department of Mathematics, University of ARKansas, Fayetteville, ArKansas 72701 